\title{
From double Lie groups to quantum groups
}

\author{
by \\ Piotr Stachura (Washington, DC, and Warszawa)
}

\begin{abstract}
It is shown, using geometric methods, that there is a $C^{*}$-algebraic quantum group related to any double Lie group (also known as a matched pair of Lie groups or a bicrossproduct Lie group). An algebra underlying this quantum group is the algebra of a differential groupoid naturally associated with the double Lie group.
\end{abstract}

0. Introduction. The theory of objects we now call Quantum Groups is from the very beginning related to group factorizations. First constructions are due to Kac [5]. Then important contributions, algebraic (double cross product of Hopf algebras) as well as functional-analytic (in the context of locally compact groups and Kac-von Neumann algebras) were made by Majid [7, 8]. (See also [9] for Lie group factorization and a detailed exposition of the algebraic structure.) The Kac algebras framework is covered in [4]. Afterwards, Baaj and Skandalis created their theory of multiplicative unitaries [1] and showed that such a multiplicative unitary is defined by any factorization of a locally compact group. From this moment on one could expect that there is a $C^{*}$-algebraic quantum group behind any (locally compact) group factorization. And indeed, before this work was finished, a very general construction of $C^{*}$-algebraic quantum groups related to even more general situations (cocycle matched pairs of locally compact quantum groups) had appeared [15].

This paper is devoted to a construction of $C^{*}$-quantum groups from factorizations known as double Lie groups (DLG) [6] and also as matched pairs of Lie groups. Although this situation is obviously less general than the one considered in [15], precisely for that reason we are able to use different, more geometric methods.

Objects used in our constructions are defined on a smooth level and we try to remain within the smooth category as long as possible. All of these

2000 Mathematics Subject Classification: 81R50, 22A22, 22E99.

Key words and phrases: double Lie group, quantum group.

The work was supported by Polish KBN grant No. 2 P03A 04022. 
objects have a clear geometric interpretation as objects related to differential groupoids naturally associated with DLGs. Starting from a concrete algebra of such objects we construct (at this smooth level) various mappings which appear in the theory of quantum groups. Then by using density arguments we lift those mappings to the $C^{*}$-algebraic level.

An additional advantage of the geometric approach we use in this paper is that, after recognizing the geometric content of a formula, we can usually prove it using only the very standard and well known results from calculus: the Fubini theorem and change of variables for the Riemann integral of smooth functions.

Although from a theoretical point of view this work adds nothing to the pure theory of quantum groups, it shows, we believe, interesting links between the theory of quantum groups and differential geometry. In the examples constructed this way we have a natural, dense $*$-subalgebra of "smooth" elements in our $C^{*}$-algebra, which, putting aside subtleties with densities, is an algebra of smooth, compactly supported functions, with a kind of convolution as product. As a result, we can use various techniques and estimates from the theory of integral and appeal to geometric intuition in order to perform our computations.

There are also connections of our work with "quantization" of some semidirect product Poisson-Lie groups, among them the $\kappa$-Poincaré group. However, in this case the situation is not so good. Namely, the construction presented in this paper does not directly apply, since the set of decomposable elements in $G$, i.e. $\{g \in G: g=a b=\widetilde{b} \widetilde{a}, a, \widetilde{a} \in A, b, \widetilde{b} \in B\}$ is only open and dense in $G$, instead of being equal to $G$. Nevertheless, the methodology we propose can be applied also in this case and may give correct results.

This paper is full of long integrals and other technical details. Therefore, in order to improve its readability, we sketch the conceptual framework, which, we believe, is simple and natural. For any DLG $(G ; A, B)$ there is a naturally defined multiplicative unitary operator (in fact there are four of them). This operator is manageable in the sense of Woronowicz as was proved in [12]. Therefore, by the results of Woronowicz from [16], there is a quantum group (modulo the problem of Haar measure) associated with any DLG.

Additionally, given a triple $(G ; A, B)$, one can define two differential groupoid structures on $G$ : $G_{A}$ and $G_{B}$ over $A$ and $B$, respectively. It turns out that the multiplication of one of the groupoids, say $G_{B}$, defines a morphism in the sense of Zakrzewski [17] from $G_{A}$ to $G_{A} \times G_{A}$. This morphism is coassociative. Using a construction presented in [13] we get a coassociative morphism from $C^{*}\left(G_{A}\right)$ to $C^{*}\left(G_{A} \times G_{A}\right)$. We may suppose that $C^{*}\left(G_{A} \times G_{A}\right)$ is (some sort of) $C^{*}\left(G_{A}\right) \otimes C^{*}\left(G_{A}\right)$. Therefore, the morphism constructed as 
above begins to resemble a comultiplication, one of the main ingredients of a quantum group structure. Completion of such construction of comultiplication is complicated by the fact that we are not able to prove the equality $C^{*}\left(G_{A} \times G_{A}\right)=C^{*}\left(G_{A}\right) \otimes C^{*}\left(G_{A}\right)$. Nevertheless, we have a corresponding morphism of reduced $C^{*}$-algebras. It turns out, as one can expect, that this morphism is implemented by the multiplicative unitary.

Due to great richness of the structure of a DLG, its other components enable us to find natural objects giving us almost a Hopf algebra structure. Moreover, they permit constructing an invariant positive functional on it with a natural modular group. To be more specific, the group inverse implements the unitary part of an antipode; a natural cocycle on the groupoid implements a scaling group; a distinguished fiber gives a "counit"; and Haar density on the set of units of the groupoid gives an invariant functional. We give explicit formulae for all of these mappings.

Let us now briefly describe the content of the paper. In Section 1 we collect basic facts about differential groupoids, their morphisms and related algebras, in order to make the paper more self-contained. Section 2 is devoted to proofs of some technical results used later on. In the third section we present groupoids related to DLG. In Section 4 we prove that our constructions indeed provide a comultiplication in the sense of the theory of quantum groups. In Section 5 we show that the algebra of a groupoid $G_{A}$ is very similar to a Hopf algebra, by identifying other ingredients of its structure and proving some formulae. The sixth section is devoted to the Haar measure. We use the results from [13], where it was shown that there is a natural class of KMS weights on any differential groupoid. Since in the groupoid $G_{A}$ the set of units is the group $A$, one can distinguish invariant half-densities on $A$. These half-densities give us a Haar measure on a quantum group based on $G_{A}$. In the last section we collect all the constructions and results. Finally, there are two appendices, where we give a geometric interpretation of a cocycle implementing a scaling group, and relate an algebra associated to a multiplicative unitary with a groupoid algebra.

To end this introduction, the author wants to record that the idea of the construction presented in this paper was born during his collaboration with Stanisław Zakrzewski. The author also wishes to thank Robert Owczarek for his useful comments.

1. Differential groupoids, their morphisms and algebras. In this section we introduce notation and recall basic facts about groupoids, their morphisms (in the sense of S. Zakrzewski) and action of morphisms on groupoid algebras. For a detailed exposition of the subject we refer to [17] (differential groupoids and morphisms) and [13] (constructions related to groupoid algebras). 
The category of differential groupoids. Let us recall that a differential relation $r$ from a manifold $X$ to a manifold $Y$ is a triple $(X, Y ; R)$ such that $R=: \operatorname{Gr}(r)$ is a submanifold in $Y \times X$. By a submanifold we always mean an embedded submanifold. All manifolds considered here are smooth, Hausdorff, and have countable bases of neighborhoods. A relation $r$ from $X$ to $Y$ will be denoted by $r: X \rightarrow Y$. For a relation $r: X \rightarrow Y$, we denote by $r^{T}$ the transposed relation, i.e. $r^{T}: Y \rightarrow X$ is given by $\operatorname{Gr}\left(r^{T}\right):=\{(x, y) \in X \times Y$ : $(y, x) \in \operatorname{Gr}(r)\}$.

A composition of differential relations may fail to be a differential relation. Therefore, in a definition below we assume that there are compositions which give again differential relations. There are also other conditions which, together with this composition property, are contained in the notion of transversality [17]. Transversality of differential relations $r$ and $s$ will be denoted by $r \nmid s$. A differential relation $r: X \rightarrow Y$ is a differential reduction if $r=f i^{T}$, where $i$ is the inclusion mapping of a submanifold $C \subset X$ and $f: C \rightarrow Y$ is a surjective submersion.

Now we recall the definition of a differential groupoid.

Definition 1.1 ([17]). Let $\Gamma$ be a manifold. A differential groupoid structure on $\Gamma$ is a triple $(m, s, e)$, where $m: \Gamma \times \Gamma \rightarrow \Gamma$ is a differential reduction, $e:\{1\} \rightarrow \Gamma$ is a differential relation, $s: \Gamma \rightarrow \Gamma$ is an involutive diffeomorphism and the following conditions are satisfied:

1. $m(m \times \mathrm{id})=m(\mathrm{id} \times m)$.

2. $m(e \times \mathrm{id})=m(\mathrm{id} \times e)=\mathrm{id}$.

3. $m(s \times s) \sim=s m$, where $\sim: \Gamma \times \Gamma \ni(x, y) \mapsto(y, x) \in \Gamma \times \Gamma$.

4. For any $x \in \Gamma, \emptyset \neq m(x, s(x)) \subset e(\{1\})$.

5. $m \uparrow(m \times \mathrm{id}), m \uparrow(\mathrm{id} \times m), m \uparrow(e \times \mathrm{id}), m \uparrow(\mathrm{id} \times e)$.

It follows that objects defined in this way coincide with standard differential groupoids. The set $E:=e(\{1\})$ is a submanifold of $\Gamma$ called the set of identities. There are also two projections (surjective submersions): the source or right projection $e_{R}: \Gamma \ni x \mapsto m(s(x), x) \in E$, and the target or left projection $e_{L}: \Gamma \ni x \mapsto m(x, s(x)) \in E$. The fiber of $e_{L}$ passing through $g$ (i.e. the set $\left\{x \in \Gamma: e_{L}(x)=e_{L}(g)\right\}$ ) will be denoted by $F_{l}(g)$, and similarly the fiber of $e_{R}$ by $F_{r}(g)$.

Now comes the definition of a morphism. It should be stressed that this definition does not coincide with the standard one. For examples and motivations see $[17,13]$.

Definition $1.2([17])$. Let $(\Gamma, m, s, e)$ and $\left(\Gamma^{\prime}, m^{\prime}, s^{\prime}, e^{\prime}\right)$ be differential groupoids. A differential relation $h: \Gamma \rightarrow \Gamma^{\prime}$ is a morphism from $\Gamma$ to $\Gamma^{\prime}$ iff

1. $h m=m^{\prime}(h \times h)$,

2. $h s=s^{\prime} h$, 
3. $h e=e^{\prime}$,

4. $m^{\prime} \uparrow(h \times h), h \uparrow e$.

In the next proposition we collect basic properties of objects associated with a morphism.

Proposition $1.3([17])$. Let $h: \Gamma \longrightarrow \Gamma^{\prime}$ be a morphism of differential groupoids.

1. The formula $\operatorname{Gr}\left(f_{h}\right):=\left(E \times E^{\prime}\right) \cap \operatorname{Gr}\left(h^{T}\right)$ defines a smooth mapping $f_{h}: E^{\prime} \rightarrow E$.

2. For each $b \in E^{\prime}$, the relation $h^{T}$ restricted to $F_{l}(b) \times F_{l}\left(f_{h}(b)\right)$ defines a smooth mapping $h_{b}^{L}: F_{l}\left(f_{h}(b)\right) \rightarrow F_{l}(b)$. The same is true for restriction to right fibers with the resulting map $h_{b}^{R}: F_{r}\left(f_{h}(b)\right) \rightarrow$ $F_{r}(b)$.

3. The set $\Gamma \times{ }_{h} \Gamma^{\prime}:=\left\{(x, y) \in \Gamma \times \Gamma^{\prime}: e_{R}(x)=f_{h}\left(e_{L}^{\prime}(y)\right)\right\}$ is a submanifold of $\Gamma \times \Gamma^{\prime}$.

4. The set $\Gamma *_{h} \Gamma^{\prime}:=\left\{(x, y) \in \Gamma \times \Gamma^{\prime}: e_{L}(x)=f_{h}\left(e_{L}^{\prime}(y)\right)\right\}$ is a submanifold of $\Gamma \times \Gamma^{\prime}$.

5. The sets $\Gamma *_{h} E^{\prime}$ and $\Gamma \times_{h} E^{\prime}$ are submanifolds and the mappings $h^{L}: \Gamma *_{h} E^{\prime} \ni(x, b) \mapsto h_{b}^{L}(x) \in \Gamma^{\prime}$ and $h^{R}: \Gamma \times_{h} E^{\prime} \ni(x, b) \mapsto$ $h_{b}^{R}(x) \in \Gamma^{\prime}$ are smooth.

6. The mapping $m_{h}: \Gamma \times_{h} \Gamma^{\prime} \ni(x, y) \mapsto m^{\prime}\left(h^{R}\left(x, e_{L}^{\prime}(y)\right), y\right) \in \Gamma^{\prime}$ is a surjective submersion.

7. The mapping $\mathfrak{t}_{h}: \Gamma \times_{h} \Gamma^{\prime} \ni(x, y) \mapsto\left(x, m_{h}(x, y)\right) \in \Gamma *_{h} \Gamma^{\prime}$ is a diffeomorphism.

8. The mapping: $\pi_{2}: \Gamma \times_{h} \Gamma^{\prime} \ni(x, y) \mapsto y \in \Gamma^{\prime}$ is a surjective submersion and $\pi_{2}^{-1}(y)$ is diffeomorphic to $F_{r}\left(f_{h}\left(e_{L}^{\prime}(y)\right)\right)$.

9. The mapping $\widetilde{\pi}_{2}: \Gamma *_{h} \Gamma^{\prime} \ni(x, y) \mapsto y \in \Gamma^{\prime}$ is a surjective submersion and $\tilde{\pi}_{2}^{-1}(y)$ is diffeomorphic to $F_{l}\left(f_{h}\left(e_{L}^{\prime}(y)\right)\right)$.

10. Items 6-9 remain true if we replace $\Gamma^{\prime}$ by $F_{r}(y)$ for any $y \in \Gamma^{\prime}$ and restrict the corresponding mappings in the obvious way.

Bidensities. Let $\Gamma$ be a differential groupoid and let $\Omega^{1 / 2}\left(e_{L}\right)$ (resp. $\left.\Omega^{1 / 2}\left(e_{R}\right)\right)$ be the bundle of smooth, complex-valued, half-densities along left (right) fibers of $\Gamma$. We denote by $\mathcal{A}(\Gamma)$ the linear space of compactly supported, smooth sections of the bundle $\Omega^{1 / 2}\left(e_{L}\right) \otimes \Omega^{1 / 2}\left(e_{R}\right)$ (see [2]). Its elements will be called bidensities and usually denoted by $\omega$. So $\omega(x)=\lambda(x) \otimes$ $\varrho(x) \in \Omega^{1 / 2} T_{x}^{l} \Gamma \otimes \Omega^{1 / 2} T_{x}^{r} \Gamma$, where $T_{x}^{l} \Gamma:=T_{x}\left(F_{l}(x)\right)$ and $T_{x}^{r} \Gamma:=T_{x}\left(F_{r}(x)\right)$. In the following we also write $\Omega_{L}^{1 / 2}(x):=\Omega^{1 / 2} T_{x}^{l} \Gamma$ and $\Omega_{R}^{1 / 2}(x):=\Omega^{1 / 2} T_{x}^{r} \Gamma$.

We also use the following notation: if $M, N$ are manifolds, $F: M \rightarrow N$ and $\psi$ is some geometric object on $M$ which can be pushed forward by $F$, then we denote the push forward of $\psi$ simply by $F \psi$. What it really means will be clear from the context. So below we write for example $s(v)$ instead 
of $\Lambda T_{x} s(v)$ for $v \in \Lambda^{\max } T_{x}^{l} \Gamma$ (for a vector space $V$ we denote by $\Lambda^{\max } V$ the maximal non-zero exterior power of $V$ ).

The groupoid inverse induces a star operation on $\mathcal{A}(\Gamma)$ :

$$
\omega^{*}(x)(v \otimes w):=\overline{\omega(s(x))(s(w) \otimes s(v))}, \quad v \in \Lambda^{\max } T_{x}^{l} \Gamma, w \in \Lambda^{\max } T_{x}^{r} \Gamma .
$$

Because $s$ is an involutive diffeomorphism which interchanges left and right fibers, the $*$-operation is a well defined antilinear involution.

One can define a multiplication on the vector space $\mathcal{A}(\Gamma)$. This multiplication introduces a $*$-algebra structure on $\mathcal{A}(\Gamma)$. The formula for multiplication is a special case of a more general construction presented in [13] and will be given later on. Before giving the formula, we define some special sections of $\Omega^{1 / 2}\left(e_{L}\right) \otimes \Omega^{1 / 2}\left(e_{R}\right)$, which are very convenient for computations.

Since left (right) translations are diffeomorphisms of left (right) fibers, we can define in a natural way left(right)-invariant sections of $\Omega^{1 / 2}\left(e_{L}\right)$ $\left(\Omega^{1 / 2}\left(e_{R}\right)\right)$. Any left-invariant section of $\Omega^{1 / 2}\left(e_{L}\right)$ is determined by its value on $E$ and, conversely, any section of $\left.\Omega^{1 / 2}\left(e_{L}\right)\right|_{E}$ can be uniquely extended to a left-invariant section of $\Omega^{1 / 2}\left(e_{L}\right)$.

So let $\widetilde{\lambda}$ be a non-vanishing, real, half-density on $E$ along left fibers (one constructs such a density by covering $E$ with maps adapted to the submersion $e_{L}$ and using an appropriate partition of unity to glue them together). We define

$$
\lambda_{0}(x)(v):=\widetilde{\lambda}\left(e_{R}(x)\right)(s(x) v), \quad v \in \Lambda^{\max } T_{x}^{l} \Gamma .
$$

This $\lambda_{0}$ is a left-invariant, real, non-vanishing section of $\Omega^{1 / 2}\left(e_{L}\right)$. Now, $\widetilde{\varrho}:=\widetilde{\lambda} s$ is a non-vanishing, real half-density on $E$ along right fibers, and $\varrho_{0}$ defined by

$$
\varrho_{0}(x)(w):=\widetilde{\varrho}\left(e_{L}(x)\right)(w s(x)), \quad w \in \Lambda^{\max } T_{x}^{r} \Gamma,
$$

is a right-invariant, non-vanishing, real section of $\Omega^{1 / 2}\left(e_{R}\right)$. Let $\omega_{0}:=\lambda_{0} \otimes \varrho_{0}$; this is a real, non-vanishing bidensity (of course, it does not belong to $\mathcal{A}(\Gamma)$ but we will still call it a bidensity). From now on the symbol $\omega_{0}$ will always mean a bidensity constructed in this way.

When $\omega_{0}$ is chosen any element $\omega \in \mathcal{A}(\Gamma)$ can be written uniquely as $\omega=f \omega_{0}$ for some smooth, complex-valued function $f$ with compact support. Note the following simple:

Lemma 1.4. If $\omega=f \omega_{0}$ then $\omega^{*}=f^{*} \omega_{0}$, where $f^{*}(x):=\overline{f(s(x))}$.

Action of groupoid morphisms on bidensities. It turns out that groupoid morphisms act on bidensities, i.e. for a morphism $h: \Gamma \rightarrow \Gamma^{\prime}$ one can construct a linear mapping $\widehat{h}: \mathcal{A}(\Gamma) \rightarrow \operatorname{Lin}\left(\mathcal{A}\left(\Gamma^{\prime}\right)\right)$ which behaves nicely with respect to the composition of morphisms and the $*$-operation. This construction depends in a crucial way on Proposition 1.3. Let us briefly describe the construction here. 
Let $a \in E^{\prime},(x, y) \in \Gamma \times_{h} F_{r}(a), \mathfrak{t}_{h}(x, y)=:(x, z)$ and $b:=e_{L}^{\prime}(z)$. Due to Proposition 1.3 we have the following isomorphisms:

$$
\begin{aligned}
& i_{1}: \Omega_{R}^{1 / 2}(x) \otimes \Omega_{R}^{1 / 2}(y) \rightarrow \Omega^{1 / 2} T_{(x, y)}\left(\Gamma \times_{h} F_{r}(a)\right), \\
& \mathfrak{t}_{h}: \Omega^{1 / 2} T_{(x, y)}\left(\Gamma \times_{h} F_{r}(a)\right) \rightarrow \Omega^{1 / 2} T_{(x, z)}\left(\Gamma *_{h} F_{r}(a)\right), \\
& i_{2}: \Omega_{L}^{1 / 2}(x) \otimes \Omega_{R}^{1 / 2}(z) \rightarrow \Omega^{1 / 2} T_{(x, z)}\left(\Gamma *_{h} F_{r}(a)\right) .
\end{aligned}
$$

Thus $\left(i_{2}\right)^{-1} \mathfrak{t}_{h} i_{1}\left(\varrho_{x} \otimes \varrho_{y}\right)=: \lambda_{x} \otimes \varrho_{z}$ for some $\lambda_{x} \otimes \varrho_{z} \in \Omega_{L}^{1 / 2}(x) \otimes \Omega_{R}^{1 / 2}(z)$. Moreover, since the mapping $F_{l}(y) \ni u \mapsto h_{e_{L}^{\prime}(y)}^{R}(x) u \in F_{l}(z)$ is a diffeomorphism, it defines an isomorphism between $\Omega_{L}^{1 / 2}(y)$ and $\Omega_{L}^{1 / 2}(z)$.

Now let $\omega=\lambda \otimes \varrho \in \mathcal{A}(\Gamma), \omega^{\prime}=\lambda^{\prime} \otimes \varrho^{\prime} \in \mathcal{A}\left(\Gamma^{\prime}\right)$. Then $\left(i_{2}\right)^{-1} \mathfrak{t}_{h} i_{1}(\varrho(x) \otimes$ $\left.\varrho^{\prime}(y)\right)=: \widetilde{\lambda}_{x} \otimes \widetilde{\varrho}_{z}$, and $h_{e_{L}^{\prime}(y)}^{R}(x) \lambda^{\prime}(y)=: \widetilde{\lambda}_{z}^{\prime}$, so the expression $\left[\lambda(x) \widetilde{\lambda}_{x}\right] \otimes$ $\widetilde{\lambda}_{z}^{\prime} \otimes \widetilde{\varrho}_{z}$ defines a 1-density on $F_{l}\left(f_{h}(b)\right)$ with values in the one-dimensional vector space $\Omega_{L}^{1 / 2}(z) \otimes \Omega_{R}^{1 / 2}(z)$. In this way we can define the mapping $\widehat{h}$ mentioned above:

$$
\left(\widehat{h}(\omega) \omega^{\prime}\right)(z):=\int_{F_{l}\left(f_{h}(b)\right)}[\lambda \widetilde{\lambda}] \otimes \widetilde{\lambda}_{z}^{\prime} \otimes \widetilde{\varrho}_{z} .
$$

Choose $\omega_{0}=\lambda_{0} \otimes \varrho_{0}$ and $\omega_{0}^{\prime}=\lambda_{0}^{\prime} \otimes \varrho_{0}^{\prime}$. Then $\left(i_{2}\right)^{-1} \mathfrak{t}_{h} i_{1}\left(\varrho_{0}(x) \otimes \varrho_{0}^{\prime}(y)\right)=$ $t_{h}(x, y) \lambda_{0}(x) \otimes \varrho_{0}^{\prime}(z)$ for some smooth, non-vanishing function $t_{h}: \Gamma \times_{h} \Gamma^{\prime}$ $\rightarrow \mathbb{R}$ and $h_{e_{L}^{\prime}(y)}^{R}(x) \lambda_{0}^{\prime}(y)=\lambda_{0}^{\prime}(z)$. For $\omega=f_{1} \omega_{0}$ and $\omega^{\prime}=f_{2} \omega_{0}^{\prime}$ we get the explicit expression

$$
\begin{aligned}
\left(\widehat{h}(\omega) \omega^{\prime}\right)(z) & =\left[\int_{F_{l}\left(f_{h}(b)\right)} \lambda_{0}^{2}(x) f_{1}(x) t_{h}(x, y) f_{2}(y)\right] \omega_{0}^{\prime}(z) \\
& =:\left(f_{1} *_{h} f_{2}\right)(z) \omega_{0}^{\prime}(z),
\end{aligned}
$$

where $y$ is defined by $\mathfrak{t}_{h}(x, y)=(x, z)$, i.e. $y=s^{\prime}\left(h_{b}^{L}(x)\right) z$.

Now taking $h=$ id $: \Gamma \rightarrow \Gamma$ we get an algebra structure on $\mathcal{A}(\Gamma)$ : the formula for the product is $\omega_{1} \omega_{2}=:\left(f_{1} * f_{2}\right) \omega_{0}$ where

$$
\left(f_{1} * f_{2}\right)(x):=\int_{F_{l}(x)} \lambda_{0}^{2}(y) f_{1}(y) f_{2}(s(y) x)=\int_{F_{r}(x)} \varrho_{0}^{2}(y) f_{1}(x s(y)) f_{2}(y) .
$$

It seems that there is no natural, geometric, norm on $\mathcal{A}(\Gamma)$, but one can introduce a family of useful norms "indexed" by $\omega_{0}$ 's $[11,13]$. A chosen $\lambda_{0}$ defines $\omega_{0}$ and any bidensity can be written as $\omega=f \omega_{0}$. Define

$$
\begin{aligned}
\|\omega\|_{l} & :=\sup _{a \in \Gamma^{0}} \int_{F_{l}(a)} \lambda_{0}^{2}|f|, \quad\|\omega\|_{r}:=\sup _{a \in \Gamma^{0}} \int_{F_{r}(a)} \varrho_{0}^{2}|f|, \\
\|\omega\|_{0} & :=\max \left\{\|\omega\|_{l},\|\omega\|_{r}\right\} .
\end{aligned}
$$


It can be shown that $\|\omega\|_{l},\|\omega\|_{r},\|\omega\|_{0}$ are norms, and $\left(\mathcal{A}(\Gamma), *,\|\cdot\|_{0}\right)$ is a normed $*$-algebra.

Representation of the algebra of a groupoid associated with a morphism. Let $\Psi$ be a smooth half-density on $\Gamma^{\prime}$ with compact support and $\omega \in \mathcal{A}(\Gamma)$, $\omega=\lambda \otimes \varrho$. Let $(x, y) \in \Gamma \times_{h} \Gamma^{\prime}$ and $\mathfrak{t}_{h}(x, y)=:(x, z)$. As in the definition of $\widehat{h}, \varrho(x) \otimes \Psi(y)$ can be viewed as a half-density on $T_{(x, y)}\left(\Gamma \times_{h} \Gamma^{\prime}\right)$ and $\mathfrak{t}_{h}(\varrho(x) \otimes \Psi(y))$ is a half-density on $T_{(x, z)}\left(\Gamma *_{h} \Gamma^{\prime}\right)$. Since $\Omega_{L}^{1 / 2}(x) \otimes \Omega^{1 / 2} T_{z} \Gamma^{\prime} \simeq$ $\Omega^{1 / 2} T_{(x, z)}\left(\Gamma *_{h} \Gamma^{\prime}\right)$ this half-density can be written as $\widetilde{\lambda}_{x} \otimes \Psi_{x}(z)$, where $\widetilde{\lambda}_{x}$ is a half-density on $T_{x}^{l}(\Gamma)$ and $\Psi_{x}(z)$ a half-density on $T_{z}\left(\Gamma^{\prime}\right)$. Then $\lambda(x) \widetilde{\lambda}_{x} \otimes \Psi_{x}(z)$ is a 1 -density on $T_{x}^{l} \Gamma$ with values in half-densities on $T_{z}\left(\Gamma^{\prime}\right)$. Integrating $\lambda(x) \widetilde{\lambda}_{x} \otimes \Psi_{x}(z)$ we get a half-density on $T_{z}\left(\Gamma^{\prime}\right)$. In this way we get an operator $\pi_{h}(\omega)$ for $\omega \in \mathcal{A}(\Gamma)$ defined on a dense linear subspace of $L^{2}\left(\Gamma^{\prime}\right)$,

$$
\left(\pi_{h}(\omega) \Psi\right)(z):=\int_{F_{l}\left(f_{h}(b)\right)}[\lambda(x) \tilde{\lambda}(x)] \otimes \Psi_{x}(z) .
$$

Choose $\omega_{0}$ and write $\omega=f \omega_{0}$. Since $e_{R}^{\prime}$ is a surjective submersion, there is a natural isomorphism $\Omega^{1 / 2} T_{w} \Gamma^{\prime} \simeq \Omega_{R}^{1 / 2}(w) \otimes \Omega^{1 / 2} T_{e_{R}^{\prime}(w)} E^{\prime}$ for any $w \in \Gamma^{\prime}$. Therefore, if we choose $\varrho_{0}^{\prime}$ and a non-vanishing, real half-density $\nu_{0}$ on $E^{\prime}$, then $\varrho_{0}^{\prime} \otimes \nu_{0}$ defines a non-vanishing, real half-density on $\Gamma^{\prime}$. So any other smooth half-density $\Psi$ with compact support can be written as $\Psi=\psi \varrho_{0}^{\prime} \otimes \nu_{0}=: \psi \Psi_{0}$ for some smooth, complex-valued function $\psi$ with compact support. It is easy to see that $\mathfrak{t}_{h}\left(\varrho_{0}(x) \otimes \varrho_{0}^{\prime}(y) \otimes \nu_{0}(a)\right)=t_{h}(x, y)\left(\lambda_{0}(x) \otimes\right.$ $\left.\varrho_{0}^{\prime}(z) \otimes \nu_{0}(a)\right)$, where $t_{h}$ is as in the definition of $\widehat{h}$. So the explicit formula is

$$
\left(\pi_{h}(\omega) \Psi\right)(z)=\left[\int_{F_{l}\left(f_{h}(b)\right)} \lambda_{0}^{2}(x) f(x) t_{h}(x, y) \psi(y)\right] \Psi_{0}(z),
$$

where $b:=e_{L}^{\prime}(z)$ and $\mathfrak{t}_{h}(x, y)=(x, z)$. In the next proposition we collect the essential properties of the mappings $\widehat{h}$ and $\pi_{h}$.

Proposition 1.5 ([13]). Let $\Gamma, \Gamma^{\prime}, \Gamma^{\prime \prime}$ be differential groupoids, and let $h: \Gamma \rightarrow \Gamma^{\prime}$ and $k: \Gamma^{\prime} \rightarrow \Gamma^{\prime \prime}$ be differential groupoid morphisms. For any $\omega \in \mathcal{A}(\Gamma), \omega^{\prime}, \omega_{1}^{\prime} \in \mathcal{A}\left(\Gamma^{\prime}\right)$ and $\omega^{\prime \prime} \in \mathcal{A}\left(\Gamma^{\prime \prime}\right)$ :

(a) $\widehat{k}\left(\left(\widehat{h}(\omega) \omega^{\prime}\right)\right) \omega^{\prime \prime}=\widehat{k h}(\omega)\left(\widehat{k}\left(\omega^{\prime}\right) \omega^{\prime \prime}\right)$.

(b) $\pi_{k}\left(\widehat{h}(\omega) \omega^{\prime}\right)=\pi_{k h}(\omega) \pi_{k}\left(\omega^{\prime}\right)$.

(c) $\left(\omega^{\prime}\right)^{*}\left(\widehat{h}(\omega) \omega_{1}^{\prime}\right)=\left(\widehat{h}\left(\omega^{*}\right) \omega^{\prime}\right)^{*} \omega_{1}^{\prime}$.

(d) $\pi_{h}$ is a *-representation of $\mathcal{A}(\Gamma)$ by bounded operators on $L^{2}\left(\Gamma^{\prime}\right)$, and for any $\omega_{0}$ we have the estimate $\left\|\pi_{h}(\omega)\right\| \leq\|\omega\|_{0}$ (the norm on the right hand side is defined in (3)). 
(e) Choose some $*$-invariant $\omega_{0}^{\prime}$ and let $\|\cdot\|_{0}^{\prime}$ denote the associated norm. For any $\omega^{\prime} \in \mathcal{A}\left(\Gamma^{\prime}\right)$ there exists a sequence $\omega_{n} \in \mathcal{A}(\Gamma)$ such that $\lim _{n \rightarrow \infty}\left\|\widehat{h}\left(\omega_{n}\right) \omega^{\prime}-\omega^{\prime}\right\|_{0}^{\prime}=0$.

Due to these properties one can define a $C^{*}$-norm on $\mathcal{A}(\Gamma)$, then complete $\mathcal{A}(\Gamma)$ in this norm and get a kind of universal $C^{*}$-algebra of the differential groupoid. For any morphism $h$, the mapping $\widehat{h}$ can be uniquely extended to a morphism between the corresponding $C^{*}$-algebras, and $\pi_{h}$ to a nondegenerate representation [13].

It is also easy to see that the representation $\pi_{\text {id }}$ is faithful on $\mathcal{A}(\Gamma)$, so the function $\omega \mapsto\left\|\pi_{\text {id }}(\omega)\right\|$ defines a $C^{*}$-norm on $\mathcal{A}(\Gamma)$. The completion of $\mathcal{A}(\Gamma)$ in this norm is called the reduced $C^{*}$-algebra of $\Gamma$ and will be denoted by $C_{r}^{*}(\Gamma)$.

Bisections and their action on groupoid algebras. Recall that a submanifold $B \subset \Gamma$ is a bisection iff $\left.e_{L}\right|_{B}$ and $\left.e_{R}\right|_{B}$ are diffeomorphisms. The set of bisections of a groupoid $\Gamma$ is a group under the natural multiplication of subsets of $\Gamma$ and inverse given by $B^{-1}:=s(B)$. Bisections act (from the left) on $\Gamma$ by diffeomorphisms: for $g \in \Gamma, B g=g^{\prime} g$, where $g^{\prime}$ is the unique point in $B$ composable with $g$. One can immediately verify that $B F_{r}(g)=F_{r}(g)$ and $B F_{l}(g)=F_{l}(B g)$. These facts enable us to define an action of bisections on $\mathcal{A}(\Gamma)$ according to the formula

$(B \omega)(B g)(B v \otimes B w):=\omega(g)(v \otimes w)$, where $v \in \Lambda^{\max } T_{g}^{l} \Gamma, w \in \Lambda^{\max } T_{g}^{r} \Gamma$. It is easy to see that for $\omega_{0}=\lambda_{0} \otimes \varrho_{0}$ and $B \omega_{0}=: f \omega_{0}$, the function $f$ is given by

$$
f(B g) \varrho_{0}(B g)(B w)=\varrho_{0}(g)(w), \quad w \in \Lambda^{\max } T_{g}^{r} \Gamma .
$$

It turns out that if $B$ is a bisection of $\Gamma$ and $h: \Gamma \rightarrow \Gamma^{\prime}$ is a morphism, then the set $h(B)$ is a bisection of $\Gamma^{\prime}$. In the next lemma we collect the basic properties of the action of morphisms on bisections which allow us to interpret bisections as multipliers on $C^{*}(\Gamma)$ and $C_{r}^{*}(\Gamma)$.

Proposition 1.6 ([13]). Let $\Gamma, \Gamma^{\prime}$ be differential groupoids, $B$ a bisection of $\Gamma$ and $h: \Gamma \rightarrow \Gamma^{\prime}$ a morphism. For any $\omega, \omega_{1} \in \mathcal{A}(\Gamma)$ and $\omega^{\prime} \in$ $\mathcal{A}\left(\Gamma^{\prime}\right)$ :

1. $\omega^{*}\left(B \omega_{1}\right)=(s(B) \omega)^{*} \omega_{1}$.

2. $(\widehat{h}(B \omega)) \omega^{\prime}=h(B)\left(\widehat{h}(\omega) \omega^{\prime}\right)$.

3. $\pi_{h}(B \omega)=h(B) \pi_{h}(\omega)$.

\section{2. $C^{*}$-algebra of the Cartesian product of differential groupoids.}

In this section we collect some technical results about the $C^{*}$-algebra of the Cartesian product of differential groupoids. We are not able to prove that $C^{*}\left(\Gamma_{1} \times \Gamma_{2}\right)$ is equal to (some kind of) $C^{*}\left(\Gamma_{1}\right) \otimes C^{*}\left(\Gamma_{2}\right)$. However, we have the following (expected) result. 
Proposition 2.1. Let $\Gamma_{1}, \Gamma_{2}$ be differential groupoids. Then $C_{r}^{*}\left(\Gamma_{1} \times\right.$ $\left.\Gamma_{2}\right)=C_{r}^{*}\left(\Gamma_{1}\right) \otimes_{\sigma} C_{r}^{*}\left(\Gamma_{2}\right)$, where $\otimes_{\sigma}$ denotes the minimal tensor product.

Proof. We adopt the following notation: $\mathcal{A}_{i}:=\mathcal{A}\left(\Gamma_{i}\right), H_{i}:=L^{2}\left(\Gamma_{i}\right)$, $\pi_{i}: \mathcal{A}_{i} \rightarrow B\left(H_{i}\right)$ the identity representation of $\mathcal{A}_{i},\|\cdot\|_{i}$ the operator norm on $B\left(H_{i}\right), A_{i}:=C_{r}^{*}\left(\Gamma_{i}\right)=\overline{\pi_{i}\left(\mathcal{A}_{i}\right)} \|^{\|}, i=1,2$, and the corresponding objects for $\Gamma_{1} \times \Gamma_{2}: \mathcal{A}:=\mathcal{A}\left(\Gamma_{1} \times \Gamma_{2}\right), H:=L^{2}\left(\Gamma_{1} \times \Gamma_{2}\right)=H_{1} \otimes H_{2}, \pi: \mathcal{A} \rightarrow B(H)$, $\|\cdot\|$ the operator norm on $B(H)$ and $A:=C_{r}^{*}\left(\Gamma_{1} \times \Gamma_{2}\right)=\overline{\pi(\mathcal{A})}\|\cdot\|$. It is clear that $\mathcal{A}_{1} \otimes \mathcal{A}_{2} \subset \mathcal{A}$ and $\left.\pi\right|_{\mathcal{A}_{1} \otimes \mathcal{A}_{2}}=\pi_{1} \otimes \pi_{2}$.

Choose non-vanishing, real, left-invariant half-densities $\lambda_{0}$ and $\widetilde{\lambda}_{0}$ along left fibers on $\Gamma_{1}$ and $\Gamma_{2}$ respectively. Then $\lambda_{0} \otimes \widetilde{\lambda}_{0}$ is a non-vanishing, real, left-invariant half-density along left fibers on $\Gamma_{1} \times \Gamma_{2}$, and we have the corresponding right-invariant half-densities and $*$-invariant bidensities: $\omega_{0}^{1}=\lambda_{0} \otimes \varrho_{0}, \omega_{0}^{2}=\widetilde{\lambda}_{0} \otimes \widetilde{\varrho}_{0}$ and $\omega_{0}=\omega_{0}^{1} \otimes \omega_{0}^{2}$. Let $\|\cdot\|_{0}$ be the corresponding norm on $\mathcal{A}$. We begin with the following

Lemma 2.2. $\mathcal{A}_{1} \otimes \mathcal{A}_{2}$ is dense in $\mathcal{A}$ in the topology defined by $\|\cdot\|_{0}$.

Proof. Let $\mathcal{D}(\cdot)$ denote the compactly supported, smooth functions. The following result is standard: for any $f \in \mathcal{D}\left(\Gamma_{1} \times \Gamma_{2}\right)$ there exist compact sets $K_{1} \subset \Gamma_{1}$ and $K_{2} \subset \Gamma_{2}$ such that

$\forall \varepsilon>0 \exists g \in \mathcal{D}\left(\Gamma_{1}\right) \otimes \mathcal{D}\left(\Gamma_{2}\right): \operatorname{supp}(g) \subset K_{1} \times K_{2}$ and $\sup |f-g|<\varepsilon$.

Let $\omega \in \mathcal{A}\left(\Gamma_{1} \times \Gamma_{2}\right)$. Then $\omega=f \omega_{0}$ for some $f \in \mathcal{D}\left(\Gamma_{1} \times \Gamma_{2}\right)$. Let $K_{1}, K_{2}$ be subsets as above, choose some $h \in \mathcal{D}\left(\Gamma_{1} \times \Gamma_{2}\right)$ such that $h(x) \geq 0, h=1$ on $K_{1} \times K_{2}$, and let $M:=\left\|h \omega_{0}\right\|_{0}$. Take $\varepsilon>0$ and let $g=\sum f_{i} \otimes k_{i} \in$ $\mathcal{D}\left(\Gamma_{1}\right) \otimes \mathcal{D}\left(\Gamma_{2}\right)$ be such that $\sup |f-g|<\varepsilon / M$. Define $\omega_{i}^{1}:=f_{i} \omega_{0}^{1}$ and $\omega_{i}^{2}:=k_{i} \omega_{0}^{2}$. Then simple computations show that $\left\|\omega-\sum \omega_{i}^{1} \otimes \omega_{i}^{2}\right\|_{0} \leq \varepsilon$.

Since $\|\omega\| \leq\|\omega\|_{0}$, for $\omega \in \mathcal{A}$ the closure of $\pi\left(\mathcal{A}_{1} \otimes \mathcal{A}_{2}\right)$ contains the closure of $\pi(\mathcal{A})$, i.e.

$$
A \subset \overline{\pi\left(\mathcal{A}_{1} \otimes \mathcal{A}_{2}\right)}\|\cdot\| \overline{\pi_{1}\left(\mathcal{A}_{1}\right) \otimes \pi_{2}\left(\mathcal{A}_{2}\right)}\|\cdot\|
$$

This means that $A \subset A_{1} \otimes_{\sigma} A_{2}$. On the other hand, for $A_{1} \ni a_{1}=\lim \omega_{n}$ with $\omega_{n} \in \mathcal{A}_{1}$ and $A_{2} \ni a_{2}=\lim \omega_{n}^{\prime}$ with $\omega_{n}^{\prime} \in \mathcal{A}_{2}$ we immediately verify that $\pi_{1}\left(a_{1}\right) \otimes \pi_{2}\left(a_{2}\right)=\lim \pi_{1}\left(a_{1}\right) \otimes \pi_{2}\left(\omega_{n}^{\prime}\right)=\lim \pi\left(\omega_{n} \otimes \omega_{n}^{\prime}\right)$. In this way $\pi_{1}\left(A_{1}\right) \otimes \pi_{2}\left(A_{2}\right) \subset \overline{\pi(\mathcal{A})}=C_{r}^{*}\left(\Gamma_{1} \times \Gamma_{2}\right)$ and

$$
A_{1} \otimes_{\sigma} A_{2}=\overline{\pi_{1}\left(A_{1}\right) \otimes \pi_{2}\left(A_{2}\right)} \subset C_{r}^{*}\left(\Gamma_{1} \times \Gamma_{2}\right) .
$$

From now on, if $A$ and $B$ are $C^{*}$-algebras, a tensor product $A \otimes B$ means the minimal tensor product.

In the next lemma $\omega\left(\omega_{1} \otimes I\right)$ and $\omega\left(I \otimes \omega_{1}\right)$ mean products in $M\left(C_{r}^{*}(\Gamma \times \Gamma)\right)$ (i.e. the multiplier algebra). 
Lemma 2.3. Let $\omega=F\left(\omega_{0} \otimes \omega_{0}\right) \in \mathcal{A}(\Gamma \times \Gamma)$ and $\omega_{1}=f \omega_{0} \in \mathcal{A}(\Gamma)$. Then $\omega\left(\omega_{1} \otimes I\right)$ and $\omega\left(I \otimes \omega_{1}\right)$ are elements of $\mathcal{A}(\Gamma \times \Gamma)$ given by $\omega\left(\omega_{1} \otimes I\right)=:(F *(f \otimes I))\left(\omega_{0} \otimes \omega_{0}\right), \quad \omega\left(I \otimes \omega_{1}\right)=:(F *(I \otimes f))\left(\omega_{0} \otimes \omega_{0}\right)$, where

$$
\begin{aligned}
& (F *(f \otimes I))\left(g_{1}, g_{2}\right):=\int_{F_{l}\left(g_{1}\right)} \lambda_{0}^{2}(g) F\left(g, g_{2}\right) f\left(s(g) g_{1}\right), \\
& (F *(I \otimes f))\left(g_{1}, g_{2}\right):=\int_{F_{l}\left(g_{2}\right)} \lambda_{0}^{2}(g) F\left(g_{1}, g\right) f\left(s(g) g_{2}\right) .
\end{aligned}
$$

Proof. It is enough to show that

$$
\begin{aligned}
& (F *(f \otimes I)) *\left(f_{1} \otimes f_{2}\right)=F *\left(f * f_{1} \otimes f_{2}\right), \\
& (F *(I \otimes f)) *\left(f_{1} \otimes f_{2}\right)=F *\left(f_{1} \otimes f * f_{2}\right) .
\end{aligned}
$$

Let us compute the LHS of the first equality:

$$
\begin{aligned}
& (F *(f \otimes I)) *\left(f_{1} \otimes f_{2}\right)\left(g_{1}, g_{2}\right) \\
& =\int_{F_{l}\left(g_{1}\right) \times F_{l}\left(g_{2}\right)}\left(\lambda_{0}^{2}(g) \otimes \lambda_{0}^{2}(h)\right)(F *(f \otimes I))(g, h) f_{1}\left(s(g) g_{1}\right) f_{2}\left(s(h) g_{2}\right) \\
& =\int_{F_{l}\left(g_{1}\right) \times F_{l}\left(g_{2}\right)}\left(\lambda_{0}^{2}(g) \otimes \lambda_{0}^{2}(h)\right) f_{1}\left(s(g) g_{1}\right) f_{2}\left(s(h) g_{2}\right) \int_{F_{l}(g)} \lambda_{0}^{2}(k) F(k, h) f(s(k) g) .
\end{aligned}
$$

Since $F_{l}(g)=F_{l}\left(g_{1}\right)$ the above integral is equal to

$$
\int_{F_{l}\left(g_{1}\right) \times F_{l}\left(g_{1}\right) \times F_{l}\left(g_{2}\right)}\left(\lambda_{0}^{2}(g) \otimes \lambda_{0}^{2}(k) \otimes \lambda_{0}^{2}(h)\right) f_{1}\left(s(g) g_{1}\right) f_{2}\left(s(h) g_{2}\right) F(k, h) f(s(k) g) .
$$

And the RHS:

$$
\begin{array}{rl}
F & *\left(f * f_{1} \otimes f_{2}\right)\left(g_{1}, g_{2}\right) \\
= & \int_{F_{l}\left(g_{1}\right) \times F_{l}\left(g_{2}\right)}\left(\lambda_{0}^{2}(g) \otimes \lambda_{0}^{2}(h)\right) F(g, h)\left(f * f_{1}\right)\left(s(g) g_{1}\right) f_{2}\left(s(h) g_{2}\right) \\
= & \int_{F_{l}\left(g_{1}\right) \times F_{l}\left(g_{2}\right)}\left(\lambda_{0}^{2}(g) \otimes \lambda_{0}^{2}(h)\right) F(g, h) f_{2}\left(s(h) g_{2}\right) \int_{F_{r}\left(g_{1}\right)} \varrho_{0}^{2}(k) f\left(s(g) g_{1} s(k)\right) f_{1}(k) \\
= & \int_{F_{l}\left(g_{1}\right) \times F_{l}\left(g_{2}\right)}\left(\lambda_{0}^{2}(g) \otimes \lambda_{0}^{2}(h)\right) F(g, h) f_{2}\left(s(h) g_{2}\right) \\
& \times \int_{F_{l}\left(e_{R}\left(g_{1}\right)\right)} \lambda_{0}^{2}(k) f\left(s(g) g_{1} k\right) f_{1}(s(k))
\end{array}
$$




$$
\begin{aligned}
& \left.=\int_{F_{l}\left(g_{1}\right) \times F_{l}\left(g_{2}\right)}\left(\lambda_{0}^{2}(g) \otimes \lambda_{0}^{2}(h)\right) F(g, h) f_{2}\left(s(h) g_{2}\right) \int_{F_{l}\left(g_{1}\right)} \lambda_{0}^{2}(k) f(s(g) k)\right) f_{1}\left(s(k) g_{1}\right) \\
& =\int_{F_{l}\left(g_{1}\right) \times F_{l}\left(g_{1}\right) \times F_{l}\left(g_{2}\right)}\left(\lambda_{0}^{2}(k) \otimes \lambda_{0}^{2}(g) \otimes \lambda_{0}^{2}(h)\right) f_{1}\left(s(k) g_{1}\right) f_{2}\left(s(h) g_{2}\right) F(g, h) f(s(g) k) ;
\end{aligned}
$$

the passage from the second to the third line follows from the fact that $s$ restricted to $F_{r}\left(g_{1}\right)$ is a diffeomorphism onto $F_{l}\left(e_{R}\left(g_{1}\right)\right)$, and $s\left(\varrho_{0}\right)=\lambda_{0}$; the equality of the third and fourth lines is a consequence of the diffeomorphism $F_{l}\left(e_{R}\left(g_{1}\right)\right) \ni k \mapsto g_{1} k \in F_{l}\left(g_{1}\right)$.

The equality $(F *(I \otimes f)) *\left(f_{1} \otimes f_{2}\right)=F *\left(f_{1} \otimes f * f_{2}\right)$ can be proved in the same way.

Later on we will need the following technical result:

Lemma 2.4. Let $\Phi: \Gamma \times \Gamma \rightarrow \Gamma \times \Gamma$ be a diffeomorphism, and $\psi: \Gamma \times \Gamma$ $\rightarrow \mathbb{R}$ be a smooth, non-vanishing function. Let $\lambda_{0}$ be a left-invariant, real, non-vanishing half-density and $\omega_{0}$ the corresponding $*$-invariant bidensity. Then $\lambda_{0} \otimes \lambda_{0}$ is a left-invariant, real, non-vanishing half-density on $\Gamma \times \Gamma$ and $\omega_{0} \otimes \omega_{0}$ is the corresponding $*$-invariant bidensity. Let $\|\cdot\|_{l},\|\cdot\|_{r},\|\cdot\|_{0}$ be the norms on $\mathcal{A}(\Gamma \times \Gamma)$ associated with $\lambda_{0} \otimes \lambda_{0}$ as defined in (3). Define $S: \mathcal{A}(\Gamma) \otimes \mathcal{A}(\Gamma) \rightarrow \mathcal{A}(\Gamma \times \Gamma)$ by

$$
S\left(\sum \omega_{i} \otimes \omega_{i}^{\prime}\right):=\psi \Phi\left(\sum f_{i} \otimes f_{i}^{\prime}\right)\left(\omega_{0} \otimes \omega_{0}\right),
$$

where $\omega_{i}=: f_{i} \omega_{0}, \omega_{i}^{\prime}=: f_{i}^{\prime} \omega_{0}$ and $(\Phi g)(x):=g\left(\Phi^{-1}(x)\right)$ is the push-forward of the function $g$. Then $S(\mathcal{A}(\Gamma) \otimes \mathcal{A}(\Gamma))$ is dense in $\mathcal{A}(\Gamma \times \Gamma)$ in the topology defined by $\|\cdot\|_{0}$.

Proof. Let $\omega=: F\left(\omega_{0} \otimes \omega_{0}\right) \in \mathcal{A}(\Gamma \times \Gamma)$ and $K:=\operatorname{supp} F$. Define $\widetilde{F}:=F / \psi$ and $F_{1}:=\Phi^{-1} \widetilde{F}$. Then $\operatorname{supp} F_{1}=\Phi^{-1}(K)$ is a compact set. There exist compact sets $K_{1}, K_{2}$ such that $\Phi^{-1}(K) \subset K_{1} \times K_{2}$ and

$$
\forall \varepsilon \exists f_{i}, g_{i}: \operatorname{supp} f_{i} \subset K_{1}, \operatorname{supp} g_{i} \subset K_{2}, \sup \left|F_{1}-\sum f_{i} \otimes g_{i}\right| \leq \varepsilon .
$$

Then $\operatorname{supp} \Phi\left(\sum f_{i} \otimes g_{i}\right) \subset \Phi\left(K_{1} \times K_{2}\right)$. Choose a smooth function $h \in$ $\mathcal{D}(\Gamma \times \Gamma)$ such that $0 \leq h \leq 1$ and $h=1$ on $K \cup \Phi\left(K_{1} \times K_{2}\right)$. Let $M:=\|h\|_{0}$ and $N:=\sup \left\{\left|\psi\left(g_{1}, g_{2}\right)\right|:\left(g_{1}, g_{2}\right) \in K \cup \Phi\left(K_{1} \times K_{2}\right)\right\}$. Let $\varepsilon>0$ be given and let $f_{i}, g_{i}$ be as above, and $\sup \left|F_{1}-\sum f_{i} \otimes g_{i}\right| \leq \varepsilon / M N$. Now for $\widetilde{\omega}:=\sum f_{i} \omega_{0} \otimes g_{i} \omega_{0}$ we have

$$
\begin{aligned}
& \|\omega-S(\widetilde{\omega})\|_{l} \\
& =\sup _{\left(e_{1}, e_{2}\right) \in E \times E} \int_{F_{l}\left(e_{1}, e_{2}\right)} \lambda_{0}^{2}\left(g_{1}\right) \otimes \lambda_{0}^{2}\left(g_{2}\right)\left|F\left(g_{1}, g_{2}\right)-\psi\left(g_{1}, g_{2}\right) \Phi\left(\sum f_{i} \otimes g_{i}\right)\left(g_{1}, g_{2}\right)\right| .
\end{aligned}
$$


We can estimate the integral as follows:

$$
\begin{aligned}
\int_{F_{l}\left(e_{1}, e_{2}\right)} \lambda_{0}^{2}\left(g_{1}\right) \otimes & \lambda_{0}^{2}\left(g_{2}\right)\left|F\left(g_{1}, g_{2}\right)-\psi\left(g_{1}, g_{2}\right) \Phi\left(\sum f_{i} \otimes g_{i}\right)\left(g_{1}, g_{2}\right)\right| \\
= & \int_{F_{l}\left(e_{1}, e_{2}\right)} \lambda_{0}^{2}\left(g_{1}\right) \otimes \lambda_{0}^{2}\left(g_{2}\right)\left|h\left(g_{1}, g_{2}\right)\right| \\
& \times\left|F\left(g_{1}, g_{2}\right)-\psi\left(g_{1}, g_{2}\right) \Phi\left(\sum f_{i} \otimes g_{i}\right)\left(g_{1}, g_{2}\right)\right| \\
= & \int_{F_{l}\left(e_{1}, e_{2}\right)} \lambda_{0}^{2}\left(g_{1}\right) \otimes \lambda_{0}^{2}\left(g_{2}\right)\left|h\left(g_{1}, g_{2}\right)\right| \\
& \times\left|\psi\left(g_{1}, g_{2}\right)\right|\left|\widetilde{F}\left(g_{1}, g_{2}\right)-\Phi\left(\sum f_{i} \otimes g_{i}\right)\left(g_{1}, g_{2}\right)\right| \\
\leq & N \sup \left|\widetilde{F}-\Phi\left(\sum f_{i} \otimes g_{i}\right)\right| \int_{F_{l}\left(e_{1}, e_{2}\right)} \lambda_{0}^{2}\left(g_{1}\right) \otimes \lambda_{0}^{2}\left(g_{2}\right)\left|h\left(g_{1}, g_{2}\right)\right| .
\end{aligned}
$$

Since

$$
\begin{aligned}
\sup \left|\widetilde{F}-\Phi\left(\sum f_{i} \otimes g_{i}\right)\right| & =\sup \left|\left(\Phi F_{1}\right)-\Phi\left(\sum f_{i} \otimes g_{i}\right)\right| \\
& =\sup \left|F_{1}-\sum f_{i} \otimes g_{i}\right| \leq \varepsilon / M N .
\end{aligned}
$$

we get $\|\omega-S(\widetilde{\omega})\|_{l} \leq \varepsilon$. In the same way we can estimate $\|\omega-S(\widetilde{\omega})\|_{r}$.

3. Double Lie groups and related objects. In this section we introduce basic objects of our investigations: groupoids related to a double Lie group (DLG). We begin by recalling the definition of a DLG (also known as a matched pair of Lie groups or a bicrossproduct Lie group).

Definition $3.1([6])$. A double Lie group is a triple $(G ; A, B)$ of Lie groups such that $A, B$ are closed subgroups of $G, A \cap B=\{e\}$ and $G=A B$.

The structure of a DLG defines four projections:

$$
a_{L}, a_{R}: G \rightarrow A, \quad b_{L}, b_{R}: G \rightarrow B \quad \text { by } g=a_{L}(g) b_{R}(g)=b_{L}(g) a_{R}(g) .
$$

We also define a relation $m_{A}: G \times G \rightarrow G$ by

$$
\operatorname{Gr}\left(m_{A}\right):=\left\{\left(b_{1} a b_{2} ; b_{1} a, a b_{2}\right): a \in A, b_{1}, b_{2} \in B\right\},
$$

and a diffeomorphism

$$
s_{A}: G \ni g \mapsto a_{R}(g) b_{R}(g)^{-1}=b_{L}(g)^{-1} a_{L}(g) \in G .
$$

It turns out [17] that $G_{A}:=\left(G, m_{A}, A, s_{A}\right)$ is a differential groupoid. The same is true for $G_{B}:=\left(G, m_{B}, B, s_{B}\right)$, where $m_{B}, s_{B}$ are defined similarly to $m_{A}, s_{A}$. Moreover $\delta:=m_{B}^{T}: G_{A} \rightarrow G_{A} \times G_{A}$ and $s_{B}: G_{A} \rightarrow G_{A}$ are morphisms of differential groupoids. In the "extreme case" $A=\{e\}, B=G$ we have $G_{A}=G$ (the groupoid $G_{A}$ is a group) and $G_{B}=\left(G, \operatorname{diag}^{T}, G\right.$,id), 
where diag : $G \ni g \mapsto(g, g) \in G \times G$ is the diagonal mapping. The basic example of a DLG the reader may think of is an Iwasawa decomposition of a semisimple Lie group $G=K(A N)$.

In the next lemma we explicitly describe the mappings and sets related to the morphism $\delta$. We use the same notation as in Proposition 1.3. The proof is straightforward.

Lemma 3.2. Let $(G ; A, B)$ be a double Lie group and let $\delta:=m_{B}^{T}$.

1. $\operatorname{Gr}(\delta)=\left\{\left(a_{2} b_{2}, b_{2} a_{3} ; a_{2} b_{2} a_{3}\right): a_{2}, a_{3} \in A, b_{2} \in B\right\}$.

2. $f_{\delta}: A \times A \ni\left(a_{1}, a_{2}\right) \mapsto a_{1} a_{2} \in A$.

3. $G_{A} \times_{\delta}\left(G_{A} \times G_{A}\right)=\left\{\left(\widetilde{b}_{1} a_{2} a_{3} ; a_{2} b_{2}, a_{3} b_{3}\right): \widetilde{b}_{1}, b_{2}, b_{3} \in B, a_{2}, a_{3} \in A\right\}$.

4. $G_{A} \times_{\delta} F_{r}\left(\widetilde{a}_{2}, \widetilde{a}_{3}\right)=\left\{\left(\widetilde{b}_{1} a_{L}\left(\widetilde{b}_{2} \widetilde{a}_{2}\right) a_{L}\left(\widetilde{b}_{3} \widetilde{a}_{3}\right) ; \widetilde{b}_{2} \widetilde{a}_{2}, \widetilde{b}_{2} \widetilde{a}_{2}\right): \widetilde{b}_{1}, \widetilde{b}_{2}, \widetilde{b}_{3} \in B\right\}$.

5. $G_{A} *_{\delta}\left(G_{A} \times G_{A}\right)=\left\{\left(a_{2} a_{3} b_{1} ; a_{2} b_{2}, a_{3} b_{3}\right): b_{1}, b_{2}, b_{3} \in B, a_{2}, a_{3} \in A\right\}$;

6. $G_{A} *_{\delta} F_{r}\left(\widetilde{a}_{2}, \widetilde{a}_{3}\right)=\left\{\left(a_{L}\left(\widetilde{b}_{2} \widetilde{a}_{2}\right) a_{L}\left(\widetilde{b}_{3} \widetilde{a}_{3}\right) b_{1} ; \widetilde{b}_{2} \widetilde{a}_{2}, \widetilde{b}_{2} \widetilde{a}_{2}\right): b_{1}, \widetilde{b}_{2}, \widetilde{b}_{3} \in B\right\}$.

7. $\delta^{L}\left(g_{1} ; a_{2}, a_{3}\right)=\left(a_{2} b_{L}\left(a_{3} b_{R}\left(g_{1}\right)\right), a_{3} b_{R}\left(g_{1}\right)\right), a_{L}\left(g_{1}\right)=a_{2} a_{3}$.

8. $\delta^{R}\left(g_{1} ; a_{2}, a_{3}\right)=\left(b_{L}\left(g_{1}\right) a_{2}, b_{R}\left(b_{L}\left(g_{1}\right) a_{2}\right) a_{3}\right), a_{R}\left(g_{1}\right)=a_{2} a_{3}$.

9. $m_{\delta}\left(g_{1} ; g_{2}, g_{3}\right)=\left(b_{L}\left(g_{1}\right) g_{2}, b_{R}\left(b_{L}\left(g_{1}\right) a_{L}\left(g_{2}\right)\right) g_{3}\right)$.

10. $\mathfrak{t}_{\delta}\left(g_{1} ; g_{2}, g_{3}\right)=\left(g_{1} ; b_{L}\left(g_{1}\right) g_{2}, b_{R}\left(b_{L}\left(g_{1}\right) a_{L}\left(g_{2}\right)\right) g_{3}\right)$.

For any DLG one can define the pentagonal diffeomorphism

$$
W: G \times G \ni(s, t) \mapsto W(s, t):=\left(s a_{L}(t)^{-1}, b_{R}\left(s a_{L}(t)^{-1}\right) t\right) \in G \times G .
$$

Then $W^{-1}(s, t)=\left(s a_{L}\left(b_{R}(s)^{-1} t\right), b_{R}(s)^{-1} t\right)$. By push-forward of halfdensities, $W$ defines a multiplicative unitary operator on $L^{2}(G) \otimes L^{2}(G)$, which will also be denoted by $W$. Then $W^{*}$ is the push-forward by $W^{-1}$.

Now we give an interpretation of $W$ in terms of the groupoids $G_{A}$ and $G_{B}$. We need this to prove easily that $W \in M\left(C B\left(L^{2}(G)\right) \otimes C_{r}^{*}\left(G_{A}\right)\right)$ $(C B(H)$ stands for compact operators). However, this interpretation can also be used to show that $W$ is a unitary bicharacter on quantum groups with some universal properties and to construct a quantum double. For a groupoid $\Gamma=(\Gamma, m, E, s)$ we denote by $\Gamma^{\mathrm{op}}$ the groupoid with the reverse multiplication, i.e. $\left(\Gamma, m^{\mathrm{op}}, E, s\right)$. As shown in [18], the pentagonal diffeomorphism $W$ is equal to (id $\left.\times m_{A}\right)\left(m_{B}^{T} \times\right.$ id) and the pentagon equation follows from the fact that $m_{B}^{T}$ is a morphism $G_{A} \rightarrow G_{A} \times G_{A}$. (Now it is clear that there are four multiplicative operators, since one can interchange $A$ and $B$; one can also consider $G_{A}^{\text {op }}$ and $G_{B}^{\text {op }}$.) But $W$ is not only a diffeomorphism but a diffeomorphism of a very special kind, namely, it is implemented by a bisection. Consider the set $U:=\left\{\left(g, s_{B}(g)\right): g \in G\right\}$. This is clearly a submanifold in $G \times G$. The following lemma is a result of direct computations.

Lemma 3.3. $U$ is a bisection of $G_{B}^{\mathrm{op}} \times G_{A}$, and $U \cdot(s, t)=W(s, t)$ (the left hand side is understood as the action of a bisection on a groupoid element). 
Because a bisection of a differential groupoid $\Gamma$ is an element of $M\left(C_{r}^{*}(\Gamma)\right)$ we conclude that $W \in M\left(C_{r}^{*}\left(G_{B}^{\mathrm{op}} \times G_{A}\right)\right)=M\left(C_{r}^{*}\left(G_{B}^{\mathrm{op}}\right) \otimes C_{r}^{*}\left(G_{A}\right)\right)$. The algebra $C_{r}^{*}\left(G_{B}^{\text {op }}\right)$ acts on $L^{2}\left(G_{B}^{\text {op }}\right)=L^{2}(G)$ in a non-degenerate way, therefore $W \in M\left(C B\left(L^{2}(G)\right) \otimes C_{r}^{*}\left(G_{A}\right)\right)$.

In what follows we will also need various modular functions related to DLG, so now we fix notation:

$$
\begin{array}{lll}
\psi_{A}(a)=\operatorname{det}\left(\left.P_{A} \operatorname{Ad}(a)\right|_{\mathfrak{a}}\right), & \psi_{B}(a)=\operatorname{det}\left(\left.P_{B} \operatorname{Ad}(a)\right|_{\mathfrak{b}}\right), & a \in A, \\
\varphi_{A}(b)=\operatorname{det}\left(\left.P_{A} \operatorname{Ad}(b)\right|_{\mathfrak{a}}\right), & \varphi_{B}(b)=\operatorname{det}\left(\left.P_{B} \operatorname{Ad}(b)\right|_{\mathfrak{b}}\right), & b \in B,
\end{array}
$$

where $P_{B}$ and $P_{A}$ denote the projections in $\mathfrak{g}$ corresponding to the decomposition $\mathfrak{g}=\mathfrak{a} \oplus \mathfrak{b}$.

The last object we define is a smooth function $Q: G \rightarrow \mathbb{R} \backslash\{0\}$. Let

$$
\operatorname{Ad}(g)=:\left(\begin{array}{ll}
g_{1} & g_{2} \\
g_{3} & g_{4}
\end{array}\right)
$$

be the decomposition of the adjoint representation with respect to the direct sum structure $\mathfrak{g}=\mathfrak{a} \oplus \mathfrak{b}$, i.e. $g_{1}: \mathfrak{a} \rightarrow \mathfrak{a}, g_{2}: \mathfrak{b} \rightarrow \mathfrak{a}$, etc. Then $Q$ is defined by

$$
G \ni g \mapsto Q(g):=\frac{\operatorname{det}(\operatorname{Ad}(g))}{\operatorname{det}\left(g_{1}\right) \operatorname{det}\left(g_{4}\right)} \in \mathbb{R} \backslash\{0\} .
$$

For $b \in B$ and $a \in A$ we have

$$
\operatorname{Ad}(a)=:\left(\begin{array}{ll}
\alpha_{1} & \alpha_{2} \\
0 & \alpha_{4}
\end{array}\right), \quad \operatorname{Ad}(b)=:\left(\begin{array}{ll}
\beta_{1} & 0 \\
\beta_{3} & \beta_{4}
\end{array}\right),
$$

and

$$
Q(b a)=\frac{\operatorname{det}\left(\beta_{4} \alpha_{4}\right)}{\operatorname{det}\left(\beta_{3} \alpha_{2}+\beta_{4} \alpha_{4}\right)} .
$$

The function $Q$ is related to the modular functions by the equalities

$$
Q(g)=\frac{\psi_{A}\left(a_{L}(g)\right) \varphi_{A}\left(b_{R}(g)\right)}{\psi_{A}\left(a_{R}(g)\right) \varphi_{A}\left(b_{L}(g)\right)}=\frac{\psi_{B}\left(a_{R}(g)\right) \varphi_{B}\left(b_{L}(g)\right)}{\psi_{B}\left(a_{L}(g)\right) \varphi_{B}\left(b_{R}(g)\right)} .
$$

The relationship between $Q$ and the groupoids $G_{A}$ and $G_{B}$ is described in the following lemma, the proof of which is straightforward.

LEMMA 3.4. $Q$ is a 1-cocycle on $G_{A}$ and $G_{B}$, i.e. $Q(a b) Q\left(b a^{\prime}\right)=Q\left(a b a^{\prime}\right)$, $Q(b a) Q\left(a b^{\prime}\right)=Q\left(b a b^{\prime}\right)$ for any $a, a^{\prime} \in A, b, b^{\prime} \in B$. Moreover, $Q$ is invariant with respect to the group inverse, i.e. $Q(g)=Q\left(g^{-1}\right)$.

The function $Q$ is exactly the one which appears in the definition of manageability of $W[16,12]$. For the geometric meaning of $Q$ see Appendix. define

Multiplication in $\mathcal{A}\left(G_{A}\right)$. Choose a real half-density $\mu_{0} \neq 0$ on $T_{e} B$ and

$$
\lambda_{0}(g)(v):=\mu_{0}\left(g^{-1} v\right), \quad v \in \Lambda^{\max } T_{g}^{l} G_{A} .
$$


It is easy to see that this is a left-invariant, non-vanishing half-density on $G_{A}$ and the corresponding right-invariant half-density is given by

$$
\varrho_{0}(g)(w)=\left|\psi_{B}\left(a_{L}(g)\right)\right|^{-1 / 2} \mu_{0}\left(w g^{-1}\right), \quad w \in \Lambda^{\max } T_{g}^{r} G_{A} .
$$

We put $\omega_{0}=\lambda_{0} \otimes \varrho_{0}$, and from (2) we obtain the expression for multiplication in $\mathcal{A}\left(G_{A}\right)$ :

$$
\left(\left(f_{1} \omega_{0}\right)\left(f_{2} \omega_{0}\right)\right)(g)=:\left(f_{1} * f_{2}\right)(g) \omega_{0}(g)
$$

where

$$
\begin{aligned}
& \left(f_{1} * f_{2}\right)(g)=\int_{B} \mu_{L}^{2}(b) f_{1}\left(a_{L}(g) b\right) f_{2}\left(b_{L}\left(a_{L}(g) b\right)^{-1} g\right) \\
& \quad=\int_{B} \mu_{R}^{2}(b)\left|\psi_{B}\left(a_{L}\left(b a_{R}(g)\right)\right)\right|^{-1} f_{1}\left(g b_{R}\left(b a_{R}(g)\right)^{-1}\right) f_{2}\left(b a_{R}(g)\right),
\end{aligned}
$$

and $\mu_{L}$ and $\mu_{R}$ are the left- and right-invariant half-densities on $B$ defined by $\mu_{0}$ (we use the fact that left and right fibers are diffeomorphic to $B$ ).

We finish this section with a simple observation, which will be used later on.

Lemma 3.5. For $g_{0} \in G$ the sets $\left\{g \in G:\left(g_{0}, g\right) \in \delta(G)\right\}=b_{R}\left(g_{0}\right) A$ and $\left\{g \in G:\left(g, g_{0}\right) \in \delta(G)\right\}=A b_{L}\left(g_{0}\right)$ are bisections of $G_{A}$.

4. Comultiplication. This section is entirely devoted to the proof that indeed $\widehat{\delta}$ can be used to obtain a comultiplication in the sense of the theory of quantum groups.

Theorem 4.1. Let $(G ; A, B)$ be a double Lie group, $G_{A}=\left(G, m_{A}, s_{A}, A\right)$ and $G_{B}=\left(G, m_{B}, s_{B}, B\right)$ the corresponding differential groupoids, and let $\delta:=m_{B}^{T}$.

(a) The mapping $\widehat{\delta}$ extends uniquely to $\Delta \in \operatorname{Mor}\left(C_{r}^{*}\left(G_{A}\right), C_{r}^{*}\left(G_{A}\right) \otimes\right.$ $\left.C_{r}^{*}\left(G_{A}\right)\right)$.

(b) $\Delta$ is coassociative, i.e. $(\Delta \otimes \mathrm{id}) \Delta=(\mathrm{id} \otimes \Delta) \Delta$.

(c) For any $a, b \in C_{r}^{*}\left(G_{A}\right)$, the elements $\Delta(a)(b \otimes I), \Delta(a)(I \otimes b)$ belong to $C_{r}^{*}\left(G_{A}\right) \otimes C_{r}^{*}\left(G_{A}\right)$. Moreover, the linear spaces $\operatorname{span}\{\Delta(a)(b \otimes I)$ : $\left.a, b \in C_{r}^{*}\left(G_{A}\right)\right\}$ and $\operatorname{span}\left\{\Delta(a)(I \otimes b): a, b \in C_{r}^{*}\left(G_{A}\right)\right\}$ are dense in $C_{r}^{*}\left(G_{A}\right) \otimes C_{r}^{*}\left(G_{A}\right)$.

In general we know [13] that $\widehat{\delta}$ extends to a morphism from $C^{*}\left(G_{A}\right)$ to $C^{*}\left(G_{A} \times G_{A}\right)$ and it is not a priori clear that it also defines a morphism of reduced algebras. However, it is easy to see that, for $\omega \in \mathcal{A}\left(G_{A}\right), \widehat{\delta}(\omega)$ defines a multiplier on $C_{r}^{*}\left(G_{A} \times G_{A}\right)$.

Indeed, let $\pi_{\text {id }}$ denote the identity representation of $\mathcal{A}\left(G_{A} \times G_{A}\right)$ on $L^{2}\left(G_{A} \times G_{A}\right)$. Proposition 1.5 yields $\left\|\pi_{\text {id }}\left(\widehat{\delta}(\omega) \omega_{1}\right)\right\|_{\widehat{\delta}}=\left\|\pi_{\delta}(\omega) \pi_{\mathrm{id}}\left(\omega_{1}\right)\right\| \leq$ $\left\|\pi_{\delta}(\omega)\right\|\left\|\pi_{\text {id }}\left(\omega_{1}\right)\right\|$. From this inequality we infer that $\widehat{\delta}(\omega)$ is a bounded, lin- 
ear mapping, defined on a dense, linear subspace of $C_{r}^{*}\left(G_{A} \times G_{A}\right)$. Therefore, it can be extended in a unique way to the whole $C_{r}^{*}\left(G_{A} \times G_{A}\right)$. Moreover, since $\omega_{2}^{*}\left(\widehat{\delta}(\omega) \omega_{1}\right)=\left(\widehat{\delta}\left(\omega^{*}\right) \omega_{2}\right)^{*} \omega_{1}$, we see that $\widehat{\delta}(\omega)$ is adjointable. Thanks to this property, $\widehat{\delta}(\omega)$ defines a multiplier.

Choose $\omega_{0}^{\prime} \in \mathcal{A}\left(G_{A} \times G_{A}\right)$ and let $\omega \in \mathcal{A}\left(G_{A} \times G_{A}\right)$. Take $\omega_{n} \in \mathcal{A}\left(G_{A}\right)$ as in Proposition 1.5(e). We have $\left\|\pi_{\mathrm{id}}\left(\widehat{\delta}\left(\omega_{n}\right) \omega\right)-\pi_{\mathrm{id}}(\omega)\right\| \leq\left\|\widehat{\delta}\left(\omega_{n}\right) \omega-\omega\right\|_{0}^{\prime}$; since this tends to 0 , we see that any element of $\mathcal{A}\left(G_{A} \times G_{A}\right)$ can be approximated in the norm defined by the identity representation by elements from $\widehat{\delta}\left(\mathcal{A}\left(G_{A}\right)\right) \mathcal{A}\left(G_{A} \times G_{A}\right)$. So the same is true for the whole $C_{r}^{*}\left(G_{A} \times G_{A}\right)$.

In this way to prove statement (a) of the theorem we need continuity of $\widehat{\delta}$ as a mapping defined on a dense subspace $\mathcal{A}\left(G_{A}\right) \subset C_{r}^{*}\left(G_{A}\right)$. This will follow immediately from

Proposition 4.2. Let $W$ be the pentagonal diffeomorphism defined in (6). The representation $\pi_{\delta}$ is implemented by $W$, i.e.

$$
\pi_{\delta}(\omega)=W\left(\pi_{\text {id }}(\omega) \otimes I\right) W^{*} .
$$

Proof. Clearly this result should be expected, taking into account the close relationship between the algebra defined by $W$ in a "standard way" and $C_{r}^{*}\left(G_{A}\right)$ (see Appendix B). We start by giving a formula for $\widehat{\delta}$. For $\omega=: f \omega_{0} \in \mathcal{A}\left(G_{A}\right)$ and $\omega_{1}=: F\left(\omega_{0} \otimes \omega_{0}\right) \in \mathcal{A}\left(G_{A} \times G_{A}\right)$, by (1) and Lemma 3.2 we have $\widehat{\delta}(\omega) \omega_{1}=\left(f *_{\delta} F\right)\left(\omega_{0} \otimes \omega_{0}\right)$, and

$$
\begin{aligned}
& \left(f *_{\delta} F\right)\left(a_{1} b_{1}, a_{2} b_{2}\right) \\
& =\int_{F_{l}\left(a_{1} a_{2}\right)} \lambda_{0}^{2}\left(a_{1} a_{2} b\right) f\left(a_{1} a_{2} b\right) t_{\delta}\left(a_{1} a_{2} b ; b_{L}^{-1}\left(a_{1} a_{2} b\right) a_{1} b_{1}, a_{R}\left(a_{2} b\right) b^{-1} b_{2}\right) \\
& \quad \times F\left(b_{L}^{-1}\left(a_{1} a_{2} b\right) a_{1} b_{1}, a_{R}\left(a_{2} b\right) b^{-1} b_{2}\right) .
\end{aligned}
$$

As proved in [13], for any morphism $h$ of differential groupoids, the function $t_{h}$ is right-invariant with respect to multiplication in the second groupoid, i.e. $t_{h}(x, y)=t_{h}\left(x, e_{L}(y)\right)$. In the next lemma we prove a formula for this function.

LEMMA 4.3. $t_{\delta}\left(b_{1} a_{2} a_{3} ; a_{2}, a_{3}\right)=\left|\varphi_{B}\left(b_{R}\left(b_{1} a_{2}\right)\right)\right|^{-1 / 2}$.

Proof. We use the notation and results of Proposition 1.3 and Lemma 3.2. Let $x:=b_{1} a_{2} a_{3}, y:=\left(a_{2}, a_{3}\right)$ and $z:=m_{\delta}(x, y)=\left(b_{1} a_{2}, b_{R}\left(b_{1} a_{2}\right) a_{3}\right)$. Let $W:=T_{(x, y)}\left(G_{A} \times_{\delta} F_{r}(y)\right)$ and $U:=\operatorname{ker} \pi_{2} \subset W$. Vectors from $U$ are represented by curves $u(t):=\left(b_{1}(t) a_{2} a_{3} ; a_{2}, a_{3}\right)$ with $b_{1}(0)=b_{1}$. It is easy to see that the subspace $V \subset W$ of vectors represented by the curves

$$
v(t):=\left(b_{1} a_{L}\left(b_{2}(t) a_{2}\right) a_{L}\left(b_{3}(t) a_{3}\right) ; b_{2}(t) a_{2}, b_{3}(t) a_{3}\right), \quad b_{2}(0)=b_{3}(0)=e,
$$

is complementary to $U$. So an isomorphism $i_{1}: \Omega_{R}^{1 / 2}(x) \otimes \Omega_{R}^{1 / 2}(y) \rightarrow \Omega^{1 / 2} W$ 
is given by

$$
i_{1}\left(\varrho_{0}(x) \otimes \varrho_{0}^{\prime}(y)\right)(u \wedge v):=\varrho_{0}(x)\left(\pi_{1} u\right) \varrho_{0}^{\prime}(y)\left(\pi_{2} v\right),
$$

where $u \in \Lambda^{\max } U, v \in \Lambda^{\max } V$.

Let $\widetilde{W}:=T_{(x, z)}\left(G_{A} *_{\delta} F_{r}(y)\right)$. Then $\widetilde{U}:=\operatorname{ker} \widetilde{\pi}_{2} \subset \widetilde{W}$ is the subspace of vectors represented by the curves $\widetilde{u}(t):=\left(b_{1} a_{2} a_{3} b_{1}(t) ; b_{1} a_{2}, b_{R}\left(b_{1} a_{2}\right) a_{3}\right)$ with $b_{1}(0)=e$. Choosing some $\widetilde{V} \subset \widetilde{W}$ complementary to $\widetilde{U}$ we can write an isomorphism $i_{2}: \Omega_{L}^{1 / 2}(x) \otimes \Omega_{R}^{1 / 2}(z) \rightarrow \Omega^{1 / 2} \widetilde{W}$ as

$$
i_{2}\left(\lambda_{0}(x) \otimes \varrho_{0}^{\prime}(z)\right)(\widetilde{u} \wedge \widetilde{v}):=\lambda_{0}(x)\left(\widetilde{\pi}_{1} \widetilde{u}\right) \varrho_{0}^{\prime}(z)\left(\widetilde{\pi}_{2} \widetilde{v}\right)
$$

for $\widetilde{u} \in \Lambda^{\max } \widetilde{U}$ and $\widetilde{v} \in \Lambda^{\max } \widetilde{V}$.

The function $t_{\delta}$ is defined by $\mathfrak{t}_{\delta} i_{1}\left(\varrho_{0}(x) \otimes \varrho_{0}^{\prime}(y)\right)=: t_{\delta}(x, y) i_{2}\left(\lambda_{0}(x) \otimes\right.$ $\left.\varrho_{0}^{\prime}(z)\right)$, i.e.

$$
i_{1}\left(\varrho_{0}(x) \otimes \varrho_{0}^{\prime}(y)\right)(u \wedge v)=t_{\delta}(x, y) i_{2}\left(\lambda_{0}(x) \otimes \varrho_{0}^{\prime}(z)\right)\left(\mathfrak{t}_{\delta}(u) \wedge \mathfrak{t}_{\delta}(v)\right) .
$$

Since the subspace $\mathfrak{t}_{\delta}(V)$ is represented by the curves

$$
t \mapsto\left(b_{1} a_{L}\left(b_{2}(t) a_{2}\right) a_{L}\left(b_{3}(t) a_{3}\right) ; b_{1} b_{2}(t) a_{2}, b_{R}\left(b_{1} a_{L}\left(b_{2}(t) a_{2}\right)\right) b_{3}(t) a_{3}\right),
$$

one immediately sees that it is complementary to $\widetilde{U}$. So to compute $t_{\delta}$ we need a decomposition of $\mathfrak{t}_{\delta}(u)$ with respect to the direct sum $\widetilde{W}=\widetilde{U} \oplus \mathfrak{t}_{\delta}(V)$. This is given by curves $\widetilde{u}(t), v(t)$ such that $\mathfrak{t}_{\delta} u(t)=\left(\mathfrak{t}_{d} v(t)\right)\left(x^{-1}, z^{-1}\right) \widetilde{u}(t)$ (group multiplication in $G \times G \times G$ ). Using our parametrization we see that for a given curve $b_{1}(t)$ with $b_{1}(0)=b_{1}$ we have to find curves $\widetilde{b}_{1}(t)$ with $\widetilde{b}_{1}(0)=e$ and $b_{2}(t), b_{3}(t)$ with $b_{2}(0)=b_{3}(0)=e$ such that

$$
\begin{aligned}
\left(b_{1}(t) a_{2} a_{3} ; b_{1}(t) a_{2}, b_{R}\left(b_{1}(t) a_{2}\right) a_{3}\right) & \\
= & \left(b_{1} a_{L}\left(b_{2}(t) a_{2}\right) a_{L}\left(b_{3}(t) a_{3}\right) ; b_{1} b_{2}(t) a_{2}, b_{R}\left(b_{1} a_{L}\left(b_{2}(t) a_{2}\right)\right) b_{3}(t) a_{3}\right) \\
& \cdot\left(b_{1} a_{2} a_{3} ; b_{1} a_{2}, b_{R}\left(b_{1} a_{2}\right) a_{3}\right)^{-1}\left(b_{1} a_{2} a_{3} \widetilde{b}_{1}(t) ; b_{1} a_{2}, b_{R}\left(b_{1} a_{2}\right) a_{3}\right) .
\end{aligned}
$$

The solution of this equation is given by

$$
\widetilde{b}_{1}(t)=b_{R}\left(b_{1}^{-1} b_{1}(t) a_{2} a_{3}\right), \quad b_{2}(t)=b_{1}^{-1} b_{1}(t), \quad b_{3}(t)=b_{R}\left(b_{1}^{-1} b_{1}(t) a_{2}\right) .
$$

Now we know enough to perform computations for the function $t_{\delta}$. Let $\left(X_{1}, \ldots, X_{n}\right)$ be a basis in $T_{e} B$ such that $\mu_{0}\left(X_{1} \wedge \cdots \wedge X_{n}\right)=1$. Then $\left(X_{1} x, \ldots, X_{n} x\right)$ is a basis in $T_{x}^{r} G_{A}$ and $u_{i}:=\left(X_{i} x ; 0_{a_{2}}, 0_{a_{3}}\right), i=1, \ldots, n$, form a basis in $U$. We also have the corresponding bases in $T_{a_{2}}^{r} G_{A}$ and $T_{a_{3}}^{r} G_{A}$, and $\left(v_{1}, \ldots, v_{n}, v_{1}^{\prime}, \ldots, v_{n}^{\prime}\right)$, where $v_{i}:=\left(b_{1} a_{L}\left(X_{i} a_{2}\right) a_{3} ; X_{i} a_{2}, 0_{a_{3}}\right)$ and $v_{i}^{\prime}:=\left(b_{1} a_{2} a_{L}\left(X_{i} a_{3}\right) ; 0_{a_{2}}, X_{i} a_{3}\right)$, is a basis in $V$. For $\varrho_{0}^{\prime}=\varrho_{0} \otimes \varrho_{0}, \varrho_{0}$ as in (12), equation (14) gives

$$
i_{1}\left(\varrho_{0}(x) \otimes \varrho_{0}^{\prime}(y)\right)\left(u_{1} \wedge \cdots \wedge v_{n}^{\prime}\right)=\left|\psi_{B}\left(a_{L}(x)\right) \psi_{B}\left(a_{2}\right) \psi_{B}\left(a_{3}\right)\right|^{-1 / 2} .
$$

Let $P$ be the projection onto $\widetilde{U}$ corresponding to the decomposition $\widetilde{W}=$ 
$\widetilde{U} \oplus \mathfrak{t}_{\delta}(V)$. Using (15) we can now rewrite (16) as

$$
\begin{aligned}
& \left|\psi_{B}\left(a_{L}(x)\right) \psi_{B}\left(a_{2}\right) \psi_{B}\left(a_{3}\right)\right|^{-1 / 2} \\
& \quad=t_{\delta}\left(x ; a_{2}, a_{3}\right) i_{2}\left(\lambda_{0}(x) \otimes \varrho_{0}^{\prime}(z)\right)\left(\mathfrak{t}_{\delta}\left(u_{1}\right) \wedge \cdots \wedge \mathfrak{t}_{\delta}\left(u_{n}\right) \wedge \cdots \wedge \mathfrak{t}_{\delta}\left(v_{n}^{\prime}\right)\right) \\
& \quad=t_{\delta}\left(x ; a_{2}, a_{3}\right) \lambda_{0}(x)\left(\widetilde{\pi}_{1} P \mathfrak{t}_{\delta} u_{1} \wedge \cdots \wedge \widetilde{\pi}_{1} P \mathfrak{t}_{d} u_{1}\right) \varrho_{0}^{\prime}(z)\left(\widetilde{\pi}_{2} \mathfrak{t}_{\delta} v_{1} \wedge \cdots \wedge \widetilde{\pi}_{2} \mathfrak{t}_{\delta} v_{n}^{\prime}\right) .
\end{aligned}
$$

From (17) we get

$$
\begin{gathered}
\tilde{\pi}_{1} P u_{i}=x b_{R}\left(x^{-1} X_{i} x\right), \quad \tilde{\pi}_{2} \mathfrak{t}_{\delta} v_{i}=\left(b_{1} X_{i} a_{2}, b_{R}\left(b_{1} a_{L}\left(X_{i} a_{2}\right)\right) a_{3}\right), \\
\tilde{\pi}_{2} \mathfrak{t}_{\delta} v_{i}^{\prime}=\left(0_{b_{1} a_{2}}, b_{R}\left(b_{1} a_{2}\right) X_{i} a_{3}\right),
\end{gathered}
$$

therefore

$$
\begin{aligned}
\lambda_{0}(x)\left(\widetilde{\pi}_{1} P \mathfrak{t}_{\delta} u_{1} \wedge \cdots \wedge\right. & \left.\widetilde{\pi}_{1} P \mathfrak{t}_{d} u_{1}\right) \varrho_{0}^{\prime}(z)\left(\widetilde{\pi}_{2} \mathfrak{t}_{\delta} v_{1} \wedge \cdots \wedge \widetilde{\pi}_{2} \mathfrak{t}_{\delta} v_{n}^{\prime}\right) \\
= & \left|\psi_{B}\left(a_{L}\left(b_{1} a_{2}\right)\right) \psi_{B}\left(a_{L}\left(b_{R}\left(b_{1} a_{2}\right) \widetilde{a}_{3}\right)\right)\right|^{-1 / 2} \\
& \times\left|\operatorname{det}\left(\left.P_{B} \operatorname{Ad}\left(x^{-1}\right)\right|_{\mathfrak{b}}\right)\right|^{1 / 2}\left|\operatorname{det}\left(\left.P_{B} \operatorname{Ad}\left(b_{1}\right)\right|_{\mathfrak{b}}\right)\right|^{1 / 2} \\
& \times\left|\operatorname{det}\left(\left.P_{B} \operatorname{Ad}\left(b_{R}\left(b_{1} a_{2}\right)\right)\right|_{\mathfrak{b}}\right)\right|^{1 / 2} .
\end{aligned}
$$

But since we have $\psi_{B}\left(a_{1} a_{2}\right)=\psi_{B}\left(a_{1}\right) \psi_{B}\left(a_{2}\right)$ and $a_{L}\left(b_{1} a_{2}\right) a_{L}\left(b_{R}\left(b_{1} a_{2}\right) a_{3}\right)=$ $a_{L}\left(b_{1} a_{2} a_{3}\right)=a_{L}(x)$, our equation for $t_{\delta}$ reduces to

$$
\begin{aligned}
& \left|\psi_{B}\left(a_{2} a_{3}\right)\right|^{-1 / 2} \\
& \quad=t_{\delta}\left(x ; a_{2}, a_{3}\right)\left|\operatorname{det}\left(\left.P_{B} \operatorname{Ad}\left(x^{-1}\right) P_{B} \operatorname{Ad}\left(b_{1}\right) P_{B} \operatorname{Ad}\left(b_{R}\left(b_{1} a_{2}\right)\right)\right|_{\mathfrak{b}}\right)\right|^{1 / 2} .
\end{aligned}
$$

Since $x=b_{1} a_{2} a_{3}$ we have

$$
\begin{aligned}
\left.P_{B} \operatorname{Ad}\left(x^{-1}\right) P_{B} \operatorname{Ad}\left(b_{1}\right) P_{B} \operatorname{Ad}\left(b_{R}\left(b_{1} a_{2}\right)\right)\right|_{\mathfrak{b}} & \\
& =\left.P_{B} \operatorname{Ad}\left(a_{3}^{-1} a_{2}^{-1}\right) P_{B} \operatorname{Ad}\left(b_{R}\left(b_{1} a_{2}\right)\right)\right|_{\mathfrak{b}},
\end{aligned}
$$

and finally we get $t_{\delta}\left(b_{1} a_{2} a_{3} ; a_{2}, a_{3}\right)=\left|\varphi_{B}\left(b_{R}\left(b_{1} a_{2}\right)\right)\right|^{-1 / 2}$.

In this way we arrive at a formula for $\widehat{\delta}$ (we recall that $f *_{\delta} F$ is defined by $\left.\widehat{\delta}\left(f \omega_{0}\right)\left(F\left(\omega_{0} \otimes \omega_{0}\right)\right)=:\left(f *_{\delta} F\right)\left(\omega_{0} \otimes \omega_{0}\right)\right)$ :

$$
\begin{aligned}
& \left(f *_{\delta} F\right)\left(a_{1} b_{1}, a_{2} b_{2}\right) \\
= & \int_{F_{l}\left(a_{1} a_{2}\right)} \lambda_{0}^{2}\left(a_{1} a_{2} b\right)\left|\varphi_{B}\left(b_{L}\left(a_{2} b\right)\right)\right|^{-1 / 2} f\left(a_{1} a_{2} b\right) \\
& \times F\left(b_{L}^{-1}\left(a_{1} a_{2} b\right) a_{1} b_{1}, a_{R}\left(a_{2} b\right) b^{-1} b_{2}\right) \\
= & \int_{B} \mu_{L}^{2}(b)\left|\varphi_{B}\left(b_{L}\left(a_{2} b\right)\right)\right|^{-1 / 2} f\left(a_{1} a_{2} b\right) F\left(b_{L}^{-1}\left(a_{1} a_{2} b\right) a_{1} b_{1}, a_{R}\left(a_{2} b\right) b^{-1} b_{2}\right) .
\end{aligned}
$$

Now we can prove the main formula of Proposition 4.2. Let $\varrho_{0}$ be the right-invariant half-density defined in (12). Choose a real half-density $\nu_{0}$ on $T_{e} A$ and let $\nu_{l}$ be the corresponding left-invariant half-density on $A$. Then $\psi_{0}:=\varrho_{0} \otimes \nu_{l}$ is a non-vanishing half-density on $G$. Define $\Psi_{0}:=\psi_{0} \otimes \psi_{0}$; 
this is a non-vanishing half-density on $G \times G$. An explicit formula for $\Psi_{0}$ is

$$
\begin{aligned}
\Psi_{0}(s, t)(Y & \left.s \wedge s \wedge Y_{1} t \wedge t X_{1}\right) \\
= & \varrho_{0}(s)(Y s) \varrho_{0}(t)\left(Y_{1} t\right) \nu_{l}\left(a_{R}(s)\right)\left(a_{R}(s) X\right) \nu_{l}\left(a_{R}(t)\right)\left(a_{R}(t) X_{1}\right) \\
=\left|\psi_{B}\left(a_{L}(s) a_{L}(t)\right)\right|^{-1 / 2} \mu_{0}(Y) \mu_{0}\left(Y_{1}\right) \nu_{0}(X) \nu_{0}\left(X_{1}\right), & \\
& Y, Y_{1} \in \Lambda^{\max _{0}} T_{e} B, X, X_{1} \in \Lambda^{\max } T_{e} A .
\end{aligned}
$$

In the next lemma we compute the action of $W$ on $\Psi_{0}$.

Lemma 4.4. Let $(s, t) \in G \times G,(\widetilde{s}, \widetilde{t}):=W^{-1}(s, t), X, X_{1} \in \Lambda^{\max } T_{e} A$ and $Y, Y_{1} \in \Lambda^{\max } T_{e} B$. Then

$$
\varphi_{B}\left(b_{R}(s)\right) \psi_{A}\left(a_{L}(\widetilde{t})\right) W^{-1}\left(Y s \wedge s X \wedge Y_{1} t \wedge t X_{1}\right)=Y \widetilde{s} \wedge \widetilde{s} X \wedge Y_{1} \tilde{t} \wedge \tilde{t} X_{1} .
$$

Proof. For $(s, t) \in G \times G$ let $i_{s t}: \mathfrak{b} \oplus \mathfrak{a} \oplus \mathfrak{b} \oplus \mathfrak{a} \rightarrow T_{(s, t)}(G \times G)$ be the isomorphism given by the decomposition $T_{(s, t)}(G \times G)=\mathfrak{b} s \oplus s \mathfrak{a} \oplus \mathfrak{b} t \oplus t \mathfrak{a}$. Then

$$
W^{-1}\left(Y s \wedge s X \wedge Y_{1} t \wedge t X_{1}\right)=\operatorname{det}\left(i_{\widetilde{s} \tilde{t}}^{-1} T_{(s, t)}\left(W^{-1}\right) i_{s t}\right)\left(Y \widetilde{s} \wedge \widetilde{s} X \wedge Y_{1} \widetilde{t} \wedge \widetilde{t} X_{1}\right)
$$

Set $F:=i_{\widetilde{s} \tilde{t}}^{-1} T_{(s, t)}\left(W^{-1}\right) i_{s t}$ and write $F$ in a block form corresponding to the decomposition $T_{(e, e)}(G \times G)=\mathfrak{b} \oplus \mathfrak{a} \oplus \mathfrak{b} \oplus \mathfrak{a}$ :

$$
F=\left(\begin{array}{llll}
F_{11} & F_{12} & F_{13} & F_{14} \\
F_{21} & F_{22} & F_{23} & F_{24} \\
F_{31} & F_{32} & F_{33} & F_{34} \\
F_{41} & F_{42} & F_{43} & F_{44}
\end{array}\right) .
$$

From the formula for $W^{-1}$ given in (6) we easily obtain

$$
F_{11}=I=F_{44} \quad \text { and } \quad F_{12}=F_{13}=F_{14}=F_{41}=F_{42}=F_{43}=0,
$$

so $\operatorname{det} F=\operatorname{det}\left(\begin{array}{ll}F_{22} & F_{23} \\ F_{32} & F_{33}\end{array}\right)$, and the mappings $F_{22}, F_{23}, F_{32}, F_{33}$ are given by:

$$
\begin{aligned}
F_{22} & : \mathfrak{a} \ni X \mapsto \operatorname{Ad}\left(a_{L}(z)^{-1}\right) X-P_{A} \operatorname{Ad}\left(b_{R}(z) a_{L}(t)^{-1}\right) P_{B} \operatorname{Ad}\left(b_{R}(s)^{-1}\right) X, \\
F_{23} & : \mathfrak{b} \ni Y \mapsto P_{A} \operatorname{Ad}\left(b_{R}(z) a_{L}(t)^{-1}\right) Y, \\
F_{32} & : \mathfrak{a} \ni X \mapsto-\operatorname{Ad}\left(b_{R}(s)^{-1}\right) P_{B} \operatorname{Ad}\left(b_{R}(s)^{-1}\right) X, \\
F_{33} & : \mathfrak{b} \ni Y \mapsto \operatorname{Ad}\left(b_{R}(s)^{-1}\right) Y,
\end{aligned}
$$

where $z:=b_{R}(s)^{-1} a_{L}(t)$. Now it remains to compute the determinant and compare it with the definitions of $\varphi_{B}$ and $\psi_{A}$.

Having these results we are ready to prove Proposition 4.2. Let $w$ : $G \times G \rightarrow \mathbb{R}$ be defined by $W \Psi_{0}=: w \Psi_{0}$ and $W(F)$ by $W\left(F \Psi_{0}\right)=: W(F) \Psi_{0}$. Then $W(F)(s, t)=F\left(W^{-1}(s, t)\right) w(s, t)$. From the preceding lemma we get

$$
w(s, t)=\left|\varphi_{B}\left(b_{R}(s)\right) \psi_{A}\left(a_{L}(\widetilde{t})\right) \psi_{B}\left(a_{L}(\widetilde{t})\right)\right|^{-1 / 2} \quad \text { where } \quad \tilde{t}=b_{R}(s)^{-1} t .
$$


Now let us compute $\pi_{\delta}(\omega) W$. For $\omega=: f \omega_{0}$ and $\Psi=: F \Psi_{0}$ using (18) we get

$$
\begin{aligned}
\left(f *_{\delta}\right. & W(F))\left(a_{1} b_{1}, a_{2} b_{2}\right) \\
= & \int_{B} \mu_{L}^{2}(b)\left|\varphi_{B}\left(b_{L}\left(a_{2} b\right)\right)\right|^{-1 / 2} f\left(a_{1} a_{2} b\right) W(F)\left(b_{L}^{-1}\left(a_{1} a_{2} b\right) a_{1} b_{1}, a_{R}\left(a_{2} b\right) b^{-1} b_{2}\right) \\
= & \int_{B} \mu_{L}^{2}(b)\left|\varphi_{B}\left(b_{L}\left(a_{2} b\right)\right)\right|^{-1 / 2} f\left(a_{1} a_{2} b\right) w\left(b_{L}^{-1}\left(a_{1} a_{2} b\right) a_{1} b_{1}, a_{R}\left(a_{2} b\right) b^{-1} b_{2}\right) \\
& \times F\left(b_{L}^{-1}\left(a_{1} a_{2} b\right) a_{1} a_{2} b_{L}\left(a_{2}^{-1} b_{1}\right), b_{1}^{-1} a_{2} b_{2}\right) \\
= & \left|\psi_{A}\left(a_{L}\left(b_{1}^{-1} a_{2}\right)\right) \psi_{B}\left(a_{L}\left(b_{1}^{-1} a_{2}\right)\right)\right|^{-1 / 2} \\
& \times \int_{B} \mu_{L}^{2}(b)\left|\varphi_{B}\left(b_{L}\left(a_{2} b\right)\right) \varphi_{B}\left(b_{L}^{-1}\left(a_{2} b\right) b_{1}\right)\right|^{-1 / 2} f\left(a_{1} a_{2} b\right) \\
& \times F\left(b_{L}^{-1}\left(a_{1} a_{2} b\right) a_{1} a_{2} b_{L}\left(a_{2}^{-1} b_{1}\right), b_{1}^{-1} a_{2} b_{2}\right) \\
= & \left|\psi_{A}\left(a_{L}\left(b_{1}^{-1} a_{2}\right)\right) \psi_{B}\left(a_{L}\left(b_{1}^{-1} a_{2}\right)\right) \varphi_{B}\left(b_{1}\right)\right|^{-1 / 2} \\
& \times \int_{B} \mu_{L}^{2}(b) f\left(a_{1} a_{2} b\right) F\left(b_{L}^{-1}\left(a_{1} a_{2} b\right) a_{1} a_{2} b_{L}\left(a_{2}^{-1} b_{1}\right), b_{1}^{-1} a_{2} b_{2}\right) .
\end{aligned}
$$

On the other hand, using the formula (13) for multiplication and Lemma 2.3 we can write

$$
\begin{gathered}
W((f \otimes I) * F)\left(a_{1} b_{1}, a_{2} b_{2}\right)=w\left(a_{1} b_{1}, a_{2} b_{2}\right)((f \otimes I) * F)\left(a_{1} b_{1} a_{L}\left(b_{1}^{-1} a_{2}\right), b_{1}^{-1} a_{2} b_{2}\right) \\
=\left|\psi_{A}\left(a_{L}\left(b_{1}^{-1} a_{2}\right)\right) \psi_{B}\left(a_{L}\left(b_{1}^{-1} a_{2}\right)\right) \varphi_{B}\left(b_{1}\right)\right|^{-1 / 2} \\
\quad \times \int_{B} \mu_{L}^{2}(b) f\left(a_{1} a_{2} b\right) F\left(b_{L}^{-1}\left(a_{1} a_{2} b\right) a_{1} a_{2} b_{L}\left(a_{2}^{-1} b_{1}\right), b_{1}^{-1} a_{2} b_{2}\right) .
\end{gathered}
$$

Comparing both expressions we see that $\pi_{\delta}(\omega) W=W\left(\pi_{\text {id }}(\omega) \otimes I\right)$. Proposition 4.2 is proved.

In this way the proof of statement (a) of Theorem 4.1 has been completed, i.e. $\widehat{\delta}$ can be extended to a morphism $\Delta$ from $C_{r}^{*}\left(G_{A}\right)$ to $C_{r}^{*}\left(G_{A}\right) \otimes$ $C_{r}^{*}\left(G_{A}\right)$.

(b) Proposition 4.2 immediately yields coassociativity of $\Delta$ by a standard proof based on the pentagonal equation.

(c) Density conditions. Our proof of the density conditions will be the following. First, note that, by using standard density arguments, one easily shows that if $\widehat{\delta}(\omega)\left(\omega_{1} \otimes I\right) \in \mathcal{A}\left(G_{A} \times G_{A}\right)$ then also $\Delta(a)(b \otimes I) \in C_{r}^{*}\left(G_{A}\right) \otimes$ $C_{r}^{*}\left(G_{A}\right)$ for any $a, b \in C_{r}^{*}\left(G_{A}\right)$, and the same holds for the second inclusion.

We begin by giving explicit formulae for $\widehat{\delta}(\omega)\left(I \otimes \omega_{2}\right)$ and $\widehat{\delta}(\omega)\left(\omega_{1} \otimes I\right)$ for $\omega, \omega_{1}, \omega_{2} \in \mathcal{A}\left(G_{A}\right)$ based on some geometric considerations. These objects will be elements of $\mathcal{A}\left(G_{A} \times G_{A}\right)$. Next, we verify that our guess is correct. Finally, we check the density conditions. 
Let us first give some heuristic arguments for our formulae. Let us write $\widehat{\delta}(\omega)=\sum \omega_{k}^{\prime} \otimes \omega_{k}^{\prime \prime}$ and formally compute

$$
\begin{aligned}
\widehat{\delta}(\omega)\left(I \otimes \omega_{1}\right)\left(g_{1}, g_{2}\right) & =\sum \omega_{k}^{\prime}\left(g_{1}\right)\left(\omega_{k}^{\prime \prime} \omega_{1}\right)\left(g_{2}\right) \\
& =\left[\left(\sum \omega_{k}^{\prime} \otimes \omega_{k}^{\prime \prime}\right)\left(g_{1}, \cdot\right) \omega_{1}\right]\left(g_{2}\right)=\left[\widehat{\delta}(\omega)\left(g_{1}, \cdot\right) \omega_{1}\right]\left(g_{2}\right) .
\end{aligned}
$$

What kind of object is $\widehat{\delta}(\omega)\left(g_{1}, \cdot\right)$ ? Since $\delta=m_{B}^{T}$, we can expect that $\widehat{\delta}(\omega)\left(g_{1}, \cdot\right)=\omega\left(m_{B}\left(g_{1}, \cdot\right)\right)$. Now using Lemma 3.5 , we can interpret $\omega\left(m_{B}\left(g_{1}, \cdot\right)\right)$ as a function on the bisection $b_{R}\left(g_{1}\right) A$, and this can be identified with a multiplier on $\mathcal{A}\left(G_{A}\right)$. Therefore, the natural candidate for $\widehat{\delta}(\omega)\left(I \otimes \omega_{1}\right)$ is $\omega\left(m_{B}\left(g_{1}, \cdot\right)\right) \omega_{1}$. It follows that (almost) the same is true in the differential case. Let $\delta^{R}$ and $\delta^{L}$ be the mappings associated to the morphism $\delta$ described in Lemma 3.2:

$$
\begin{array}{ll}
\delta^{L}\left(g_{1} ; a_{2}, a_{3}\right)=\left(a_{2} b_{L}\left(a_{3} b_{R}\left(g_{1}\right)\right), a_{3} b_{R}\left(g_{1}\right)\right), & a_{L}\left(g_{1}\right)=a_{2} a_{3}, \\
\delta^{R}\left(g_{1} ; a_{2}, a_{3}\right)=\left(b_{L}\left(g_{1}\right) a_{2}, b_{R}\left(b_{L}\left(g_{1}\right) a_{2}\right) a_{3}\right), & a_{R}\left(g_{1}\right)=a_{2} a_{3} .
\end{array}
$$

In the following lemma we denote the tangents to these mappings by the same letters.

Lemma 4.5. For any $\left(g_{1}, g_{2} ; g\right) \in \delta$ the mappings

and

$$
\delta^{L}: T_{g}^{l} G_{A} \rightarrow T_{\left(g_{1}, g_{2}\right)}^{l}\left(G_{A} \times G_{A}\right)=T_{g_{1}}^{l} G_{A} \oplus T_{g_{2}}^{l} G_{A}
$$

$$
\delta^{R}: T_{g}^{r} G_{A} \rightarrow T_{\left(g_{1}, g_{2}\right)}^{r}\left(G_{A} \times G_{A}\right)=T_{g_{1}}^{r} G_{A} \oplus T_{g_{2}}^{r} G_{A}
$$

are injective. Moreover, the mappings $\pi_{1} \delta^{L}: T_{g}^{l} G_{A} \rightarrow T_{g_{1}}^{l} G_{A}, \pi_{1} \delta^{R}: T_{g}^{r} G_{A} \rightarrow$ $T_{g_{1}}^{r} G_{A}, \pi_{2} \delta^{L}: T_{g}^{l} G_{A} \rightarrow T_{g_{2}}^{l} G_{A}, \pi_{2} \delta^{R}: T_{g}^{r} G_{A} \rightarrow T_{g_{2}}^{r} G_{A}$, where $\pi_{1}, \pi_{2}$ are the projections from $G_{A} \times G_{A}$ to $G_{A}$, are isomorphisms.

Proof. Let $g_{1}:=a_{1} b_{1}=\widetilde{b}_{1} \widetilde{a}_{1}, g_{2}:=b_{1} \widetilde{a}_{2}=a_{2} b_{2}, g:=\delta^{T}\left(g_{1}, g_{2}\right)=$ $a_{1} b_{1} \widetilde{a}_{2}=\widetilde{b}_{1} \widetilde{a}_{1} \widetilde{a}_{2}=a_{1} a_{2} b_{2}$. Let $X \in T_{g}^{l} G_{A}$ and $Y \in T_{g}^{r} G_{A}$ be represented by curves $a_{1} a_{2} b_{2}(t)$ with $b_{2}(0)=b_{2}$ and $\widetilde{b}_{1}(t) \widetilde{a}_{1} \widetilde{a}_{2}$ with $\widetilde{b}_{1}(0)=\widetilde{b}_{1}$ respectively. Then $\delta^{L}(X)$ and $\delta^{R}(Y)$ are represented by $\left(a_{1} b_{L}\left(a_{2} b_{2}(t)\right), a_{2} b_{2}(t)\right)$ and $\left(\widetilde{b}_{1}(t) \widetilde{a}_{1}, b_{R}\left(\widetilde{b}_{1}(t) \widetilde{a}_{1}\right) \widetilde{a}_{2}\right)$ and the first statement is clear. Direct computations also prove that $\left(\pi_{1} \delta^{L}\right)^{-1},\left(\pi_{2} \delta^{L}\right)^{-1},\left(\pi_{1} \delta^{R}\right)^{-1}$ and $\left(\pi_{2} \delta^{R}\right)^{-1}$ are given by:

$$
\begin{array}{lll}
\left(\pi_{1} \delta^{L}\right)^{-1}: a_{1} b_{1}(t) \mapsto a_{1} a_{2} b_{L}\left(a_{2}^{-1} b_{1}(t)\right), & b_{1}(0)=b_{1} ; \\
\left(\pi_{2} \delta^{L}\right)^{-1}: a_{2} b_{2}(t) \mapsto a_{1} a_{2} b_{2}(t), & & b_{2}(0)=b_{2} ; \\
\left(\pi_{1} \delta^{R}\right)^{-1}: \widetilde{b_{1}}(t) \widetilde{a_{1}} \mapsto \widetilde{b_{1}}(t) \widetilde{a_{1}} \widetilde{a_{2}}, & \widetilde{b_{1}}(0)=\widetilde{b}_{1} ; \\
\left(\pi_{2} \delta^{R}\right)^{-1}: \widetilde{b_{2}}(t) \widetilde{a_{2}} \mapsto b_{R}\left(\widetilde{b_{2}}(t) \widetilde{a_{1}}-1\right) \widetilde{a_{1}} \widetilde{a_{2}}, & \widetilde{b}_{2}(0)=b_{1} .
\end{array}
$$

Now suppose we are given $\omega \in \mathcal{A}\left(G_{A}\right), g_{1} \in G_{A}$ and $u_{1}:=v_{1} \otimes w_{1} \in$ $\Lambda^{\max } T_{g_{1}}^{l} G_{A} \otimes \Lambda^{\max } T_{g_{1}}^{r} G_{A}$. For each $g_{2}$ such that $\left(g_{1}, g_{2}\right) \in \delta\left(G_{A}\right)$ we can 
define the number

$$
\delta_{1}\left(\omega ; g_{1}, u_{1}\right)\left(g_{2}\right):=\omega\left(\delta^{T}\left(g_{1}, g_{2}\right)\right)\left(\left(\pi_{1} \delta^{L}\right)^{-1} v_{1} \otimes\left(\pi_{1} \delta^{R}\right)^{-1} w_{1}\right) .
$$

Since the set of possible $g_{2}$ is the bisection $b_{R}\left(g_{1}\right) A$, in this way we get a function on this bisection. In the same way for each $g_{2} \in G_{A}$ and $u_{2}:=$ $v_{2} \otimes w_{2} \in \Lambda^{\max } T_{g_{2}}^{l} G_{A} \otimes \Lambda^{\max } T_{g_{2}}^{r} G_{A}$ we get a function on the bisection $A b_{L}\left(g_{2}\right)$ given by

$$
\delta_{2}\left(\omega ; g_{2}, u_{2}\right)\left(g_{1}\right):=\omega\left(\delta^{T}\left(g_{1}, g_{2}\right)\right)\left(\left(\pi_{2} \delta^{L}\right)^{-1} v_{2} \otimes\left(\pi_{2} \delta^{R}\right)^{-1} w_{2}\right) .
$$

Having a bisection $U$ and a function $f$ on it, we can define a natural action of this pair on bidensities by the formula $((f U) \omega)(g):=f\left(g^{\prime}\right)(U \omega)(g)$, where $g^{\prime}$ is the unique point in $U$ such that $a_{L}\left(g^{\prime}\right)=a_{L}(g)$. Now for $\omega, \omega_{1}, \omega_{2} \in \mathcal{A}\left(G_{A}\right)$ we define $K_{1}\left(\omega, \omega_{1}\right) \in \mathcal{A}\left(G_{A} \times G_{A}\right)$ and $K_{2}\left(\omega, \omega_{2}\right) \in \mathcal{A}\left(G_{A} \times G_{A}\right)$ by

$$
\begin{aligned}
& K_{1}\left(\omega, \omega_{1}\right)\left(g_{1}, g_{2}\right)\left(u_{1} \otimes u_{2}\right):=\left[\delta_{1}\left(\omega ; g_{1}, u_{1}\right)\left(b_{R}\left(g_{1}\right) A\right) \omega_{1}\right]\left(g_{2}\right)\left(u_{2}\right), \\
& K_{2}\left(\omega, \omega_{2}\right)\left(g_{1}, g_{2}\right)\left(u_{1} \otimes u_{2}\right):=\left[\delta_{2}\left(\omega ; g_{2}, u_{2}\right)\left(A b_{L}\left(g_{2}\right)\right) \omega_{2}\right]\left(g_{1}\right)\left(u_{1}\right) .
\end{aligned}
$$

It is clear that these formulae are bilinear, so in fact $K_{1}, K_{2}: \mathcal{A}\left(G_{A}\right) \otimes$ $\mathcal{A}\left(G_{A}\right) \rightarrow \mathcal{A}\left(G_{A} \times G_{A}\right)$. These mappings are our candidates for $\widehat{\delta}(\omega)\left(I \otimes \omega_{1}\right)$ and $\widehat{\delta}(\omega)\left(\omega_{1} \otimes I\right)$. In the next lemma we compute the functions $\delta_{1}$ and $\delta_{2}$.

Lemma 4.6. Let $\left(g_{1}, g_{2}\right) \in \delta\left(G_{A}\right)$ and let $u_{1}, u_{2}$ be as above. Then

$$
\begin{aligned}
& \delta_{1}\left(f \omega_{0} ; g_{1}, u_{1}\right)\left(g_{2}\right)=f\left(\delta^{T}\left(g_{1}, g_{2}\right)\right)\left|\psi_{B}\left(a_{L}\left(g_{2}\right)\right)\right|^{-1 / 2}\left|\psi_{B}\left(a_{R}\left(g_{2}\right)\right)\right|^{-1 / 2} \omega_{0}\left(g_{1}\right)\left(u_{1}\right), \\
& \delta_{2}\left(f \omega_{0} ; g_{2}, u_{2}\right)\left(g_{1}\right)=f\left(\delta^{T}\left(g_{1}, g_{2}\right)\right) \omega_{0}\left(g_{2}\right)\left(u_{2}\right) .
\end{aligned}
$$

Proof. Let $g_{1}=a_{1} b_{1}, g_{2}=b_{1} \widetilde{a}_{2}, g:=\delta^{T}\left(g_{1}, g_{2}\right)=a_{1} b_{1} \widetilde{a}_{2}$ and $u_{1}=$ $v_{1} \otimes w_{1}$. Using the definitions of $\omega_{0}$ and $\delta_{1}$ we compute

$$
\begin{aligned}
\delta_{1}\left(\omega ; g_{1}, u_{1}\right)\left(g_{2}\right) & \\
& =\omega(g)\left(\left(\pi_{1} \delta^{L}\right)^{-1} v_{1} \otimes\left(\pi_{1} \delta^{R}\right)^{-1} w_{1}\right) \\
& =f(g) \omega_{0}\left(\left(\pi_{1} \delta^{L}\right)^{-1} v_{1} \otimes\left(\pi_{1} \delta^{R}\right)^{-1} w_{1}\right) \\
& =f(g) \mu_{0}\left(g^{-1}\left(\pi_{1} \delta^{L}\right)^{-1} v_{1}\right)\left|\psi_{B}\left(a_{L}(g)\right)\right|^{-1 / 2} \mu_{0}\left(\left(\pi_{1} \delta^{R}\right)^{-1} w_{1} g^{-1}\right) .
\end{aligned}
$$

Let us represent elements from $T_{g_{1}}^{l} G_{A}$ by $g_{1} X$ for $X \in T_{e} B$ and elements from $T_{g_{1}}^{r} G_{A}$ by $X g_{1}$ for $X \in T_{e} B$. Then from Lemma 4.5 we see that the mapping $T_{e} B \ni X \mapsto g^{-1}\left(\pi_{1} \delta^{L}\right)^{-1} g_{1} X \in T_{e} B$ is equal to $P_{B} \operatorname{Ad}\left(\widetilde{a}_{2}^{-1}\right)$, and $T_{e} B \ni X \mapsto\left(\pi_{1} \delta^{R}\right)^{-1} X g_{1} g^{-1} \in T_{e} B$ is the identity mapping. Since $a_{L}(g)=a_{L}\left(g_{1}\right) a_{L}\left(g_{2}\right)$ the first formula is proved. In the same way one can prove the second.

To find explicit expressions for $K_{1}$ and $K_{2}$ we also need formulae for the actions of the bisections $b A$ and $A b$ on $\mathcal{A}\left(G_{A}\right)$. 
Lemma 4.7. For $b_{0} \in B$ the actions of the bisections $b_{0} A$ and $A b_{0}$ on $\omega_{0}$ are given by

$$
\begin{aligned}
& \left(\left(b_{0} A\right) \omega_{0}\right)(g)=\left|\varphi_{B}\left(b_{0}\right)\right|^{-1 / 2}\left|\frac{\psi_{B}\left(a_{L}\left(b_{0}^{-1} g\right)\right)}{\psi_{B}\left(a_{L}(g)\right)}\right|^{-1 / 2} \omega_{0}(g), \\
& \left(\left(A b_{0}\right) \omega_{0}\right)(g)=\left|\varphi_{B}\left(b_{0}\right)\right|^{-1 / 2} \omega_{0}(g) .
\end{aligned}
$$

Proof. Let $\left(b_{0} A\right) \omega_{0}=: f \omega_{0}$ and $h:=\left(b_{0} A\right) \cdot g=b_{0} g$. Using (5) we get

$$
f(h)=\left|\frac{\psi_{B}\left(a_{L}(g)\right)}{\psi_{B}\left(a_{L}(h)\right)}\right|^{-1 / 2} \frac{\mu_{0}(w)}{\mu_{0}\left(\left(\left(b_{0} A\right) \cdot(w g)\right) h^{-1}\right)} \quad \text { for } w \in \Lambda^{\max } T_{e} B .
$$

Let $X \in T_{e} B$ be represented by a curve $b(t)$; then $\left(\left(b_{0} A\right) \cdot(X g)\right) h^{-1}$ is represented by $b_{0} b(t) b_{0}^{-1}$ and this gives us the first formula.

To prove the second one, again put $h:=\left(A b_{0}\right) \cdot g$ and $\left(A b_{0}\right) \omega_{0}=: f \omega_{0}$, but now $h=b_{L}\left(a_{L}(g) b_{0}^{-1}\right)^{-1} g=a_{R}\left(a_{L}(g) b_{0}^{-1}\right) b_{0} b_{R}(g)$ and $g=b_{L}\left(a_{L}(h) b_{0}\right)^{-1} h$ $=a_{R}\left(a_{L}(h) b_{0}\right) b_{0}^{-1} b_{R}(h)$. For $X$ represented by a curve $b(t)$, the vector $\left(\left(A b_{0}\right)\right.$. $(X g)) h^{-1}$ is represented by

$$
\widetilde{b}(t):=b_{L}\left(a_{L}(b(t) g) b_{0}^{-1}\right)^{-1} b(t) b_{L}\left(a_{L}(g) b_{0}^{-1}\right) .
$$

To simplify expressions write $g=: a b$. Now we observe that $\widetilde{b}(t)=: b_{1}(t) b_{2}(t)$ for $b_{1}(t):=b_{L}\left(a_{L}(b(t) a) b_{0}^{-1}\right)^{-1} b_{L}\left(a b_{0}^{-1}\right)$ with $b_{1}(0)=e$ and $b_{2}(t):=$ $b_{L}\left(a b_{0}^{-1}\right)^{-1} b(t) b_{L}\left(a b_{0}^{-1}\right)$ with $b_{2}(0)=e$. One easily verifies that

$$
b_{1}(t)=b_{R}\left[b_{R}\left(b_{0} a^{-1}\right) a a_{R}\left(a^{-1} b(t)^{-1} a\right) a^{-1} b_{R}\left(b_{0} a^{-1}\right)^{-1}\right] .
$$

From this decomposition it follows that the tangent mapping is given by

$$
T_{e} B \ni X \mapsto \operatorname{Ad}\left(b_{R}\left(b_{0} a^{-1}\right)\right) X-P_{B} \operatorname{Ad}\left(b_{R}\left(b_{0} a^{-1}\right) a\right) P_{A} \operatorname{Ad}\left(a^{-1}\right) X .
$$

Let

$$
\operatorname{Ad}(a)=:\left(\begin{array}{cc}
\alpha_{1} & \alpha_{2} \\
0 & \alpha_{4}
\end{array}\right) \text { and } \operatorname{Ad}\left(b_{R}\left(b_{0} a^{-1}\right)\right)=:\left(\begin{array}{cc}
\beta_{1} & 0 \\
\beta_{3} & \beta_{4}
\end{array}\right)
$$

be the decompositions as in (9). With this notation, our mapping is equal to $\beta_{4}+\beta_{3} \alpha_{2} \alpha_{4}^{-1}$. Substituting it into the expression for $f$ and using the formula for $h$ one gets

$$
\begin{aligned}
f(h) & =\left|\frac{\psi_{B}\left(a_{L}(g)\right)}{\psi_{B}\left(a_{R}\left(a b_{0}^{-1}\right)\right)}\right|^{-1 / 2}\left|\operatorname{det}\left(\beta_{4}+\beta_{3} \alpha_{2} \alpha_{4}^{-1}\right)\right|^{-1 / 2} \\
& =\left|\frac{\operatorname{det}\left(\beta_{4} \alpha_{4}+\beta_{3} \alpha_{2}\right)}{\psi_{B}\left(a_{R}\left(a b_{0}^{-1}\right)\right)}\right|^{-1 / 2} .
\end{aligned}
$$

But since $b_{R}\left(b_{0} a^{-1}\right) a=a_{R}\left(a b_{0}^{-1}\right) b_{0}$, we get $f(h)=\left|\varphi_{B}\left(b_{0}\right)\right|^{-1 / 2}$. 
Now, using Lemmas 4.7 and 4.6 , we easily derive the formulae for the mappings $K_{1}$ and $K_{2}$ (we write $K_{i}\left(f \omega_{0}, f_{i} \omega_{0}\right)=: K_{i}\left(f, f_{i}\right)\left(\omega_{0} \otimes \omega_{0}\right)$ ):

$$
\begin{aligned}
& K_{1}\left(f, f_{1}\right)\left(a_{1} b_{1}, a_{2} b_{2}\right) \\
& \quad=\left|\psi_{B}\left(a_{L}\left(b_{1}^{-1} a_{2}\right)\right)\right|^{-1}\left|\varphi_{B}\left(b_{1}\right)\right|^{-1 / 2} f\left(a_{1} b_{1} a_{L}\left(b_{1}^{-1} a_{2}\right)\right) f_{1}\left(b_{1}^{-1} a_{2} b_{2}\right), \\
& K_{2}\left(f, f_{2}\right)\left(a_{1} b_{1}, a_{2} b_{2}\right) \\
& \quad=\left|\varphi_{B}\left(b_{L}\left(g_{2}\right)\right)\right|^{-1 / 2} f\left(a_{1} a_{2} b_{2}\right) f_{2}\left(b_{L}^{-1}\left(a_{1} b_{L}\left(g_{2}\right)\right) a_{1} b_{1}\right) .
\end{aligned}
$$

LEMMA 4.8. $K_{1}\left(\omega, \omega_{1}\right)\left(\omega_{2} \otimes I\right)=\widehat{\delta}(\omega)\left(\omega_{2} \otimes \omega_{1}\right)=K_{2}\left(\omega, \omega_{2}\right)\left(I \otimes \omega_{1}\right)$.

Proof. Using (21) and Lemma 2.3 we compute

$$
\begin{aligned}
K_{1}\left(f, f_{1}\right) *\left(f_{2} \otimes I\right) & \left(a_{1} b_{1}, a_{2} b_{2}\right) \\
= & \int_{B} \mu_{L}^{2}(b)\left|\varphi_{B}(b)\right|^{-1 / 2}\left|\psi_{B}\left(a_{L}\left(b^{-1} a_{2}\right)\right)\right|^{-1} \\
& \quad \times f\left(a_{1} b a_{L}\left(b^{-1} a_{2}\right)\right) f_{2}\left(b_{L}^{-1}\left(a_{1} b\right) a_{1} b_{1}\right) f_{1}\left(b^{-1} a_{2} b_{2}\right) .
\end{aligned}
$$

The mapping $B \ni b \mapsto b_{L}\left(a_{2}^{-1} b\right) \in B$ is a diffeomorphism. Applying it to the integrated density one gets exactly (18). The second equality is immediate from Lemma 2.3.

Now we know enough to get rid of $K_{1}$ and $K_{2}$, i.e. identify them with $\widehat{\delta}(\omega)\left(I \otimes \omega_{1}\right)$ and $\widehat{\delta}(\omega)\left(\omega_{1} \otimes I\right)$.

Proposition 4.9. Let $\omega=f \omega_{0}, \omega_{1}=f_{1} \omega_{0} \in \mathcal{A}\left(G_{A}\right)$. Then $\widehat{\delta}(\omega)\left(I \otimes \omega_{1}\right)$, $\widehat{\delta}(\omega)\left(\omega_{1} \otimes I\right) \in \mathcal{A}\left(G_{A} \times G_{A}\right)$. Moreover, for $f *_{\delta}\left(I \otimes f_{1}\right)$ and $f *_{\delta}\left(f_{1} \otimes I\right)$ given by $\widehat{\delta}\left(f \omega_{0}\right)\left(I \otimes f_{1} \omega_{0}\right)=:\left(f *_{\delta}\left(I \otimes f_{1}\right)\right)\left(\omega_{0} \otimes \omega_{0}\right)$ and $\widehat{\delta}\left(f \omega_{0}\right)\left(f_{1} \omega_{0} \otimes I\right)=$ : $\left(f *_{\delta}\left(f_{1} \otimes I\right)\right)\left(\omega_{0} \otimes \omega_{0}\right)$ we have the following expressions:

$$
\begin{aligned}
&\left(f *_{\delta}\left(I \otimes f_{1}\right)\right)\left(g_{1}, g_{2}\right)=\left|\psi_{B}\left(a_{L}\left(b_{R}\left(g_{1}\right)^{-1} g_{2}\right)\right)\right|^{-1}\left|\varphi_{B}\left(b_{R}\left(g_{1}\right)\right)\right|^{-1 / 2} \\
& \times f\left(g_{1} a_{L}\left(b_{R}\left(g_{1}\right)^{-1} g_{2}\right)\right) f_{1}\left(b_{R}\left(g_{1}\right)^{-1} g_{2}\right), \\
&\left(f *_{\delta}\left(f_{1} \otimes I\right)\right)\left(g_{1}, g_{2}\right)=\left|\varphi_{B}\left(b_{L}\left(g_{2}\right)\right)\right|^{-1 / 2} f\left(a_{L}\left(g_{1}\right) g_{2}\right) f_{1}\left(b_{L}\left(a_{L}\left(g_{1}\right) g_{2}\right)^{-1} g_{1}\right) .
\end{aligned}
$$

Proof. This proposition simply states that $\widehat{\delta}(\omega)\left(I \otimes \omega_{1}\right)=K_{1}\left(\omega, \omega_{1}\right)$ and $\widehat{\delta}(\omega)\left(\omega_{1} \otimes I\right)=K_{2}\left(\omega, \omega_{1}\right)$. Let us verify the first equality. We know that $K_{1}\left(\omega, \omega_{1}\right), K_{2}\left(\omega, \omega_{1}\right)$ and $\widehat{\delta}(\omega)$ are multipliers on $C_{r}^{*}\left(G_{A} \times G_{A}\right)$. Using Lemma 4.8 we have the following sequence of equalities for any $\omega, \omega_{1}, \omega_{2}, \omega_{3}$ in $\mathcal{A}\left(G_{A}\right)$ :

$$
\begin{aligned}
K_{1}\left(\omega, \omega_{1}\right)\left(\omega_{2} \otimes \omega_{3}\right) & \\
& =K_{1}\left(\omega, \omega_{1}\right)\left(\omega_{2} \otimes I\right)\left(I \otimes \omega_{3}\right)=\widehat{\delta}(\omega)\left(\omega_{2} \otimes \omega_{1}\right)\left(I \otimes \omega_{3}\right) \\
& =\widehat{\delta}(\omega)\left(I \otimes \omega_{1}\right)\left(\omega_{2} \otimes I\right)\left(I \otimes \omega_{3}\right)=\widehat{\delta}(\omega)\left(I \otimes \omega_{1}\right)\left(\omega_{2} \otimes \omega_{3}\right) .
\end{aligned}
$$

Therefore, the multipliers $K_{1}\left(\omega, \omega_{1}\right)$ and $\widehat{\delta}(\omega)\left(I \otimes \omega_{1}\right)$ agree on a dense subspace $\mathcal{A}\left(G_{A}\right) \otimes \mathcal{A}\left(G_{A}\right) \subset C_{r}^{*}\left(G_{A}\right) \otimes C_{r}^{*}\left(G_{A}\right)$, so they must be equal. The second equality can be proved in the same way. 
In this way the first part of statement (c) of Theorem 4.1 is proved.

Now we will prove the density conditions. Comparing the formula for $K_{1}\left(f, f_{1}\right)$ with the expression for $W$ given in $(6)$ we see that

$$
K_{1}\left(f, f_{1}\right)(s, t)=k_{1}(s, t)\left(f \otimes f_{1}\right)\left(W^{-1}(s, t)\right),
$$

where

$$
k_{1}(s, t):=\left|\psi_{B}\left(a_{L}\left(b_{R}(s)^{-1} t\right)\right)\right|^{-1}\left|\varphi_{B}\left(b_{R}(s)\right)\right|^{-1 / 2} .
$$

Because $k_{1}(s, t) \neq 0$, we can apply Lemma 2.4 to conclude that elements in $\mathcal{A}\left(G_{A} \times G_{A}\right)$ can be approximated by elements of the form $K_{1}\left(\omega, \omega_{1}\right)$ in the norm given by $\omega_{0} \otimes \omega_{0}$. Then from Proposition $1.5(\mathrm{~d})$ they can be approximated also in $C_{r}^{*}\left(G_{A} \times G_{A}\right)=C_{r}^{*}\left(G_{A}\right) \otimes C_{r}^{*}\left(G_{A}\right)$, and since $\mathcal{A}\left(G_{A} \times G_{A}\right)$ is dense in $C_{r}^{*}\left(G_{A} \times G_{A}\right)$ we arrive at the conclusion that $\operatorname{span}\left\{\Delta(a)(I \otimes b): a, b \in C_{r}^{*}\left(G_{A}\right)\right\}$ is dense in $C_{r}^{*}\left(G_{A}\right) \otimes C_{r}^{*}\left(G_{A}\right)$.

The same argument can be applied to $K_{2}\left(f, f_{1}\right)$ since one easily checks that the mapping

$$
G \times G \ni(s, t) \mapsto\left(a_{L}(s) t, b_{L}\left(a_{L}(s) t\right)^{-1} s\right) \in G \times G
$$

is a diffeomorphism of $G \times G$ with the inverse

$$
\left.(s, t) \mapsto\left(b_{L}(s) t, a_{L}\left(b_{L}(s) t\right)^{-1}\right) s\right) .
$$

This completes the proof of the theorem.

In this way we have the main component of a quantum group structure on $C_{r}^{*}\left(G_{A}\right)$ and in the next section we will look for the other ones.

5. Hopf-like structure of $\mathcal{A}\left(G_{A}\right)$. In this section we identify other mappings which appear in the theory of quantum groups and show that the structure of the algebra $\mathcal{A}\left(G_{A}\right)$ is very similar to the structure of a Hopf algebra. Of course, because $\widehat{\delta}$ is not a mapping from $\mathcal{A}\left(G_{A}\right)$ to $\mathcal{A}\left(G_{A}\right) \otimes$ $\mathcal{A}\left(G_{A}\right)$ and even not to multipliers of $\mathcal{A}\left(G_{A}\right) \otimes \mathcal{A}\left(G_{A}\right)$ (in the sense of van Daele [3]), the pair $\left(\mathcal{A}\left(G_{A}\right), \widehat{\delta}\right)$ is not a true Hopf algebra.

"Coinverse". Let $Q$ be the function described in (9) of Section 3. For $z \in \mathbb{C}$ we define a mapping $\tau_{z}$ by

$$
\tau_{z}: \mathcal{A}\left(G_{A}\right) \ni \omega \mapsto|Q|^{i z} \omega \in \mathcal{A}\left(G_{A}\right)
$$

Let us also define a mapping $R: \mathcal{A}\left(G_{A}\right) \rightarrow \mathcal{A}\left(G_{A}\right)$ by

$$
R(\omega)(g)(v \otimes w):=\omega\left(g^{-1}\right)\left(w^{-1} \otimes v^{-1}\right)
$$

where $v \in \Lambda^{\max } T_{g}^{l} G_{A}, w \in \Lambda^{\max } T_{g}^{r} G_{A}$ and $v^{-1}, w^{-1}$ denote the images of $v, w$ under the mapping $g \mapsto g^{-1}$.

Defining $R f$ by $(R f) \omega_{0}:=R\left(f \omega_{0}\right)$ we easily get

$$
(R f)(g)=\left|\psi_{B}\left(a_{L}(g) a_{R}(g)\right)\right|^{1 / 2} f\left(g^{-1}\right) .
$$


Finally, if we define $\kappa: \mathcal{A}\left(G_{A}\right) \rightarrow \mathcal{A}\left(G_{A}\right)$ by

$$
\kappa:=R \cdot \tau_{i / 2} \quad \text { then } \quad(\kappa f)(g)=|Q(g)|^{-1 / 2}\left|\psi_{B}\left(a_{L}(g) a_{R}(g)\right)\right|^{1 / 2} f\left(g^{-1}\right) .
$$

It is clear that all these mappings are linear.

Proposition 5.1.

1. $\tau_{z}$ is a one-parameter (complex) group of automorphisms of $\mathcal{A}\left(G_{A}\right)$.

2. $\tau_{z} \cdot *=* \cdot \tau_{\bar{z}}$.

3. $R$ is an involutive *-antiautomorphism of $\mathcal{A}\left(G_{A}\right)$.

4. $R \cdot \tau_{z}=\tau_{z} \cdot R$.

5. $\kappa\left(\omega_{1} \omega_{2}\right)=\kappa\left(\omega_{2}\right) \kappa\left(\omega_{1}\right)$.

6. $\kappa \cdot * \cdot \kappa \cdot *=\mathrm{id}$.

Proof. Items 1 and 2 are immediate consequences of Lemma 3.4. They are true for any cocycle $Q$ on a differential groupoid [13].

3. Since $R$ is implemented by a group inverse, it is clear that $R$ is involutive. From the formula for $R f$ it follows immediately that $R$ commutes with the *-operation on $\mathcal{A}\left(G_{A}\right)$. To prove that $R$ is an antiautomorphism we compute:

$$
\begin{aligned}
R\left(f_{1} * f_{2}\right)(g) & =\left|\psi_{B}\left(a_{L}(g) a_{R}(g)\right)\right|^{1 / 2}\left(f_{1} * f_{2}\right)\left(g^{-1}\right) \\
& =\left|\psi_{B}(a \widetilde{a})\right|^{1 / 2} \int_{B} \mu_{L}^{2}\left(b^{\prime}\right) f_{1}\left(\widetilde{a}^{-1} b^{\prime}\right) f_{2}\left(b_{L}\left(\widetilde{a}^{-1} b^{\prime}\right)^{-1} g^{-1}\right),
\end{aligned}
$$

where $g=: a b=\widetilde{b} \widetilde{a}$. On the other hand,

$$
\begin{aligned}
\left(\left(R f_{2}\right) *\right. & \left.\left(R f_{1}\right)\right)(g)=\int_{B} \mu_{L}^{2}\left(b^{\prime}\right)\left(R f_{2}\right)\left(a b^{\prime}\right)\left(R f_{1}\right)\left(b_{L}\left(a b^{\prime}\right)^{-1} g\right) \\
& =\int_{B} \mu_{L}^{2}\left(b^{\prime}\right) f_{1}\left(g^{-1} b_{L}\left(a b^{\prime}\right)\right) f_{2}\left(b^{\prime-1} a^{-1}\right)\left|\psi_{B}\left(a a_{R}\left(a b^{\prime}\right)\right) \psi_{B}\left(a_{R}\left(a b^{\prime}\right) \widetilde{a}\right)\right|^{1 / 2} \\
& =\left|\psi_{B}(a \widetilde{a})\right|^{1 / 2} \int_{B} \mu_{L}^{2}\left(b^{\prime}\right)\left|\psi_{B}\left(a_{R}\left(a b^{\prime}\right)\right)\right| f_{1}\left(g^{-1} b_{L}\left(a b^{\prime}\right)\right) f_{2}\left(b^{\prime-1} a^{-1}\right) .
\end{aligned}
$$

Now apply the diffeomorphism $B \ni b^{\prime} \mapsto b_{L}\left(\widetilde{a} b^{-1} b^{\prime}\right) \in B$ to this integral to get the previous one.

4. This follows directly from the definitions of $R$ and $\tau$.

Items 5 and 6 are direct consequences of the definitions and the previous statements.

"Counit". Since $F_{l}(e)=F_{r}(e)=B$ (e is the neutral element in $G$ ), for any $b \in B$ there is a mapping $\Omega_{L}^{1 / 2}(b) \otimes \Omega_{R}^{1 / 2}(b) \ni \lambda \otimes \varrho \mapsto \lambda \varrho \in \Omega^{1}\left(T_{b} B\right)$. In this way restriction of $\omega \in \mathcal{A}\left(G_{A}\right)$ to $B$ defines a smooth 1-density on $B$ (with compact support). Integration of this density defines a linear functional $\varepsilon$ 
on $\mathcal{A}\left(G_{A}\right),\left.\mathcal{A}\left(G_{A}\right) \ni \omega \mapsto \int_{B} \omega\right|_{B} \in \mathbb{C}$. It is easy to see that

$$
\varepsilon\left(f \omega_{0}\right)=\int_{B} \mu_{L}^{2}(b)\left|\varphi_{B}(b)\right|^{1 / 2} f(b) .
$$

In the next proposition we denote by $\kappa \otimes \mathrm{id}$, id $\otimes \kappa, \varepsilon \otimes \mathrm{id}$, id $\otimes \varepsilon$ the natural extensions of these mappings to $\mathcal{A}\left(G_{A} \times G_{A}\right)$, and by $m: \mathcal{A}\left(G_{A} \times G_{A}\right) \rightarrow$ $\mathcal{A}\left(G_{A}\right)$ the natural extension of the multiplication map of $\mathcal{A}\left(G_{A}\right)$.

Proposition 5.2.

1. $\varepsilon$ is a character on $\mathcal{A}\left(G_{A}\right)$.

2. $(\varepsilon \otimes \mathrm{id}) \widehat{\delta}(\omega)\left(I \otimes \omega_{1}\right)=\omega \omega_{1}$ and $(\mathrm{id} \otimes \varepsilon)\left(\omega_{1} \otimes I\right) \widehat{\delta}(\omega)=\omega_{1} \omega$.

3. $m(\kappa \otimes \mathrm{id}) \widehat{\delta}(\omega)\left(I \otimes \omega_{1}\right)=\varepsilon(\omega) \omega_{1}$ and $m(\mathrm{id} \otimes \kappa)(\omega \otimes I) \widehat{\delta}\left(\omega_{1}\right)=\varepsilon\left(\omega_{1}\right) \omega$.

Proof. 1. The definition of $\varepsilon$ is a special case of the following one. Let $\Gamma$ be a differential groupoid and let $a \in E$ be such that $F_{l}(a)=F_{r}(a)$. Then we can define a linear functional $\varepsilon_{a}$ on $\mathcal{A}(\Gamma)$ by $\varepsilon_{a}(\omega):=\left.\int_{F_{l}(a)} \omega\right|_{F_{l}(a)}$. We will prove that $\varepsilon_{a}$ is a character on $\mathcal{A}(\Gamma)$. Let $\omega_{1}=f_{1} \omega_{0}$ and $\omega_{2}=f_{2} \omega_{0}$. Then

$$
\begin{aligned}
\varepsilon_{a}\left(\omega_{1} \omega_{2}\right) & =\int_{F_{l}(a)} \lambda_{0}(x) \varrho_{0}(x) \int_{F_{l}(a)} \lambda_{0}^{2}(y) f_{1}(y) f_{2}(s(y) x) \\
& =\int_{F_{l}(a) \times F_{l}(a)} \mu(x, y) f_{1}(y) f_{2}(s(y) x),
\end{aligned}
$$

where $\mu$ is a 1-density on $F_{l}(a) \times F_{l}(a)$ defined by $\mu(x, y)(v \otimes w):=\lambda_{0}(x)(v) \varrho_{0}(x)(v) \lambda_{0}^{2}(y)(w), \quad v \in \Lambda^{\max } T_{x}^{l} \Gamma, w \in \Lambda^{\max } T_{y}^{l} \Gamma$. Now consider a diffeomorphism $\Phi$ of $F_{l}(a) \times F_{l}(a)$ given by $(x, y) \mapsto(s(y) x, y)$. A short computation shows that $(\Phi \mu)(z, t)=\nu(z, t)$ for $\nu(z, t)(v \otimes w):=$ $\lambda_{0}(x)(v) \varrho_{0}(x)(v) \lambda_{0}(y)(w) \varrho_{0}(y)(w)$. In this way our integral is equal to

$$
\begin{aligned}
& \int_{F_{l}(a) \times F_{l}(a)} \nu(x, y) f_{1}(x) f_{2}(y) \\
& \quad=\left(\int_{F_{l}(a)} \lambda_{0}(x) \varrho_{0}(x) f_{1}(x)\right)\left(\int_{F_{l}(a)} \lambda_{0}(y) \varrho_{0}(y) f_{2}(y)\right)=\varepsilon_{a}\left(\omega_{1}\right) \varepsilon_{a}\left(\omega_{2}\right) .
\end{aligned}
$$

To prove the second property, notice that the density $\varrho_{0}(x) \lambda_{0}(x)$ is real and invariant with respect to $s: F_{l}(a) \rightarrow F_{l}(a)$. So we get

$$
\begin{aligned}
\varepsilon_{a}\left(\omega^{*}\right) & =\int_{F_{l}(a)} f^{*}(x) \lambda_{0}(x) \varrho_{0}(x)=\int_{F_{l}(a)} \overline{f(s(x))} \lambda_{0}(x) \varrho_{0}(x) \\
& =\int_{F_{l}(a)} \overline{f(x)} \lambda_{0}(x) \varrho_{0}(x)=\overline{\varepsilon_{a}(\omega)} .
\end{aligned}
$$


2. For $\omega=F\left(\omega_{0} \otimes \omega_{0}\right)$ we have

$$
(\varepsilon \otimes \mathrm{id})\left(F\left(\omega_{0} \otimes \omega_{0}\right)\right)(g)=\left(\int_{B} \mu_{L}^{2}(b)\left|\varphi_{B}(b)\right|^{1 / 2} F(b, g)\right) \omega_{0}(g) .
$$

Using Proposition 4.9 we compute

$$
\begin{aligned}
(\varepsilon \otimes \mathrm{id}) \widehat{\delta}\left(f \omega_{0}\right)(I & \left.\otimes f_{1} \omega_{0}\right)(g)=\left(\int_{B} \mu_{L}^{2}(b)\left|\varphi_{B}(b)\right|^{1 / 2}\left(f *_{\delta}\left(I \otimes f_{1}\right)\right)(b, g)\right) \omega_{0}(g) \\
& =\left(\int_{B} \mu_{L}^{2}(b)\left|\psi_{B}\left(a_{L}\left(b^{-1} g\right)\right)\right|^{-1} f\left(b a_{L}\left(b^{-1} g\right)\right) f_{1}\left(b^{-1} g\right)\right) \omega_{0}(g) .
\end{aligned}
$$

Now apply the diffeomorphism $B \ni b \mapsto b_{L}\left(a_{L}(g)^{-1} b\right) \in B$ to get the formula (13) for $\omega \omega_{1}$.

Since $\left(\omega_{1} \otimes I\right) \widehat{\delta}(\omega)=\left(\widehat{\delta}\left(\omega^{*}\right)\left(\omega_{1}^{*} \otimes I\right)\right)^{*}$, Proposition 4.9 easily yields $\left(f_{1} \omega_{0} \otimes I\right) \widehat{\delta}\left(f \omega_{0}\right)\left(g_{1}, g_{2}\right)$

$$
=\left|\varphi_{B}\left(b_{L}\left(g_{2}\right)\right)\right|^{1 / 2} f\left(a_{R}\left(a_{R}\left(g_{1}\right) b_{L}\left(g_{2}\right)^{-1}\right) g_{2}\right) f_{1}\left(g_{1} b_{L}\left(g_{2}\right)^{-1}\right)\left(\omega_{0} \otimes \omega_{0}\right)\left(g_{1}, g_{2}\right)
$$

and we compute

$$
\begin{array}{r}
(\mathrm{id} \otimes \varepsilon)\left(f_{1} \omega_{0} \otimes I\right) \widehat{\delta}\left(f \omega_{0}\right)(g)=\left(\int_{B} \mu_{L}^{2}(b)\left|\varphi_{B}(b)\right|^{1 / 2}\left(\left(f_{1} \otimes I\right) *_{\delta} f\right)(g, b)\right) \omega_{0}(g) \\
=\left(\int_{B} \mu_{L}^{2}(b)\left|\varphi_{B}(b)\right| f_{1}\left(g b^{-1}\right) f\left(a_{R}\left(a_{R}(g) b^{-1}\right) b\right)\right) \omega_{0}(g) .
\end{array}
$$

Again, applying the diffeomorphism $B \ni b \mapsto b_{R}(g) b^{-1} \in B$ we arrive at the formula (13) for $\omega_{1} \omega$.

3. For $F\left(\omega_{0} \otimes \omega_{0}\right) \in \mathcal{A}\left(G_{A} \times G_{A}\right)$ the mapping $m$ is given by (cf. (13)) $m\left(F\left(\omega_{0} \otimes \omega_{0}\right)\right)=(m F) \omega_{0}, \quad(m F)(g):=\int_{B} \mu_{L}^{2}(b) F\left(a_{L}(g) b, b_{L}\left(a_{L}(g) b\right)^{-1} g\right)$.

From the formula (25) for $\kappa$ and Proposition 4.9 we get

$(\kappa \otimes \mathrm{id}) \widehat{\delta}\left(f \omega_{0}\right)\left(I \otimes f_{1} \omega_{0}\right)\left(g_{1}, g_{2}\right)=(\kappa \otimes \mathrm{id})\left(f *_{\delta}\left(I \otimes f_{1}\right)\right)\left(g_{1}, g_{2}\right)\left(\omega_{0} \otimes \omega_{0}\right)\left(g_{1}, g_{2}\right)$, $(\kappa \otimes \mathrm{id})\left(f *_{\delta}\left(I \otimes f_{1}\right)\right)\left(g_{1}, g_{2}\right)$

$$
=\left|Q\left(g_{1}\right)\right|^{-1 / 2}\left|\psi_{B}\left(a_{L}\left(g_{1}\right) a_{R}\left(g_{1}\right)\right)\right|^{1 / 2}\left(f *_{\delta}\left(I \otimes f_{1}\right)\right)\left(g_{1}^{-1}, g_{2}\right) .
$$

Let $\underset{g}{g}=a b$. We need a value of this last function for $g_{1}=a \widetilde{b}$ and $g_{2}=$ $b_{L}(a \widetilde{b})^{-1} a b$. For these points we have $b_{R}\left(g_{1}^{-1}\right)=b_{L}(a \widetilde{b})^{-1}$ and

$$
\begin{aligned}
(\kappa \otimes \mathrm{id}) & \left(f *_{\delta}\left(I \otimes f_{1}\right)\right)\left(a \widetilde{b}, b_{L}(a \widetilde{b})^{-1} a b\right) \\
& =|Q(a \widetilde{b})|^{-1 / 2}\left|\psi_{B}\left(a a_{R}(a \widetilde{b})\right)\right|^{1 / 2}\left(f *_{\delta}\left(I \otimes f_{1}\right)\right)\left(\widetilde{b}^{-1} a^{-1}, b_{L}(a \widetilde{b})^{-1} a b\right) \\
& =|Q(a \widetilde{b})|^{-1 / 2}\left|\psi_{B}\left(a a_{R}(a \widetilde{b})\right)\right|^{1 / 2}\left|\psi_{B}(a)\right|^{-1}\left|\varphi_{B}\left(b_{L}(a \widetilde{b})\right)\right|^{1 / 2} f\left(\widetilde{b}^{-1}\right) f_{1}(a b) \\
& =\left|\varphi_{B}(\widetilde{b})\right|^{1 / 2} f\left(\widetilde{b}^{-1}\right) f_{1}(a b),
\end{aligned}
$$


where the last equality follows from (10). In this way

$$
m(\kappa \otimes \mathrm{id})\left(f *_{\delta}\left(I \otimes f_{1}\right)\right)(g)=f_{1}(g) \int_{B} \mu_{L}^{2}(b)\left|\varphi_{B}(b)\right|^{1 / 2} f\left(b^{-1}\right)=\varepsilon\left(f \omega_{0}\right) f_{1}(g) .
$$

The second equality can be proved by similar computations.

REMARK 5.3. The first two statements of the above proposition can also be proved by the following observation. If in a differential groupoid $\Gamma$ there is $a \in E$ such that $F_{l}(a)=F_{r}(a)$, then the relation $h_{a}: \Gamma \longrightarrow\{1\}$ defined by $\operatorname{Gr}\left(h_{a}\right):=\left\{(1, g): g \in F_{l}(a)\right\}$ is a morphism of differential groupoids. Since $\mathcal{A}(\{1\})=\mathbb{C}$ the associated mapping $\widehat{h}_{a}$ takes values in $\mathbb{C}$ and the functional $\varepsilon_{a}$ is just $\widehat{h}_{a}$. For the functional $\varepsilon, a=e$ (the neutral element) and the relation is $e_{B}^{T}\left(e_{B}\right.$ being the identity of $\left.G_{B}\right)$. So the second statement is a consequence of $\left(e_{B}^{T} \times \mathrm{id}\right) \delta=\left(\mathrm{id} \times e_{B}^{T}\right) \delta=\mathrm{id}$, and this is just the transposition of the equality $m_{B}\left(e_{B} \times \mathrm{id}\right)=m_{B}\left(\mathrm{id} \times e_{B}\right)=\mathrm{id}$.

The statements in the last proposition are just axioms for a Hopf algebra rewritten in a way that makes sense in our situation. Whether this need be formalized is a question of finding other interesting examples.

Now we describe how $\widehat{\delta}$ commutes with $R$ and $\tau_{z}$. Define a mapping $\sim: \mathcal{A}\left(G_{A} \times G_{A}\right) \rightarrow \mathcal{A}\left(G_{A} \times G_{A}\right)$ by $(\sim \omega)\left(g_{1}, g_{2}\right):=\omega\left(g_{2}, g_{1}\right)$.

Proposition 5.4. For any $z \in \mathbb{C}$ and $\omega, \omega_{1}, \omega_{2} \in \mathcal{A}\left(G_{A}\right)$,

$$
\begin{aligned}
\widehat{\delta}(R \omega)\left(R \omega_{1} \otimes R \omega_{2}\right) & =\sim(R \otimes R)\left(\left(\omega_{2} \otimes \omega_{1}\right) \widehat{\delta}(\omega)\right), \\
\widehat{\delta}\left(\tau_{z} \omega\right)\left(\omega_{1} \otimes \omega_{2}\right) & =\left(\tau_{z} \otimes \tau_{z}\right)\left(\widehat{\delta}(\omega)\left(\tau_{-z} \omega_{1} \otimes \tau_{-z} \omega_{2}\right)\right) .
\end{aligned}
$$

Proof. Let $\omega=: f \omega_{0}, \omega_{1}=: f_{1} \omega_{0}$ and $\omega_{2}=: f_{2} \omega_{0}$. Using the same notation as before, and the formulae for $\widehat{\delta}$ (see 18)) and $R$, we compute the left hand side of the first equality:

$$
\begin{aligned}
(R f) *_{\delta} & \left(R f_{1} \otimes R f_{2}\right)\left(a_{1} b_{1}, a_{2} b_{2}\right)=\int_{B} \mu_{L}^{2}(b)\left|\varphi_{B}\left(b_{L}\left(a_{2} b\right)\right)\right|^{-1 / 2} \\
& \times(R f)\left(a_{1} a_{2} b\right)\left(R f_{1}\right)\left(b_{L}^{-1}\left(a_{1} a_{2} b\right) a_{1} b_{1}\right)\left(R f_{2}\right)\left(a_{R}\left(a_{2} b\right) b^{-1} b_{2}\right) \\
= & \int_{B} \mu_{L}^{2}(b)\left|\varphi_{B}\left(b_{L}\left(a_{2} b\right)\right)\right|^{-1 / 2}\left|\psi_{B}\left(a_{1} a_{2} a_{R}\left(a_{1} a_{2} b\right)\right)\right|^{1 / 2} f\left(b^{-1} a_{2}^{-1} a_{1}^{-1}\right) \\
& \times\left|\psi_{B}\left(a_{R}\left(a_{1} a_{2} b\right) a_{L}\left(b^{-1} a_{2}^{-1}\right) a_{R}\left(a_{1} b_{1}\right)\right)\right|^{1 / 2} f_{1}\left(b_{1}^{-1} a_{1}^{-1} b_{L}\left(a_{1} a_{2} b\right)\right) \\
& \times\left|\psi_{B}\left(a_{R}\left(a_{2} b\right) a_{R}\left(a_{2} b_{2}\right)\right)\right|^{1 / 2} f_{2}\left(b_{2}^{-1} b a_{R}\left(a_{2} b\right)^{-1}\right) \\
= & \int_{B} \mu_{L}^{2}(b)\left|\varphi_{B}\left(b_{L}\left(a_{2} b\right)\right)\right|^{-1 / 2}\left|\psi_{B}\left(a_{1} a_{2} a_{R}\left(a_{1} a_{2} b\right)^{2} a_{R}\left(a_{1} b_{1}\right) a_{R}\left(a_{2} b_{2}\right)\right)\right|^{1 / 2} \\
& \times f\left(b^{-1} a_{2}^{-1} a_{1}^{-1}\right) f_{1}\left(b_{1}^{-1} a_{1}^{-1} b_{L}\left(a_{1} a_{2} b\right)\right) f_{2}\left(b_{2}^{-1} b a_{R}\left(a_{2} b\right)^{-1}\right)
\end{aligned}
$$




$$
\begin{aligned}
= & \left|\psi_{B}\left(a_{1} a_{2} a_{R}\left(a_{1} b_{1}\right) a_{R}\left(a_{2} b_{2}\right)\right)\right|^{1 / 2} \\
& \times \int_{B} \mu_{L}^{2}(b)\left|\varphi_{B}\left(b_{L}\left(a_{2} b\right)\right)\right|^{-1 / 2}\left|\psi_{B}\left(a_{R}\left(a_{1} a_{2} b\right)\right)\right| \\
& \times f\left(b^{-1} a_{2}^{-1} a_{1}^{-1}\right) f_{1}\left(b_{1}^{-1} a_{1}^{-1} b_{L}\left(a_{1} a_{2} b\right)\right) f_{2}\left(b_{2}^{-1} b a_{R}\left(a_{2} b\right)^{-1}\right) .
\end{aligned}
$$

And the right hand side is

$$
\begin{aligned}
\sim & (R \otimes R)\left(\left(f_{2} \otimes f_{1}\right) *_{\delta} f\right)\left(a_{1} b_{1}, a_{2} b_{2}\right)=(R \otimes R)\left(\left(f_{2} \otimes f_{1}\right) *_{\delta} f\right)\left(a_{2} b_{2}, a_{1} b_{1}\right) \\
= & \left.\left|\psi_{B}\left(a_{2} a_{1} a_{R}\left(a_{2} b_{2}\right) a_{R}\left(a_{1} b_{1}\right)\right)\right|^{1 / 2}\left(f_{2} \otimes f_{1}\right) *_{\delta} f\right)\left(b_{2}^{-1} a_{2}^{-1}, b_{1}^{-1} a_{1}^{-1}\right) \\
= & \left|\psi_{B}\left(a_{2} a_{1} a_{R}\left(a_{2} b_{2}\right) a_{R}\left(a_{1} b_{1}\right)\right)\right|^{1 / 2} \overline{\left(f^{*} *_{\delta}\left(f_{2}^{*} \otimes f_{1}^{*}\right)\right)\left(s_{B}\left(a_{2} b_{2}\right), s_{B}\left(a_{1} b_{1}\right)\right)} \\
= & \left|\psi_{B}\left(a_{2} a_{1} a_{R}\left(a_{2} b_{2}\right) a_{R}\left(a_{1} b_{1}\right)\right)\right|^{1 / 2} \overline{\left(f^{*} *_{\delta}\left(f_{2}^{*} \otimes f_{1}^{*}\right)\right)\left(a_{2}^{-1} b_{L}\left(a_{2} b_{2}\right), a_{1}^{-1} b_{L}\left(a_{1} b_{1}\right)\right)} \\
= & \left|\psi_{B}\left(a_{1} a_{2} a_{R}\left(a_{1} b_{1}\right) a_{R}\left(a_{2} b_{2}\right)\right)\right|^{1 / 2} \overline{\int \mu_{L}^{2}(b)\left|\varphi_{B}\left(b_{L}\left(a_{1}^{-1} b\right)\right)\right|^{-1 / 2}} \\
& \times \overline{f^{*}\left(a_{2}^{-1} a_{1}^{-1} b\right) f_{2}^{*}\left(b_{L}^{-1}\left(a_{2}^{-1} a_{1}^{-1} b\right) a_{2}^{-1} b_{L}\left(a_{2} b_{2}\right)\right) f_{1}^{*}\left(a_{R}\left(a_{1}^{-1} b\right) b^{-1} b_{L}\left(a_{1} b_{1}\right)\right)} \\
= & \left|\psi_{B}\left(a_{1} a_{2} a_{R}\left(a_{1} b_{1}\right) a_{R}\left(a_{2} b_{2}\right)\right)\right|^{1 / 2} \\
& \times \int_{B} \mu_{L}^{2}(b)\left|\varphi_{B}\left(b_{L}\left(a_{1}^{-1} b\right)\right)\right|^{-1 / 2} f\left(b_{L}\left(a_{2}^{-1} a_{1}^{-1} b\right)^{-1} a_{2}^{-1} a_{1}^{-1}\right) \\
& \times f_{1}\left(b_{1}^{-1} a_{1}^{-1} b\right) f_{2}\left(b_{2}^{-1} a_{2}^{-1} b_{R}\left(b^{-1} a_{1}\right)^{-1}\right) .
\end{aligned}
$$

Now use the diffeomorphism $b \mapsto b_{L}\left(a_{2}^{-1} a_{1}^{-1} b\right)$ to get equality of the two integrals.

To prove the second equality one immediately verifies that it is enough to prove that

$$
Q\left(a_{1} a_{2} b\right)=\frac{Q\left(a_{1} b_{1}\right) Q\left(a_{2} b_{2}\right)}{Q\left(b_{L}\left(a_{1} a_{2} b\right)^{-1} a_{1} b_{1}\right) Q\left(a_{R}\left(a_{2} b\right) b^{-1} b_{2}\right)} .
$$

But since $Q$ is a cocycle on $G_{A}$ and $G_{B}$, we have the equalities $Q\left(a_{1} a_{2} b\right)=$ $Q\left(a_{1} b_{L}\left(a_{2} b\right)\right) Q\left(a_{2} b\right), Q\left(b_{L}\left(a_{1} a_{2} b\right)^{-1} a_{1} b_{1}\right)=Q\left(b_{L}\left(a_{1} a_{2} b\right)^{-1} a_{1}\right) Q\left(a_{1} b_{1}\right)$ and $Q\left(a_{1} b_{L}\left(a_{2} b\right)\right)=Q\left(b_{L}\left(a_{1} a_{2} b\right)^{-1} a_{1}\right)^{-1}$. Using them we easily arrive at the desired result.

6. Haar measure. This section is devoted to the construction of a Haar weight on $C_{r}^{*}\left(G_{A}\right)$. We refer to [14] and [10] for a detailed explanation what a Haar weight is on a locally compact quantum group. Let us also note that, in contrast to the theory of locally compact groups, the existence of a Haar weight is (up to now) contained in the definition of a (locally compact) quantum group. (It can be proved only for the compact case.) 
Let us begin by recalling some results from [13]. Let $(\Gamma, m, s, E)$ be a differential groupoid. Choose a real, non-vanishing, right-invariant halfdensity $\widetilde{\varrho}_{0}$. Let $\widetilde{\omega}_{0}=\widetilde{\lambda}_{0} \otimes \widetilde{\varrho}_{0}$ be the corresponding bidensity. Also choose a real, non-vanishing half-density $\nu$ on $E$. For such data we define:

- A smooth function $\sigma: \Gamma \rightarrow \mathbb{R} \backslash\{0\}$ by $\sigma(g)\left(\widetilde{\varrho}_{0} \otimes \nu\right)(g)=\left(\widetilde{\lambda}_{0} \otimes \nu\right)(g)$. We call $\sigma$ the modular function associated with the pair $\left(\widetilde{\varrho}_{0}, \nu\right)$. This function is a 1-cocycle on $\Gamma$ (i.e. $\sigma\left(g_{1} g_{2}\right)=\sigma\left(g_{1}\right) \sigma\left(g_{2}\right)$ for any composable $\left.g_{1}, g_{2} \in \Gamma\right)$. Therefore, it defines a one-parameter (complex) group of algebra automorphisms (though not $*$-automorphisms!) of $\mathcal{A}(\Gamma): \sigma_{z}(\omega)(g):=|\sigma(g)|^{2 i z} \omega(g)$.

- A positive linear functional $h$ on $\mathcal{A}(\Gamma)$ by $h\left(f \widetilde{\omega}_{0}\right):=\int_{E} \nu^{2} f$.

- A linear mapping $\widehat{h}: \mathcal{A}(\Gamma) \ni f \widetilde{\omega}_{0} \mapsto f \widetilde{\varrho}_{0} \otimes \nu \in L^{2}(\Gamma)$.

The next proposition describes the basic properties of these objects.

Proposition 6.1 ([13]). For any $\omega, \omega_{1} \in \mathcal{A}(\Gamma)$ :

- $h\left(\omega^{*} \omega\right)=(\widehat{h}(\omega) \mid \widehat{h}(\omega))$ (scalar product in $\left.L^{2}(\Gamma)\right)$.

- $h\left(\sigma_{z}(\omega) \omega_{1}\right)=h\left(\omega_{1} \sigma_{z-i}(\omega)\right)$.

- $\widehat{h}\left(\omega \omega_{1}\right)=\pi_{\text {id }}(\omega) \widehat{h}\left(\omega_{1}\right)$.

Now we specify the choice of $\widetilde{\varrho}_{0}$ and $\nu$ for our groupoid $G_{A}$. Let $\mu_{0} \neq 0$ be a real half-density on $T_{e} B$ and define a right-invariant half-density $\widetilde{\varrho}_{0}$ on $G_{A}$ by the formula

$$
\widetilde{\varrho}_{0}(g)(w):=\mu_{0}\left(w g^{-1}\right), \quad w \in \Lambda^{\max } T_{g}^{r} G_{A} .
$$

Let $\widetilde{\lambda}_{0}$ be the corresponding left-invariant half-density and $\widetilde{\omega}_{0}=\widetilde{\lambda}_{0} \otimes \widetilde{\varrho}_{0}$. A short computation shows that $\widetilde{\omega}_{0}(g)=\left|\psi_{B}\left(a_{L}(g) a_{R}(g)\right)\right|^{1 / 2} \omega_{0}$ (where $\omega_{0}$ denotes our standard bidensity constructed from $\mu_{0}$ as in (11), (12)). Also choose a real half-density $\nu_{0} \neq 0$ on $T_{e} A$ and let $\nu_{r}$ be the corresponding right-invariant half-density on $A$. In our standard representation of bidensities we get

$$
h\left(f \omega_{0}\right)=\int_{A} \nu_{r}^{2}(a)\left|\psi_{B}(a)\right|^{-1} f(a) .
$$

From the definitions of $h, \sigma$ and $\tau$ it is clear that $h \tau_{z}=h$ and $\sigma_{z} \tau_{w}=\tau_{w} \sigma_{z}$. In the next lemma we give a formula for the associated modular function.

Lemma 6.2. Let $\widetilde{\varrho}_{0}$ and $\nu_{r}$ be as above. Then the modular function $\sigma$ is given by

$$
\sigma(g)=\left|\frac{\psi_{A}\left(a_{L}(g)\right) \varphi_{A}\left(b_{R}(g)\right)}{\varphi_{B}\left(b_{L}(g)\right) \psi_{A}\left(a_{R}(g)\right)}\right|^{1 / 2} .
$$

Proof. From the definition of $\widetilde{\lambda}_{0}$ one can easily see that

$$
\tilde{\lambda}_{0}(g)(g v)=\left|\psi_{B}\left(a_{R}(g)\right)\right|^{1 / 2} \mu_{0}(v), \quad v \in \Lambda^{\max } T_{e} B .
$$


The subspace $g T_{e} A \subset T_{g} G$ is complementary to $T_{g}^{r} G_{A}$ and $T_{g}^{l} G_{A}$, i.e. $T_{g} G=$ $g T_{e} A \oplus T_{g}^{r} G_{A}=g T_{e} A \oplus T_{g}^{l} G_{A}$. Therefore, for $u \in \Lambda^{\max } T_{e} A, v \in \Lambda^{\max } T_{e} B$ we have the equalities

$\left(\widetilde{\varrho}_{0} \otimes \nu_{r}\right)(g)(v g \wedge g u)=\mu_{0}(v) \nu_{r}\left(a_{R}(g)\right)\left(a_{R}(g u)\right)=\left|\psi_{A}\left(a_{R}(g)\right)\right|^{1 / 2} \mu_{0}(v) \nu_{0}(u)$, $\left(\widetilde{\lambda}_{0} \otimes \nu_{r}\right)(g)(g v \wedge g u)=\left|\psi_{B}\left(a_{R}(g)\right)\right|^{1 / 2} \mu_{0}(v) \nu_{r}\left(a_{L}(g)\right)\left(a_{L}(g u)\right)$

$$
=\left|\psi_{B}\left(a_{R}(g)\right)\right|^{1 / 2}\left|\varphi_{A}\left(b_{R}(g)\right)\right|^{1 / 2}\left|\psi_{A}\left(a_{L}(g)\right)\right|^{1 / 2} \mu_{0}(v) \nu_{0}(u) .
$$

Now, since $v g \wedge g u=g\left(g^{-1} v g\right) \wedge g u=\operatorname{det}\left(\left.P_{B} \operatorname{Ad}\left(g^{-1}\right)\right|_{\mathfrak{b}}\right)(g v \wedge g u)$, we get the expression for $\sigma(g)$ :

$$
\begin{aligned}
\sigma(g)= & \left|\psi_{B}\left(a_{R}(g)\right)\right|^{1 / 2}\left|\varphi_{A}\left(b_{R}(g)\right)\right|^{1 / 2}\left|\psi_{A}\left(a_{L}(g)\right)\right|^{1 / 2} \\
& \times\left|\operatorname{det}\left(\left.P_{B} \operatorname{Ad}\left(g^{-1}\right)\right|_{\mathfrak{b}}\right)\right|^{1 / 2}\left|\psi_{A}\left(a_{R}(g)\right)\right|^{-1 / 2},
\end{aligned}
$$

and the equality $\operatorname{det}\left(\left.P_{B} \operatorname{Ad}\left(g^{-1}\right)\right|_{\mathfrak{b}}\right)=\psi_{B}\left(a_{R}(g)\right)^{-1} \varphi_{B}\left(b_{L}(g)\right)^{-1}$ implies the result.

Let $h \otimes \mathrm{id}: \mathcal{A}\left(G_{A} \times G_{A}\right) \rightarrow \mathcal{A}\left(G_{A}\right)$ be the natural extension of $h \otimes$ id : $\mathcal{A}\left(G_{A}\right) \otimes \mathcal{A}\left(G_{A}\right) \rightarrow \mathcal{A}\left(G_{A}\right)$, i.e.

$$
\left((h \otimes \mathrm{id})\left(F\left(\omega_{0} \otimes \omega_{0}\right)\right)\right)(g):=\left(\int_{A}\left|\psi_{B}(a)\right|^{-1} \nu_{r}^{2}(a) F(a, g)\right) \omega_{0}(g) .
$$

The next proposition states that the functional $h$ is "right-invariant", relates $h$ to $\kappa$, and describes the commutation of $\widehat{\delta}$ with the modular group $\sigma_{z}$. Recall that a functional $\varphi$ on a Hopf algebra $(\mathcal{A}, \Delta)$ is right-invariant iff $(\varphi \otimes \mathrm{id}) \Delta(a)=\varphi(a) I$.

Proposition 6.3. For any $\omega, \omega_{1}, \omega_{2} \in \mathcal{A}\left(G_{A}\right)$ :

$$
\begin{aligned}
(h \otimes \mathrm{id})\left(\widehat{\delta}\left(\omega_{1}\right)\left(I \otimes \omega_{2}\right)\right) & =h\left(\omega_{1}\right) \omega_{2}, \\
(h \otimes \mathrm{id})\left(\widehat{\delta}\left(\omega_{1}^{*}\right)\left(\omega_{2} \otimes I\right)\right) & =\kappa\left((h \otimes \mathrm{id})\left(\left(\omega_{1}^{*} \otimes I\right) \widehat{\delta}\left(\omega_{2}\right)\right)\right), \\
\widehat{\delta}\left(\sigma_{z}(\omega)\right)\left(\omega_{1} \otimes \omega_{2}\right) & =\left(\sigma_{z} \otimes \tau_{z}\right)\left(\widehat{\delta}(\omega)\left(\sigma_{-z} \omega_{1} \otimes \tau_{-z} \omega_{2}\right)\right) .
\end{aligned}
$$

Proof. Using Proposition 4.9 and right-invariance of $\nu_{r}$ we get

$$
\begin{aligned}
(h \otimes \mathrm{id})(\widehat{\delta} & \left.\left(f_{1} \omega_{0}\right)\left(I \otimes f_{2} \omega_{0}\right)\right)(g) \\
& =\left(\int_{A} \nu_{r}^{2}(a)\left|\psi_{B}(a)\right|^{-1}\left|\psi_{B}\left(a_{L}(g)\right)\right|^{-1} f_{1}\left(a a_{L}(g)\right) f_{2}(g)\right) \omega_{0}(g) \\
& =\left(\int_{A} \nu_{r}^{2}(a)\left|\psi_{B}\left(a a_{L}(g)\right)\right|^{-1} f_{1}\left(a a_{L}(g)\right)\right) \omega_{2}(g)=h\left(\omega_{1}\right) \omega_{2}(g) .
\end{aligned}
$$

To prove the second formula, we again use Proposition 4.9, right-invariance of $\nu_{r}$, and the formula for $\left(\omega_{1}^{*} \otimes I\right) \widehat{\delta}\left(\omega_{2}\right)$ given in the proof of Proposition 5.2 to obtain the left hand side of $(30)$ : 


$$
\begin{aligned}
& (h \otimes \mathrm{id})\left[\widehat{\delta}\left(\left(f_{1} \omega_{0}\right)^{*}\right)\left(f_{2} \omega_{0} \otimes I\right)\right](g) \\
& =\left(\int_{A} \nu_{r}^{2}(a)\left|\psi_{B}(a)\right|^{-1}\left(f_{1}^{*} *_{\delta}\left(f_{2} \otimes I\right)\right)(a, g)\right) \omega_{0}(g) \\
& =\left(\left|\varphi_{B}\left(b_{L}(g)\right)\right|^{-1 / 2} \int_{A} \nu_{r}^{2}(a)\left|\psi_{B}(a)\right|^{-1} \overline{f_{1}\left(s_{A}(a g)\right)} f_{2}\left(b_{L}(a g)^{-1} a\right)\right) \omega_{0}(g) .
\end{aligned}
$$

And the right hand side of this equality is

$$
\begin{aligned}
\kappa\left((h \otimes \mathrm{id})\left(\left(\left(f_{1} \omega_{0}\right)^{*} \otimes I\right) \widehat{\delta}\left(f_{2} \omega_{0}\right)\right)\right)(g)=\left(|Q(g)|^{-1 / 2}\left|\psi_{B}\left(a_{L}(g) a_{R}(g)\right)\right|^{1 / 2}\right. \\
\left.\quad \times \int_{A} \nu_{r}^{2}(a)\left|\psi_{B}(a)\right|^{-1}\left(\left(f_{1}^{*} \otimes I\right) *_{\delta} f_{2}\right)\left(a, g^{-1}\right)\right) \omega_{0}(g) \\
=\left(|Q(g)|^{-1 / 2}\left|\psi_{B}\left(a_{L}(g) a_{R}(g)\right)\right|^{1 / 2}\left|\varphi_{B}\left(g^{-1}\right)\right|^{1 / 2}\right. \\
\quad \times \int_{A} \nu_{r}^{2}(a)\left|\psi_{B}(a)\right|^{-1} \frac{f_{1}\left(s_{A}\left(a b_{R}(g)\right)\right)}{\left.f_{2}\left(a_{R}\left(a b_{R}(g)\right) g^{-1}\right)\right) \omega_{0}(g) .}
\end{aligned}
$$

Now we use the relationship between $Q$ and modular functions (10) to convert this expression into

$$
\begin{aligned}
\left(\left|\psi_{B}\left(a_{L}(g)\right)\right|^{1 / 2}\left|\varphi_{B}\left(b_{L}(g)\right)\right|^{-1 / 2}\right. & \\
& \left.\times \int_{A} \nu_{r}^{2}(a)\left|\psi_{B}(a)\right|^{-1} \overline{f_{1}\left(s_{A}\left(a b_{R}(g)\right)\right)} f_{2}\left(a_{R}\left(a b_{R}(g)\right) g^{-1}\right)\right) \omega_{0}(g) .
\end{aligned}
$$

Since $a b_{R}(g)=a a_{L}(g)^{-1} g, a_{R}\left(a b_{R}(g)\right) g^{-1}=b_{L}\left(a a_{L}(g)^{-1} g\right)^{-1} a a_{L}(g)^{-1}$ and $\nu_{r}$ is right-invariant, this expression is equal to the left hand side.

From the formula (18) for $\widehat{\delta}$ and the definitions of $\sigma$ and $\tau$ it easily follows that to prove the third statement it is sufficient to show the equality

$$
\begin{aligned}
\frac{\psi_{A}\left(a_{1} a_{2}\right) \varphi_{A}(b)}{\psi_{A}\left(a_{R}\left(a_{1} a_{2} b\right)\right) \varphi_{B}\left(b_{L}\left(a_{1} a_{2} b\right)\right)} & \\
= & \frac{\psi_{A}\left(a_{1}\right) \varphi_{A}\left(b_{1}\right) Q\left(a_{2} b_{2}\right) \varphi_{B}\left(b_{L}\left(b_{L}^{-1}\left(a_{1} a_{2} b\right) a_{1} b_{1}\right)\right)}{\varphi_{B}\left(b_{L}\left(a_{1} b_{1}\right)\right) \psi_{A}\left(a_{R}\left(a_{1} b_{1}\right)\right) \psi_{A}\left(a_{L}\left(b_{L}^{-1}\left(a_{1} a_{2} b\right) a_{1} b_{1}\right)\right)} \\
& \times \frac{\psi_{A}\left(a_{R}\left(b_{L}^{-1}\left(a_{1} a_{2} b\right) a_{1} b_{1}\right)\right)}{\varphi_{A}\left(b_{R}\left(b_{L}^{-1}\left(a_{1} a_{2} b\right) a_{1} b_{1}\right)\right) Q\left(a_{R}\left(a_{2} b\right) b^{-1} b_{2}\right)},
\end{aligned}
$$

and this is straightforward because of the equalities

$$
\begin{gathered}
b_{L}\left(b_{L}^{-1}\left(a_{1} a_{2} b\right) a_{1} b_{1}\right)=b_{L}^{-1}\left(a_{1} a_{2} b\right) b_{L}\left(a_{1} b_{1}\right), \quad a_{R}\left(b_{L}^{-1}\left(a_{1} a_{2} b\right) a_{1} b_{1}\right)=a_{R}\left(a_{1} b_{1}\right), \\
a_{L}\left(b_{L}^{-1}\left(a_{1} a_{2} b\right) a_{1} b_{1}\right)=a_{R}\left(a_{1} a_{2} b\right) a_{R}^{-1}\left(a_{2} b\right), \quad b_{R}\left(b_{L}^{-1}\left(a_{1} a_{2} b\right) a_{1} b_{1}\right)=b_{L}^{-1}\left(a_{2} b\right) b_{1}, \\
Q\left(a_{2} b_{2}\right)=Q\left(a_{R}\left(a_{2} b\right) b^{-1} b_{2}\right) Q\left(a_{2} b\right),
\end{gathered}
$$

and equation (10). 
The relationships among $h, \widehat{h}$ and $\sigma_{t}$ given in Proposition 6.1 suggest that $\widehat{h}$ can be extended to a GNS mapping and $h$ to a KMS weight on $C_{r}^{*}\left(G_{A}\right)$. This is indeed the case, and was proved in [13] for a general differential groupoid.

Proposition 6.4 ([13]). The mapping $\widehat{h}$ is closable and defines a GNS mapping from $C_{r}^{*}\left(G_{A}\right)$ to $L^{2}(G)$. Consequently, the linear functional $h$ can be extended to a densely defined, lower semicontinuous weight on $C_{r}^{*}\left(G_{A}\right)$, which is a KMS weight with the modular group $\sigma_{t}$.

In the remaining part of this section we show that this weight is rightinvariant. The crucial (although not difficult) step is Lemma 6.5, and then we use results from [14] and [10].

Let $H$ be a Hilbert space. For two vectors $x, y \in H$, we define a linear functional $\eta_{x y}$ on $B(H)$ by $\eta_{x y}(a):=(x \mid a y)$. Then for $a \in B(H \otimes H)$ we define operators (id $\left.\otimes \eta_{x y}\right) a$ and $\left(\eta_{x y} \otimes\right.$ id $) a$ by $\left(z \mid\left(\left(\right.\right.\right.$ id $\left.\left.\left.\otimes \eta_{x y}\right) a\right) t\right):=$ $(z \otimes x \mid a(t \otimes y))$ and $\left(z \mid\left(\left(\eta_{x y} \otimes \mathrm{id}\right) a\right) t\right):=(x \otimes z \mid a(y \otimes t))$.

LEMmA 6.5. Let $\widehat{h}: C_{r}^{*}\left(G_{A}\right) \rightarrow L^{2}(G)$ be the GNS mapping associated with the weight $h$ and $W$ the multiplicative unitary defined in (6). For $x, y \in$ $L^{2}(G)$ and $a \in D(\widehat{h})$ the element $\widehat{h}\left(\left(\mathrm{id} \otimes \eta_{x y}\right) \pi_{\delta}(a)\right)$ belongs to $D(\widehat{h})$ and

$$
\widehat{h}\left(\left(\mathrm{id} \otimes \eta_{x y}\right) \pi_{\delta}(a)\right)=\left(\left(\mathrm{id} \otimes \eta_{x y}\right) W\right) \widehat{h}(a) .
$$

Proof. First, notice that since $\widehat{h}$ is a closed mapping and $\mathcal{A}\left(G_{A}\right)$ is its core, it is enough to prove the formula for $x, y \in \mathcal{D}(G)$ and $a \in \mathcal{A}\left(G_{A}\right)$. Indeed, let $a \in D(\widehat{h})$ and $x=\widehat{h}(a)$. This means that $a=\lim \omega_{n}$ for some $\omega_{n} \in \mathcal{A}\left(G_{A}\right)$ and $x=\lim \widehat{h}\left(\omega_{n}\right)$. It is shown below that $\left(\mathrm{id} \otimes \eta_{x y}\right) \pi_{\delta}\left(\omega_{n}\right)$ is a sequence in $\mathcal{A}\left(G_{A}\right)$ and it converges to (id $\left.\otimes \eta_{x y}\right) \pi_{\delta}(a)$. So

$$
\widehat{h}\left(\left(\mathrm{id} \otimes \eta_{x y}\right) \pi_{\delta}\left(\omega_{n}\right)\right)=\left(\left(\mathrm{id} \otimes \eta_{x y}\right) W\right) \widehat{h}\left(\omega_{n}\right) .
$$

But since $\lim \widehat{h}\left(\omega_{n}\right)=x$, the sequence on the right hand side is convergent to $\left(\left(\mathrm{id} \otimes \eta_{x y}\right) W\right) x$. Therefore $\left(\operatorname{id} \otimes \eta_{x y}\right) \pi_{\delta}(a)$ is in $D(\widehat{h})$ and $\widehat{h}\left(\left(\operatorname{id} \otimes \eta_{x y}\right) \pi_{\delta}(a)\right)=$ $\left(\left(\mathrm{id} \otimes \eta_{x y}\right) W\right) \widehat{h}(a)$. Since $\mathcal{D}(G)$ is dense in $L^{2}(G)$, similar arguments show that it is enough to check the equality for $x, y \in \mathcal{D}(G)$.

Now we compute (id $\left.\otimes \eta_{x y}\right) \pi_{\delta}(\omega)$ for $x, y \in \mathcal{D}(G)$ and $\omega \in \mathcal{A}\left(G_{A}\right)$. Let $\psi_{0}:=\varrho_{0} \otimes \nu_{r}$, where $\varrho_{0}$ is our standard right-invariant half-density defined in (12) and $\nu_{r}$ is as in the definition of $h$. We write $x=: f_{x} \psi_{0}, y=: f_{y} \psi_{0}$ and $\omega=: f \omega_{0}$. With this notation we get $\left(\mathrm{id} \otimes \eta_{x y}\right) \pi_{\delta}(\omega)=f_{x y} \omega_{0}$, where

$$
\begin{aligned}
& f_{x y}(a b) \\
:= & \left|\varphi_{B}(b)\right|^{-1 / 2} \int_{G} \psi_{0}^{2}\left(g_{2}\right) \overline{f_{x}\left(g_{2}\right)}\left|\psi_{B}\left(a_{R}\left(a_{2}^{-1} b\right)\right)\right| f\left(a a_{2} b_{L}\left(a_{2}^{-1} b\right)\right) f_{y}\left(b^{-1} g_{2}\right) .
\end{aligned}
$$


To prove this formula it is enough to show that

$$
\left(z \mid \pi_{\mathrm{id}}\left(f_{x y} \omega_{0}\right) t\right)=\left(z \otimes x \mid \pi_{\delta}(\omega)(t \otimes y)\right) \quad \text { for any } z, t \in \mathcal{D}(G) .
$$

Let $z=: f_{z} \psi_{0}$ and $t=: f_{t} \psi_{0}$. From the formula (18) for $\widehat{\delta}$ we get

$$
\begin{aligned}
& \left(z \otimes x \mid \pi_{\delta}(\omega)(t \otimes y)\right)=\int_{G \times G}\left(\psi_{0} \otimes \psi_{0}\right)^{2}\left(g_{1}, g_{2}\right) \overline{f_{z}\left(g_{1}\right) f_{x}\left(g_{2}\right)} \\
& \quad \times \int_{B} \mu_{L}^{2}(b)\left|\varphi_{B}\left(b_{L}\left(a_{2} b\right)\right)\right|^{-1 / 2} f\left(a_{1} a_{2} b\right) f_{t}\left(b_{L}\left(a_{1} a_{2} b\right)^{-1} a_{1} b_{1}\right) f_{y}\left(a_{R}\left(a_{2} b\right) b^{-1} b_{2}\right) \\
& =\int_{G} \psi_{0}^{2}\left(g_{1}\right) \overline{f_{z}\left(g_{1}\right)} \int_{G} \psi_{0}^{2}\left(g_{2}\right) \overline{f_{x}\left(g_{2}\right)} \\
& \quad \times \int_{B} \mu_{L}^{2}(b)\left|\varphi_{B}\left(b_{L}\left(a_{2} b\right)\right)\right|^{-1 / 2} f\left(a_{1} a_{2} b\right) f_{t}\left(b_{L}\left(a_{1} a_{2} b\right)^{-1} a_{1} b_{1}\right) f_{y}\left(a_{R}\left(a_{2} b\right) b^{-1} b_{2}\right),
\end{aligned}
$$

where $g_{1}=a_{1} b_{1}, g_{2}=a_{2} b_{2}$. Applying the diffeomorphism $B \ni b \mapsto b_{L}\left(a_{2} b\right)$ $\in B$, we can rewrite the integral over $B$ as

$$
\begin{aligned}
\int_{B} \mu_{L}^{2}(b)\left|\varphi_{B}(b)\right|^{-1 / 2} \mid & \psi_{B}\left(a_{R}\left(a_{1}^{-1} b\right)\right) \mid \\
& \times f\left(a_{1} a_{2} b_{L}\left(a_{2}^{-1} b\right)\right) f_{y}\left(b^{-1} a_{2} b_{2}\right) f_{t}\left(b_{L}\left(a_{1} b\right)^{-1} a_{1} b_{1}\right),
\end{aligned}
$$

and interchanging the order of integration we get

$$
\begin{aligned}
& \left(z \otimes x \mid \pi_{\delta}(\omega)(t \otimes y)\right)=\int_{G} \psi_{0}^{2}\left(g_{1}\right) \overline{f_{z}\left(g_{1}\right)} \int_{B} \mu_{L}^{2}(b)\left|\varphi_{B}(b)\right|^{-1 / 2} \\
& \times\left[\int_{G} \psi_{0}^{2}\left(g_{2}\right) \overline{f_{x}\left(g_{2}\right)}\left|\psi_{B}\left(a_{R}\left(a_{1}^{-1} b\right)\right)\right| f\left(a_{1} a_{2} b_{L}\left(a_{2}^{-1} b\right)\right) f_{y}\left(b^{-1} g_{2}\right)\right] f_{t}\left(b_{L}\left(a_{1} b\right)^{-1} g_{1}\right) .
\end{aligned}
$$

Comparing this with the formula (13) for multiplication in $G_{A}$ and the definition of $f_{x y}$ we obtain the result.

To prove the lemma it remains to show that

$$
\left(z \mid \widehat{h}\left(f_{x y} \omega_{0}\right)\right)=(z \otimes x \mid W(\widehat{h}(\omega) \otimes y)) .
$$

Using the definition of $\widehat{h}$ we get $\widehat{h}\left(f \omega_{0}\right)=\widehat{f} \psi_{0}$ for $\widehat{f}(g):=f(g)\left|\psi_{B}\left(a_{R}(g)\right)\right|^{-1 / 2}$. Let us compute the right hand side of our equality:

$$
\begin{aligned}
(z \otimes x \mid W(\widehat{h}(\omega) \otimes y)) & =\int_{G \times G}\left(\psi_{0} \otimes \psi_{0}\right)^{2}\left(g_{1}, g_{2}\right) \overline{f_{z}\left(g_{1}\right) f_{x}\left(g_{2}\right)}\left[W\left(\widehat{f} \otimes f_{y}\right)\right]\left(g_{1}, g_{2}\right) \\
& =\int_{G} \psi_{0}^{2}\left(g_{1}\right) \overline{f_{z}\left(g_{1}\right)} \int_{G} \psi_{0}^{2}\left(g_{2}\right) \overline{f_{x}\left(g_{2}\right)}\left[W\left(\widehat{f} \otimes f_{y}\right)\right]\left(g_{1}, g_{2}\right) .
\end{aligned}
$$

Thus we have to prove the equality

$$
(* *) \quad f_{x y}(g)\left|\psi_{B}\left(a_{R}(g)\right)\right|^{-1 / 2}=\int_{G} \psi_{0}^{2}\left(g_{2}\right) \overline{f_{x}\left(g_{2}\right)}\left[W\left(\widehat{f} \otimes f_{y}\right)\right]\left(g, g_{2}\right),
$$

where $W\left(f \psi_{0} \otimes k \psi_{0}\right)=: W(f \otimes k)\left(\psi_{0} \otimes \psi_{0}\right)$. 
The formula for the action of $W$ was given after Lemma 4.4. Notice that now $\psi_{0}$ is not the same as $\psi_{0}$ defined before that lemma, so the expressions for $W\left(\widehat{f} \otimes f_{y}\right)$ are different. But since both $\psi_{0}$ 's can be easily compared, we immediately deduce the needed formula from the one in Lemma 4.4:

$$
\begin{aligned}
{[W(\widehat{f}} & \left.\left.\otimes f_{y}\right)\right]\left(g, g_{2}\right) \\
& =\left|\varphi_{B}\left(b_{R}(g)\right) \psi_{B}\left(a_{L}\left(b_{R}(g)^{-1} g_{2}\right)\right)\right|^{-1 / 2} \widehat{f}\left(g a_{L}\left(b_{R}(g)^{-1} g_{2}\right)\right) f_{y}\left(b_{R}(g)^{-1} g_{2}\right) .
\end{aligned}
$$

To verify the equality $(* *)$ use this expression and the expressions for $f_{x y}$ (see $(32))$ and $\widehat{f}$. This completes the proof of the lemma.

Now we can prove right-invariance of $h$ with respect to positive vector functionals in a GNS space for $h$. It follows immediately from

Proposition 6.6. For any $a \in D(\widehat{h})$ and $x \in L^{2}(G)$,

$$
h\left(\left(\mathrm{id} \otimes \eta_{x x}\right) \pi_{\delta}\left(a^{*} a\right)\right)=\|x\|^{2} h\left(a^{*} a\right) .
$$

Proof. Let $e_{i}$ be an orthonormal basis in $L^{2}(G)$. For $x \in L^{2}(G)$ let

$$
B_{n}:=\sum_{i=1}^{n}\left[\left(\operatorname{id} \otimes \eta_{e_{i} x}\right) \pi_{\delta}(a)\right]^{*}\left[\left(\operatorname{id} \otimes \eta_{e_{i} x}\right) \pi_{\delta}(a)\right] .
$$

Then $B_{n} \in C_{r}^{*}\left(G_{A}\right)$ and $B_{n}$ converges strictly to (id $\left.\otimes \eta_{x x}\right) \pi_{\delta}\left(a^{*} a\right)$ (see Lemma 9.5 of [14]). On the other hand,

$$
\begin{aligned}
h\left(B_{n}\right) & =\sum_{i=1}^{n}\left(\widehat{h}\left[\left(\mathrm{id} \otimes \eta_{e_{i} x}\right) \pi_{\delta}(a)\right] \mid \widehat{h}\left[\left(\mathrm{id} \otimes \eta_{e_{i} x}\right) \pi_{\delta}(a)\right]\right) \\
& =\sum_{i=1}^{n}\left(\left[\left(\mathrm{id} \otimes \eta_{e_{i} x}\right) W\right] \widehat{h}(a) \mid\left[\left(\mathrm{id} \otimes \eta_{e_{i} x}\right) W\right] \widehat{h}(a)\right) \\
& =\left(\widehat{h}(a) \mid \sum_{i=1}^{n}\left[\left(\mathrm{id} \otimes \eta_{e_{i} x}\right) W\right]^{*}\left[\left(\mathrm{id} \otimes \eta_{e_{i} x}\right) W\right] \widehat{h}(a)\right) .
\end{aligned}
$$

Because $W \in M\left(C B\left(L^{2}(G)\right) \otimes C_{r}^{*}\left(G_{A}\right)\right)$ (see remarks after Lemma 3.3), it follows that the sequence $\sum_{i=1}^{n}\left[\left(\mathrm{id} \otimes \eta_{e_{i} x}\right) W\right]^{*}\left[\left(\mathrm{id} \otimes \eta_{e_{i} x}\right) W\right]$ converges strongly to $\left(\right.$ id $\left.\otimes \eta_{x x}\right)\left(W^{*} W\right)=\|x\|^{2} I$. Therefore $h\left(B_{n}\right)$ converges to $\|x\|^{2} h\left(a^{*} a\right)$. Since the weight $h$ is strictly lower semicontinuous, we get the result.

The last step is to prove that this is enough for full right-invariance. To this end we use the following

TheOREM 6.7 ([10]). Let $h$ be a densely defined, lower semicontinuous weight on a separable $C^{*}$-algebra $A$ and $\left(H, \eta_{h}, \pi_{h}\right)$ be a GNS triple. There exists a sequence of vectors $\Omega_{n} \in H$ such that:

1. $\pi_{h}(A) \Omega_{n}$ and $\pi_{h}(A) \Omega_{m}$ are orthogonal for $m \neq n$.

2. $a \in D\left(\eta_{h}\right) \Leftrightarrow \sum\left\|\pi_{h}(a) \Omega_{n}\right\|^{2}<\infty$.

3. $\eta_{h}(a)=\sum \pi_{h}(a) \Omega_{n}$.

4. $H=\bigoplus H_{n}, H_{n}:=\overline{\pi_{h}(A) \Omega_{n}}$. 
To simplify notation we put $\eta_{i}:=\eta_{\Omega_{i} \Omega_{i}}$ and, for $a, b \in D(\widehat{h}), \eta_{i a}:=$ $\eta_{\Omega_{i} \widehat{h}(a)}, \eta_{a b}:=\eta_{\widehat{h}(a) \widehat{h}(b)}$. From Lemma 6.5 and the theorem above we easily obtain three useful formulae:

$$
\begin{aligned}
\left(\eta_{i} \otimes \mathrm{id}\right) \Delta(a) & =\left(\eta_{i a} \otimes \mathrm{id}\right) W, \quad a \in D(\widehat{h}), \\
\Delta(a)\left(\Omega_{n} \otimes x\right) & =\left(p_{n} \otimes I\right) W(\widehat{h}(a) \otimes x), \quad a \in D(\widehat{h}), \\
\left(\eta_{i\left(a^{*} a\right)} \otimes \mathrm{id}\right) W & =\left(\eta_{a} \otimes \mathrm{id}\right)\left(W^{*}\left(p_{i} \otimes I\right) W\right),
\end{aligned}
$$

where $p_{i}$ denotes projection onto the closure of $C_{r}^{*}\left(G_{A}\right) \Omega_{i}$. Indeed, let $x, y, z$ $\in L^{2}(G)$. Then

$$
\begin{aligned}
& \left(x \mid\left(\eta_{i a} \otimes \mathrm{id}\right) W y\right)=\left(\Omega_{i} \otimes x \mid W(\widehat{h}(a) \otimes y)\right)=\left(\Omega_{i} \mid\left(\mathrm{id} \otimes \eta_{x y}\right) W \widehat{h}(a)\right) \\
& =\left(\Omega_{i} \mid \widehat{h}\left(\left(\mathrm{id} \otimes \eta_{x y}\right) \Delta(a)\right)\right)=\left(\Omega_{i} \mid\left(\mathrm{id} \otimes \eta_{x y}\right) \Delta(a) \Omega_{i}\right)=\left(x \mid\left(\eta_{i} \otimes \mathrm{id}\right) \Delta(a) y\right),
\end{aligned}
$$

and this proves the first formula. To prove the second, compute

$$
\begin{aligned}
(x \otimes z \mid W(\widehat{h}(a) \otimes y)) & =\left(x \mid\left(\mathrm{id} \otimes \eta_{z y}\right) W \widehat{h}(a)\right)=\left(x \mid \widehat{h}\left(\left(\mathrm{id} \otimes \eta_{z y}\right) \Delta(a)\right)\right) \\
& =\sum_{n}\left(x \mid\left(\operatorname{id} \otimes \eta_{z y}\right) \Delta(a) \Omega_{n}\right),
\end{aligned}
$$

so

$$
\begin{aligned}
\left(x \otimes z \mid\left(p_{n} \otimes I\right) W(\widehat{h}(a) \otimes y)\right) & =\left(x \mid\left(\mathrm{id} \otimes \eta_{z y}\right) \Delta(a) \Omega_{n}\right) \\
& =\left(x \otimes z \mid \Delta(a)\left(\Omega_{n} \otimes y\right)\right) .
\end{aligned}
$$

And the third formula

$$
\begin{aligned}
& \left(z \mid\left(\eta_{i\left(a^{*} a\right)} \otimes \mathrm{id}\right) W y\right)=\left(\Omega_{i} \otimes z \mid W\left(\widehat{h}\left(a^{*} a\right) \otimes y\right)\right)=\left(\Omega_{i} \otimes z \mid W\left(a^{*} \otimes I\right)(\widehat{h}(a) \otimes y)\right) \\
& \quad=\left(\Omega_{i} \otimes z \mid \Delta\left(a^{*}\right) W(\widehat{h}(a) \otimes t)\right)=\left(\Delta(a)\left(\Omega_{i} \otimes z\right) \mid W(\widehat{h}(a) \otimes y)\right) \\
& \quad=\left(\widehat{h}(a) \otimes z \mid W^{*}\left(p_{i} \otimes I\right) W \widehat{h}(a) \otimes y\right)=\left(z \mid\left(\left(\eta_{a} \otimes \mathrm{id}\right) W^{*}\left(p_{i} \otimes I\right) W\right) y\right) .
\end{aligned}
$$

Now, let $\varphi$ be a positive linear functional on $C_{r}^{*}\left(G_{A}\right)$ and $\left(K, \pi_{\varphi}, \Omega_{\varphi}\right)$ the associated GNS triple. We compute

$$
\begin{aligned}
\eta_{i}\left((\mathrm{id} \otimes \varphi) \Delta\left(a^{*} a\right)\right) & =\varphi\left(\left(\eta_{i} \otimes \mathrm{id}\right) \Delta\left(a^{*} a\right)\right)=\varphi\left(\left(\eta_{i\left(a^{*} a\right)} \otimes \mathrm{id}\right) W\right) \\
& =\varphi\left(\left(\eta_{a} \otimes \mathrm{id}\right)\left(W^{*}\left(p_{i} \otimes I\right) W\right)\right) .
\end{aligned}
$$

But since $W \in M\left(C B\left(L^{2}(G)\right) \otimes C_{r}^{*}\left(G_{A}\right)\right)$ and $\sum_{i=1}^{n} p_{i}$ converges strictly (in $B(H)=M(C B(H)))$ to $I$, we conclude that $\sum_{i} \eta_{i}\left((\operatorname{id} \otimes \varphi) \Delta\left(a^{*} a\right)\right)=$ $h\left(a^{*} a\right) \varphi(I)$. This shows that $h\left((\operatorname{id} \otimes \varphi) \Delta\left(a^{*} a\right)\right)$ is finite and equal to $h\left(a^{*} a\right) \varphi(I)$. This way we have proved:

Proposition 6.8. Let $a \in D(\widehat{h})$ and $\varphi$ be a positive linear functional on $C_{r}^{*}\left(G_{A}\right)$. Then

$$
h\left((\operatorname{id} \otimes \varphi) \Delta\left(a^{*} a\right)\right)=h\left(a^{*} a\right) \varphi(I) .
$$

Therefore, the weight $h$ is right-invariant. 
7. Putting all together. In this section we lift some objects from Section 5 to the $C^{*}$-algebraic level and review the structure we got. Let us start by showing that $R$ defines a *-antiautomorphism of $C_{r}^{*}\left(G_{A}\right)$. To this end it is enough to prove its continuity. This will follow immediately from

Lemma 7.1. There exists an antiunitary operator $\widehat{J}$ on $L^{2}\left(G_{A}\right)$ such that

$$
\pi_{\text {id }}(R \omega)=\widehat{J} \pi_{\text {id }}\left(\omega^{*}\right) \widehat{J}, \quad \omega \in \mathcal{A}\left(G_{A}\right) .
$$

Proof. Since $s_{B}$ (inverse of the groupoid $G_{B}$ ) is a diffeomorphism of $G_{A}$, it defines a unitary operator on $L^{2}\left(G_{A}\right)$ (by push-forward of half-densities), which we also denote by $s_{B}$. On $L^{2}\left(G_{A}\right)$ there is a canonical antiunitary involution, namely complex conjugation: ${ }^{-}$. We define $\widehat{J}:={ }^{-} \circ s_{B}$ and check that this is the right choice. We choose a non-vanishing, real half-density $\psi_{0}=\varrho_{0} \otimes \nu$ on $G_{A}$ as before Lemma 4.4. Then a short computation shows that

$$
\begin{array}{r}
s_{B}(Y g \wedge g X)=\psi_{B}\left(a_{L}(g)\right)^{-1} \psi_{A}\left(a_{R}(g)\right)(-1)^{\operatorname{dim} A}\left(Y s_{B}(g) \wedge s_{B}(g) X\right) \\
\text { for } X \in \Lambda^{\max } T_{e} A, Y \in \Lambda^{\max } T_{e} B .
\end{array}
$$

From this formula we deduce that

$$
\left(s_{B} \psi_{0}\right)(g)=\left|\psi_{B}\left(a_{L}(g)\right)\right|^{1 / 2}\left|\psi_{A}\left(a_{R}(g)\right)\right|^{1 / 2} \psi_{0}(g) .
$$

Defining $s_{B} \psi$ for any smooth, compactly supported function $\psi$ on $G_{A}$ by $s_{B}\left(\psi \psi_{0}\right)=:\left(s_{B} \psi\right) \psi_{0}$ we get

$$
\left(s_{B} \psi\right)(g)=\left|\psi_{B}\left(a_{L}(g)\right)\right|^{1 / 2}\left|\psi_{A}\left(a_{R}(g)\right)\right|^{1 / 2} \psi\left(s_{B}(g)\right) .
$$

As usual, writing $\omega=f \omega_{0}$, we have to show $(R f) * \psi=\overline{s_{B}\left(f^{*} * \overline{s_{B} \psi}\right)}$. We compute the left hand side using the formulae (24) for $R$ and the multiplication (13):

$$
\begin{aligned}
& ((R f) * \psi)(g)=\int_{B} \mu_{L}^{2}(b)(R f)\left(a_{L}(g) b\right) \psi\left(b_{L}\left(a_{L}(g) b\right)^{-1} g\right) \\
& =\left|\psi_{B}\left(a_{L}(g)\right)\right|^{1 / 2} \int_{B} \mu_{L}^{2}(b)\left|\psi_{B}\left(a_{R}\left(a_{L}(g) b\right)\right)\right|^{1 / 2} f\left(b^{-1} a_{L}(g)^{-1}\right) \psi\left(b_{L}\left(a_{L}(g) b\right)^{-1} g\right) .
\end{aligned}
$$

And the right hand side equals

$$
\begin{aligned}
\overline{s_{B}\left(f^{*} * \overline{s_{B} \psi}\right)}(g)= & \left|\psi_{B}\left(a_{L}(g)\right)\right|^{1 / 2}\left|\psi_{A}\left(a_{R}(g)\right)\right|^{1 / 2} \overline{\left(f^{*} * \overline{s_{B} \psi}\right)\left(s_{B}(g)\right)} \\
= & \left|\psi_{B}\left(a_{L}(g)\right)\right|^{1 / 2}\left|\psi_{A}\left(a_{R}(g)\right)\right|^{1 / 2} \\
& \times \int_{B} \mu_{L}^{2}(b) \overline{f^{*}}\left(a_{L}\left(s_{B}(g)\right) b\right)\left(s_{B} \psi\right)\left(b_{L}\left(a_{L}\left(s_{B}(g)\right) b\right)^{-1} s_{B}(g)\right) \\
= & \left|\psi_{B}\left(a_{L}(g)\right)\right|^{1 / 2}\left|\psi_{A}\left(a_{R}(g)\right)\right|^{1 / 2} \\
& \times \int_{B} \mu_{L}^{2}(b) f\left(s_{A}\left(a_{L}(g)^{-1} b\right)\right)\left(s_{B} \psi\right)\left(b_{L}\left(a_{L}(g)^{-1} b\right)^{-1} s_{B}(g)\right)
\end{aligned}
$$




$$
\begin{aligned}
= & \left|\psi_{B}\left(a_{L}(g)\right)\right|^{1 / 2}\left|\psi_{A}\left(a_{R}(g)\right)\right|^{1 / 2} \\
& \times \int_{B} \mu_{L}^{2}(b) f\left(b_{L}\left(a_{L}(g)^{-1} b\right)^{-1} a_{L}(g)^{-1}\right)\left(s_{B} \psi\right)\left(b_{L}\left(a_{L}(g)^{-1} b\right)^{-1} s_{B}(g)\right) .
\end{aligned}
$$

Now apply the diffeomorphism $B \ni b \mapsto b_{L}\left(a_{L}(g)^{-1} b\right) \in B$ to the integral over $B$ to convert it into

$$
\begin{aligned}
\left|\psi_{B}\left(a_{L}(g)\right)\right|^{1 / 2}\left|\psi_{A}\left(a_{R}(g)\right)\right|^{1 / 2} & \\
& \times \int_{B} \mu_{L}^{2}(b)\left|\psi_{B}\left(a_{R}\left(a_{L}(g) b\right)\right)\right| f\left(b^{-1} a_{L}(g)^{-1}\right)\left(s_{B} \psi\right)\left(b^{-1} s_{B}(g)\right)
\end{aligned}
$$

and use the definition of $s_{B} \psi$ to conclude that this is equal to the left hand side.

Now we pass to the group $\tau_{t}$. The following proposition was proved in [13]:

Proposition 7.2. Let $\Gamma$ be a differential groupoid and $\sigma: \Gamma \rightarrow] 0, \infty[a$ smooth cocycle. The mapping $\mathcal{A}(\Gamma) \ni \omega \mapsto \sigma_{t}(\omega):=\sigma^{i t} \omega \in \mathcal{A}(\Gamma)$ extends to a strongly continuous one-parameter group on $C_{r}^{*}(\Gamma)$. Let $\sigma_{i / 2}$ be its analytic generator. Then $\mathcal{A}(\Gamma)$ is a core for $\sigma_{i / 2}$ and $\sigma_{i / 2}(\omega)=\sigma^{-1 / 2} \omega$ for $\omega \in$ $\mathcal{A}(\Gamma)$.

From this proposition we infer that $\tau_{t}$ defined by (23) extends to a strongly continuous one-parameter group on $C_{r}^{*}\left(G_{A}\right)$. Moreover, from Proposition 5.1 this group commutes with $R$. Since now we know that $R \tau_{t}$ and $\sigma_{t}$ are continuous, using Lemma 5.4 and Proposition 6.3, we easily obtain the following equalities on $C_{r}^{*}\left(G_{A}\right)$ :

$$
\Delta \tau_{t}=\left(\tau_{t} \otimes \tau_{t}\right) \Delta, \quad \Delta R=\sim(R \otimes R) \Delta, \quad \Delta \sigma_{t}=\left(\sigma_{t} \otimes \tau_{t}\right) \Delta
$$

We finish this work with a short résumé. Let $(G ; A, B)$ be a double Lie group.

- There are naturally defined differential groupoids $G_{A}, G_{B}$ over $A$ and $B$ respectively. Let $C:=C_{r}^{*}\left(G_{A}\right)$ be the reduced $C^{*}$-algebra of $G_{A}$.

- The relation $\delta:=m_{B}^{T}$ defines $\Delta \in \operatorname{Mor}(C, C \otimes C)$ which is a comultiplication in the sense of the theory of quantum groups.

- The inverse of the group $G$ defines an involutive *-antiautomorphism $R$ of $C$.

- There is a strongly continuous one-parameter group of $*$-automorphisms $\tau_{t}$ on $C$, which commutes with $R$.

- There exists a right-invariant, densely defined, lower semicontionuous weight $h$ on $C$ which is a KMS weight with a modular group $\sigma_{t}$. Moreover the weight $h$ is $\tau$-invariant.

- The groups $\sigma_{t}$ and $\tau_{t}$ commute.

- $\Delta \tau_{t}=\left(\tau_{t} \otimes \tau_{t}\right) \Delta, \quad \Delta R=\sim(R \otimes R) \Delta, \quad \Delta \sigma_{t}=\left(\sigma_{t} \otimes \tau_{t}\right) \Delta$. 
Because the positions of the groups $A$ and $B$ in a DLG $(G ; A, B)$ are completely symmetric, in fact, there are two quantum groups based on $C_{r}^{*}\left(G_{A}\right)$ and $C_{r}^{*}\left(G_{B}\right)$ respectively. One can think of them as duals, but the precise sense of this duality is to be understood. If these algebras coincide with the algebras defined by the multiplicative operator $W$ in the "standard way" (see Appendix B), then one can say that this is the meaning of duality. However, here we have more, namely, there is a natural duality (in fact two of them) between $\mathcal{A}\left(G_{A}\right)$ and $\mathcal{A}\left(G_{B}\right)$ (see Appendix A). This duality enables us to think about these algebras as "dual Hopf algebras". There is also a class of representations of (say) $C_{r}^{*}\left(G_{B}\right)$, the ones coming from morphisms of differential groupoids $G_{B} \rightarrow \Gamma$, which define, via $W$, representations of the quantum group based on $C_{r}^{*}\left(G_{A}\right)$.

\section{Appendices}

A. Geometric interpretation of the function $Q$. Let $V$ be a finitedimensional vector space (over $\mathbb{C}$ or $\mathbb{R}$ ). Suppose we are given four subspaces $L_{1}, L_{2}, R_{1}, R_{2} \subset V$ such that $\operatorname{dim} L_{1}=\operatorname{dim} R_{1}, \operatorname{dim} L_{2}=\operatorname{dim} R_{2}$ and $V=$ $L_{1} \oplus R_{2}=R_{1} \oplus L_{2}=L_{1} \oplus L_{2}=R_{1} \oplus R_{2}$. Moreover let $\lambda_{i}, \varrho_{i}, i=1,2$, be half-densities on $L_{i}$ and $R_{i}$ respectively. The quadruple $\left(\lambda_{1}, \varrho_{1}, \lambda_{2}, \varrho_{2}\right)$ defines two densities $d_{1}, d_{2}$ on $V$ as follows: $d_{1}:=\left(\lambda_{1} \otimes \varrho_{2}\right)\left(\lambda_{2} \otimes \varrho_{1}\right)$ and $d_{2}:=\left(\lambda_{1} \otimes \lambda_{2}\right)\left(\varrho_{1} \otimes \varrho_{2}\right)$. One can check that $d_{1}, d_{2}$ depend only on $\lambda_{1} \otimes \varrho_{1}$ and $\lambda_{2} \otimes \varrho_{2}$ and the dependence is bilinear. Therefore, $d_{1}, d_{2}$ are actually bilinear mappings $d_{1}, d_{2}:\left(\Omega^{1 / 2}\left(L_{1}\right) \otimes \Omega^{1 / 2}\left(R_{1}\right)\right) \times\left(\Omega^{1 / 2}\left(L_{2}\right) \otimes \Omega^{1 / 2}\left(R_{2}\right)\right) \rightarrow$ $\Omega^{1}(V)$. Since the space $\Omega^{1}(V)$ is one-dimensional we infer that $d_{1}=c d_{2}$ for some $c \in \mathbb{C}$. To find the constant $c$ it is sufficient to compare $d_{1}$ and $d_{2}$ on some basis in $V$. Let us recall that a p-density $d$ on $V$ is a mapping $d: V^{n}:=V \times \cdots \times V \rightarrow \mathbb{C}, n=\operatorname{dim} V$, which satisfies the condition

$$
d\left(v_{i_{1}} A_{i_{1} 1}, \ldots, v_{i_{n}} A_{i_{n} n}\right)=|\operatorname{det} A|^{p} d\left(v_{1}, \ldots, v_{n}\right) \quad \text { (summation), }
$$

for any $A \in M_{n \times n}(\mathbb{R})$ (or $A \in M_{n \times n}(\mathbb{C})$ ) and any $v_{1}, \ldots, v_{n} \in V$. If $\left(v_{1}, \ldots, v_{n}\right)=: \mathbf{v}$ is a basis in $V$ then we write the above condition as $d(\mathbf{v} A)=|\operatorname{det} A|^{p} d(\mathbf{v})$.

Choose bases $\mathbf{l}:=\left(l_{1}, \ldots, l_{n}\right), \mathbf{r}:=\left(r_{1}, \ldots, r_{n}\right), \widetilde{\mathbf{l}}:=\left(\widetilde{l}_{1}, \ldots, \widetilde{l}_{m}\right), \widetilde{\mathbf{r}}:=$ $\left(\widetilde{r}_{1}, \ldots, \widetilde{r}_{m}\right)$ in $L_{1}, R_{1}, L_{2}, R_{2}$, respectively. Let $I$ denote the identity matrix (of appropriate dimension) and let matrices $A, B, C, D, J, K, G, H, M$, $M_{1}, M_{2}$ be defined by

$$
\begin{gathered}
(\mathbf{l}, \widetilde{\mathbf{l}})=:(\mathbf{r}, \widetilde{\mathbf{r}})\left(\begin{array}{cc}
A & C \\
B & D
\end{array}\right)=:(\mathbf{r}, \widetilde{\mathbf{r}}) M \\
(\mathbf{l}, \widetilde{\mathbf{l}})=:(\mathbf{r}, \widetilde{\mathbf{l}})\left(\begin{array}{cc}
J & 0 \\
K & I
\end{array}\right)=(\mathbf{r}, \widetilde{\mathbf{l}}) M_{1}, \quad(\mathbf{l}, \widetilde{\mathbf{l}})=:(\mathbf{l}, \widetilde{\mathbf{r}})\left(\begin{array}{cc}
I & G \\
0 & H
\end{array}\right)=(\mathbf{l}, \widetilde{\mathbf{r}}) M_{2} .
\end{gathered}
$$


Now we can compare $d_{1}$ and $d_{2}$ :

$$
\begin{aligned}
d_{1}(\mathbf{l}, \widetilde{\mathbf{l}}) & :=\left(\lambda_{1} \otimes \varrho_{2}\right)(\mathbf{l}, \widetilde{\mathbf{l}})\left(\lambda_{2} \otimes \varrho_{1}\right)(\mathbf{l}, \widetilde{\mathbf{l}})=\left(\lambda_{1} \otimes \varrho_{2}\right)\left((\mathbf{l}, \widetilde{\mathbf{r}}) M_{2}\right)\left(\lambda_{2} \otimes \varrho_{1}\right)\left((\mathbf{r}, \widetilde{\mathbf{l}}) M_{1}\right) \\
& =|\operatorname{det} H|^{1 / 2}|\operatorname{det} J|^{1 / 2} \lambda_{1}(\mathbf{l}) \varrho_{2}(\widetilde{\mathbf{r}}) \lambda_{2}(\widetilde{\mathbf{l}}) \varrho_{1}(\mathbf{r}), \\
d_{2}(\mathbf{l}, \widetilde{\mathbf{l}}): & =\left(\lambda_{1} \otimes \lambda_{2}\right)(\mathbf{l}, \widetilde{\mathbf{l}})\left(\varrho_{1} \otimes \varrho_{2}\right)(\mathbf{l}, \widetilde{\mathbf{l}})=\lambda_{1}(\mathbf{l}) \lambda_{2}(\widetilde{\mathbf{l}})\left(\varrho_{1} \otimes \varrho_{2}\right)((\mathbf{r}, \widetilde{\mathbf{r}}) M) \\
& =|\operatorname{det} M|^{1 / 2} \lambda_{1}(\mathbf{l}) \lambda_{2}(\widetilde{\mathbf{l}}) \varrho_{1}(\mathbf{r}) \varrho_{2}(\widetilde{\mathbf{r}}) .
\end{aligned}
$$

Hence

$$
d_{2}=\frac{|\operatorname{det} M|^{1 / 2}}{|\operatorname{det} H|^{1 / 2}|\operatorname{det} J|^{1 / 2}} d_{1}
$$

But from the equalities

$$
\mathbf{l}=\mathbf{r} A+\widetilde{\mathbf{r}} B, \quad \widetilde{\mathbf{l}}=\mathbf{r} C+\widetilde{\mathbf{r}} D, \quad \mathbf{l}=\mathbf{r} J+\widetilde{\mathbf{l}} K, \quad \widetilde{\mathbf{l}}=\mathbf{l} G+\widetilde{\mathbf{r}} H,
$$

we infer that $C=A G, B=D K, J=A(I-G K), D=(I-K G)$ and we can write

$$
d_{2}=\left|\frac{\operatorname{det} A \operatorname{det} D}{\operatorname{det} M}\right|^{1 / 2} d_{1} .
$$

Now for a DLG $(G ; A, B)$, fix a point $g \in G$ and define

$$
L_{1}:=T_{g}^{l} G_{B}, \quad L_{2}=T_{g}^{l} G_{A}, \quad R_{1}:=T_{g}^{r} G_{B}, \quad R_{2}:=T_{g}^{r} G_{A}, \quad V:=T_{g} G .
$$

Let $\mathbf{X}:=\left(X_{1}, \ldots, X_{n}\right), \mathbf{Y}=\left(Y_{1}, \ldots, Y_{m}\right)$ be bases in $T_{e} A$ and $T_{e} B$, respectively. Then $g \mathbf{X}, g \mathbf{Y}, \mathbf{X} g, \mathbf{Y} g$ are bases in $L_{1}, L_{2}, R_{1}, R_{2}$, respectively. Moreover, if $M=\left(\begin{array}{ll}A & C \\ B & D\end{array}\right)$ is the matrix of the adjoint representation of $g$ in $(\mathbf{X}, \mathbf{Y})$, then $(g \mathbf{X}, g \mathbf{Y})=(\mathbf{X} g, \mathbf{Y} g) M$. Comparing this with the definition of $Q$ we conclude that $d_{1}=|Q(g)|^{1 / 2} d_{2}$.

In this way we see that the function $Q$ relates two natural dualities between $\mathcal{A}\left(G_{A}\right)$ and $\mathcal{A}\left(G_{B}\right)$ given, for $\omega_{A}=\lambda_{A} \otimes \varrho_{A} \in \mathcal{A}\left(G_{A}\right)$ and $\omega_{B}=$ $\lambda_{B} \otimes \varrho_{B} \in \mathcal{A}\left(G_{B}\right)$, by

$$
\left\langle\omega_{A}, \omega_{B}\right\rangle_{1}:=\int_{G}\left(\lambda_{A} \otimes \varrho_{B}\right)\left(\lambda_{B} \otimes \varrho_{A}\right), \quad\left\langle\omega_{A}, \omega_{B}\right\rangle_{2}:=\int_{G}\left(\lambda_{A} \otimes \lambda_{B}\right)\left(\varrho_{A} \otimes \varrho_{B}\right) .
$$

B. Comparison with the standard approach. Let $W \in B(H \otimes H)$ be a multiplicative unitary operator. As shown by Baaj and Skandalis [1] the set

$$
C:=\overline{\left\{(\eta \otimes \mathrm{id}) W: \eta \in B(H)_{*}\right\}}=\overline{\operatorname{span}\left\{\left(\eta_{x y} \otimes \mathrm{id}\right) W: x, y \in H\right\}}
$$

(bar denotes norm closure) is a $C^{*}$-algebra with a comultiplication. This is the "standard procedure".

In this appendix we consider the following problem. For a DLG $(G ; A, B)$ there is a multiplicative unitary (manageable) $W \in B\left(L^{2}(G) \otimes L^{2}(G)\right)$ and we have the $C^{*}$-algebra $C$ defined as above. It is easy to see that in the definition of $C$, it is enough to consider vectors $x, y$ from a dense subspace of $H$. So a "typical" element of $C$ is obtained from two vectors $x, y$ by $a_{x y}:=\left(\eta_{x y} \otimes \mathrm{id}\right) W$, in particular it is defined by two smooth half-densities 
on $G$ with compact support. On the other hand, we have the groupoid $G_{A}$ and its reduced $C^{*}$-algebra defined in a different way, and its "typical" elements are bidensities on $G_{A}$. We can suppose that those algebras are in fact the same. We will not prove this equality here, but we will show that $C \subset C_{r}^{*}\left(G_{A}\right)$. From the remark above, it follows that we have to interpret elements $\left(\eta_{x y} \otimes \mathrm{id}\right) W$ as elements of $\mathcal{A}\left(G_{A}\right)$ for $x, y$ smooth with compact support. The question is: how from two smooth half-densities on $G$ and $W$ can we get an element of $\mathcal{A}\left(G_{A}\right)$ ?

Suppose we are given two half-densities $\varphi, \psi$ with compact support on $G$. Let $U$ be the bisection implementing $W$ and let $g \in G$ be given. Consider the set $U(g, A) \in G \times G$. It is easy to see that $U(g, A)=\delta(g)$ (recall that $\left.\delta=m_{B}^{T}\right)$. Let $\left(g_{1}, g_{2}\right) \in \delta(g)$. There are the following natural isomorphisms:

$$
\begin{aligned}
\Omega^{1 / 2}\left(T_{g_{1}} G\right) & \simeq \Omega^{1 / 2}\left(T_{b_{L}(g)} B\right) \otimes \Omega^{1 / 2}\left(T_{g_{1}}^{l} G_{B}\right), \\
\Omega^{1 / 2}\left(T_{g_{2}} G\right) & \simeq \Omega^{1 / 2}\left(T_{b_{R}(g)} B\right) \otimes \Omega^{1 / 2}\left(T_{g_{2}}^{r} G_{B}\right), \\
\Omega^{1 / 2}\left(T_{b_{L}(g)} B\right) & \simeq \Omega^{1 / 2}\left(T_{g}^{r} G_{A}\right), \\
\Omega^{1 / 2}\left(T_{b_{R}(g)} B\right) & \simeq \Omega^{1 / 2}\left(T_{g}^{l} G_{A}\right), \\
\Omega^{1 / 2}\left(T_{\left(g_{1}, g_{2}\right)} \delta(g)\right) & \simeq \Omega^{1 / 2}\left(T_{g_{1}}^{l} G_{B}\right), \\
\Omega^{1 / 2}\left(T_{\left(g_{1}, g_{2}\right)} \delta(g)\right) & \simeq \Omega^{1 / 2}\left(T_{g_{2}}^{r} G_{B}\right) .
\end{aligned}
$$

The first two isomorphisms follow from the fact that $b_{L}$ and $b_{R}$ are surjective submersions; the third and fourth are given, respectively, by $T_{b_{L}(g)} B \ni X \mapsto$ $X a_{R}(g) \in T_{g}^{r} G_{A}$ and $T_{b_{R}(g)} B \ni Y \mapsto a_{L}(g) Y \in T_{g}^{l} G_{A}$; the last two are due to the fact that $\pi_{1}: \delta(g) \ni\left(g_{1}, g_{2}\right) \mapsto g_{1} \in F_{B}^{l}(g)$ and $\pi_{2}: \delta(g) \ni\left(g_{1}, g_{2}\right) \mapsto$ $g_{2} \in F_{B}^{r}(g)$ are diffeomorphisms. Consequently,

$$
\begin{aligned}
& \Omega^{1 / 2}\left(T_{g_{1}} G\right) \simeq \Omega^{1 / 2}\left(T_{g}^{r} G_{A}\right) \otimes \Omega^{1 / 2}\left(T_{\left(g_{1}, g_{2}\right)} \delta(g)\right), \\
& \Omega^{1 / 2}\left(T_{g_{2}} G\right) \simeq \Omega^{1 / 2}\left(T_{g}^{l} G_{A}\right) \otimes \Omega^{1 / 2}\left(T_{\left(g_{1}, g_{2}\right)} \delta(g)\right) .
\end{aligned}
$$

In this way, having $\varphi\left(g_{1}\right), \psi\left(g_{2}\right)$, we can define a 1-density on $T_{\left(g_{1}, g_{2}\right)} \delta(g)$ with values in the one-dimensional vector space $\Omega^{1 / 2}\left(T_{g}^{l} G_{A}\right) \otimes \Omega^{1 / 2}\left(T_{g}^{r} G_{A}\right)$. Denote the resulting mapping by $\Phi_{g}$. The explicit formula for $\Phi_{g}(\varphi, \psi)$ is

$$
\left[\Phi_{g}(\varphi, \psi)(u)\right](v \wedge w)=\varphi\left(g_{1}\right)\left(w a \wedge \pi_{1}(u)\right) \psi\left(g_{2}\right)\left(\widetilde{a} v \wedge \pi_{2}(u)\right)
$$

where $u \in \Lambda^{\max } T_{\left(g_{1}, g_{2}\right)} \delta(g), v \in \Lambda^{\max } T_{g}^{l} G_{A}, w \in \Lambda^{\max } T_{g}^{r} G_{A}, a:=g^{-1} g_{1}$, $\widetilde{a}:=g_{2} g^{-1}$ and $\pi_{1}, \pi_{2}$ are defined above. Integrating $\Phi_{g}(\varphi, \psi)$ we get a bidensity on $G_{A}$, which will be denoted by $\Phi(\varphi, \psi)$. Finally, we define a mapping

$\widehat{\Phi}:(\varphi, \psi) \mapsto|Q|^{1 / 2} \Phi\left(\overline{s_{B}(\varphi)}, \psi\right)$, i.e. $\widehat{\Phi}(\varphi, \psi)(g)=|Q|^{1 / 2}(g) \int_{\delta(g)} \Phi_{g}\left(\overline{s_{B}(\varphi)}, \psi\right)$,

and prove the following 
Proposition 8.1. Let $\varphi, \psi$ be smooth, compactly supported half-densities on $G$. Then $\widehat{\Phi}(\varphi, \psi) \in \mathcal{A}\left(G_{A}\right), \kappa(\widehat{\Phi}(\varphi, \psi))=(\widehat{\Phi}(\psi, \varphi))^{*}$ and $\left(\eta_{\varphi \psi} \otimes \mathrm{id}\right) W=$ $\pi_{\text {id }}(\widehat{\Phi}(\varphi, \psi))$.

Proof. We begin by computing the mapping $\widehat{\Phi}$. Choose real, non-zero half-densities $\mu_{0}, \nu_{0}$ on $T_{e} B$ and $T_{e} A$, respectively, and let $\mu_{l}, \nu_{r}$ denote the corresponding left- and right-invariant half-densities on $B$ and $A$. Then $\nu_{r} \otimes \mu_{l}$ is a real, non-vanishing half-density on $A \times B$ and, since the mapping $A \times B \ni(a, b) \mapsto a b \in G$ is a diffeomorphism, this half-density defines a half-density on $G$ which will be denoted by $\varepsilon$. Explicitly,

$$
\varepsilon(g)(X g \wedge g Y)=\nu_{0}(X) \mu_{0}(Y) \quad \text { for } X \in \Lambda^{\max } T_{e} A, Y \in \Lambda^{\max } T_{e} B .
$$

Now we can write $\varphi=: f_{\varphi} \varepsilon, \psi=: f_{\psi} \varepsilon$ and $\widehat{\Phi}(\varphi, \psi)=: f_{\varphi \psi} \omega_{0}$. Define

$$
\alpha: A \ni a \mapsto \alpha(a):=\left(m_{B}\left(g, s_{B}(a g)\right), a g\right)=\left(g a_{R}(a g)^{-1}, a g\right) \in \delta(g) .
$$

This is clearly a diffeomorphism. Set $v:=g Y, w:=\tilde{Y} g, Y, \widetilde{Y} \in \Lambda^{\max } T_{e} B$, $\widetilde{u}:=X a, X \in \Lambda^{\max } T_{e} A$. Then

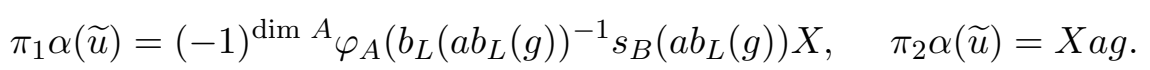

Inserting this into the formula for $\Phi_{g}$ and using (33) we get

$$
\begin{aligned}
& \left(\Phi_{g}\left(\overline{s_{B}(\varphi)}, \psi\right)(\alpha(X a))(g Y \wedge \tilde{Y} g)\right. \\
& =\left|\varphi_{A}\left(b_{L}\left(a b_{L}(g)\right)\right)\right|^{-1 / 2} \overline{s_{B}(\varphi)}\left(\tilde{Y} s_{B}\left(a b_{L}(g)\right) \wedge s_{B}\left(a b_{L}(g)\right) X\right) \psi(a g Y \wedge X a g) \\
& =\left|\frac{\psi_{B}(a)}{\psi_{A}\left(a_{R}\left(a b_{L}(g)\right)\right) \varphi_{A}\left(b_{L}\left(a b_{L}(g)\right)\right)}\right|^{1 / 2} \overline{\varphi\left(\widetilde{Y} a b_{L}(g) \wedge a b_{L}(g) X\right)} \psi(a g Y \wedge X a g),
\end{aligned}
$$

and since

$$
g X \wedge Y g=\frac{\psi_{A}\left(a_{R}(g)\right) \varphi_{A}\left(b_{L}(g)\right)}{\psi_{B}\left(a_{L}(g)\right) \varphi_{B}\left(b_{R}(g)\right)}(X g \wedge g Y)
$$

we obtain

$$
\begin{aligned}
& \Phi_{g}\left(\overline{s_{B}(\varphi)}, \psi\right)(\alpha(X a))(g Y \wedge \tilde{Y} g) \\
&=\left|\varphi_{B}\left(b_{L}(g)\right)\right|^{-1 / 2} \overline{\varphi\left(a b_{L}(g) \widetilde{Y} \wedge X a b_{L}(g)\right)} \psi(a g Y \wedge X a g) .
\end{aligned}
$$

Finally (we also use (10)),

$$
f_{\varphi \psi}(g)=\left|\frac{\psi_{B}\left(a_{R}(g)\right)}{\varphi_{B}\left(b_{R}(g)\right)}\right|^{1 / 2} \int_{A} \nu_{r}^{2}(a) \overline{f_{\varphi}\left(a b_{L}(g)\right)} f_{\psi}(a g) .
$$

From this equation it is clear that $f_{\varphi \psi} \omega_{0} \in \mathcal{A}\left(G_{A}\right)$. Having the above expression and using (25) one easily proves that $\kappa(\widehat{\Phi}(\varphi, \psi))=\widehat{\Phi}(\psi, \varphi)^{*}$. 
Now we are going to prove the last equality of the proposition. Clearly, it is enough to prove that

$$
(\varphi \otimes z \mid W(\psi \otimes t))=\left(z \mid \pi_{\mathrm{id}}(\widehat{\Phi}(\varphi, \psi)) t\right)
$$

for compactly supported, smooth half-densities $z, t$ on $G$. Let $\psi_{0}=: \varrho_{0} \otimes \nu_{l}$, where $\nu_{l}$ is the left-invariant half-density on $A$ defined by $\nu_{0}$ (the $\psi_{0}$ is as defined before the proof of Lemma 4.4). We can write $t=: f_{t} \psi_{0}$ and $z=: f_{z} \psi_{0}$.

Using (13) we have $\pi_{\text {id }}(\widehat{\Phi}(\varphi, \psi)) t=\left(f_{\varphi \psi} * f_{t}\right) \psi_{0}$ and

$$
\begin{aligned}
& \left(f_{\varphi \psi} * f_{t}\right)(g)=\int_{B} \mu_{l}^{2}(b) f_{\varphi \psi}\left(a_{L}(g) b\right) f_{t}\left(b_{L}\left(a_{L}(g) b\right)^{-1} g\right) \\
& =\int_{B} \mu_{l}^{2}(b)\left|\psi_{B}\left(a_{R}\left(a_{L}(g)^{-1} b\right)\right)\right| f_{\varphi \psi}\left(a_{L}(g) b_{L}\left(a_{L}(g)^{-1} b\right)\right) f_{t}\left(b^{-1} g\right) \\
& =\int_{B} \mu_{l}^{2}(b)\left|\psi_{B}\left(a_{R}\left(a_{L}(g)^{-1} b\right)\right)\right| f_{t}\left(b^{-1} g\right)\left|\frac{\psi_{B}\left(a_{R}\left(a_{L}(g) b_{L}\left(a_{L}(g)^{-1} b\right)\right)\right)}{\varphi_{B}\left(b_{L}\left(a_{L}(g)^{-1} b\right)\right)}\right|^{1 / 2} \\
& \quad \times \int_{A} \nu_{r}^{2}(a) \overline{f_{\varphi}(a b)} f_{\psi}\left(a a_{L}(g) b_{L}\left(a_{L}(g)^{-1} b\right)\right) \\
& =\int_{G} \varepsilon^{2}(\widetilde{g})\left|\frac{\psi_{B}\left(a_{R}\left(a_{L}(g)^{-1} b_{R}(\widetilde{g})\right)\right)}{\varphi_{B}\left(b_{L}\left(a_{L}(g)^{-1} b_{R}(\widetilde{g})\right)\right)}\right|^{1 / 2} \overline{\overline{f_{\varphi}(\widetilde{g})}} f_{\psi}\left(\widetilde{g} a_{L}\left(b_{R}(\widetilde{g})^{-1} g\right)\right) f_{t}\left(b_{R}(\widetilde{g})^{-1} g\right) \\
& =\int_{G} \varepsilon^{2}(\widetilde{g})\left|\frac{\psi_{B}\left(a_{R}\left(a_{L}(g)^{-1} b_{R}(\widetilde{g})\right)\right)}{\varphi_{B}\left(b_{L}\left(a_{L}(g)^{-1} b_{R}(\widetilde{g})\right)\right)}\right|^{1 / 2} \overline{\overline{f_{\varphi}(\widetilde{g})}\left(f_{\psi} \otimes f_{t}\right)\left(W^{-1}(\widetilde{g}, g)\right) .}
\end{aligned}
$$

In the second equality we use the diffeomorphism $B \ni b \mapsto b_{L}\left(a_{L}(g) b\right) \in B$, then the expression for $f_{\varphi \psi}$, and finally the definition of $\varepsilon(\widetilde{g}:=a b)$, and the formula (6) for $W^{-1}$.

On the other hand, computations similar to the ones in the proof of Lemma 6.5 yield $(\varphi \otimes z \mid W(\psi \otimes t))=(z \mid y)$ for $y=: f_{y} \psi_{0}$ and $f_{y}$ given by

$$
f_{y}(g)=\int_{G} \varepsilon^{2}(\widetilde{g}) \overline{f_{\varphi}(\widetilde{g})}\left[W\left(f_{\psi} \otimes f_{t}\right)\right](\widetilde{g}, g),
$$

where $W\left(f_{\psi} \otimes f_{t}\right)$ is defined by $W\left(f_{\psi} \varepsilon \otimes f_{t} \psi_{0}\right)=: W\left(f_{\psi} \otimes f_{t}\right)\left(\varepsilon \otimes \psi_{0}\right)$.

Since we can easily compare $\varepsilon \otimes \psi_{0}$ with $\psi_{0} \otimes \psi_{0}$, the expression for the function $W\left(f_{t} \otimes f_{\psi}\right)$ follows from the action of $W$ on $\psi_{0} \otimes \psi_{0}$ given in the proof of Lemma 4.4. In this way we obtain

$$
W\left(f_{\psi} \otimes f_{t}\right)(\widetilde{g}, g)=\left(f_{\psi} \otimes f_{t}\right)\left(W^{-1}(\widetilde{g}, g)\right)\left|\frac{\psi_{B}\left(a_{R}\left(a_{L}(g)^{-1} b_{R}(\widetilde{g})\right)\right)}{\varphi_{B}\left(b_{L}\left(a_{L}(g)^{-1} b_{R}(\widetilde{g})\right)\right)}\right|^{1 / 2} .
$$

Now insert this into the formula for $f_{y}$ and compare with the expression for $f_{\varphi \psi} * f_{t}$. This completes the proof of the proposition. 


\section{References}

[1] S. Baaj and G. Skandalis, Unitaires multiplicatifs et dualité pour les produits croisés de $C^{*}$-algèbres, Ann. Sci. École Norm. Sup. 26 (1993), 425-488.

[2] A. Connes, Noncommutative Geometry, Academic Press, San Diego, 1994.

[3] A. van Daele, Multiplier Hopf algebras, Trans. Amer. Math. Soc. 342 (1994), 917932 .

[4] M. Enock and J.-M. Schwartz, Kac Algebras and Duality of Locally Compact Groups, Springer, 1992.

[5] G. Kac, Extensions of groups to ring groups, Math. USSR-Sb. 5 (1968), 451-474.

[6] J. H. Lu and A. Weinstein, Poisson Lie groups, dressing transformations and Bruhat decomposition, J. Differential Geom. 31 (1990), 501-526.

[7] S. Majid, Physics for algebraists: Non-commutative and non-cocommutative Hopf algebras by a bicrossproduct construction, J. Algebra 130 (1990), 17-64.

[8] - , Hopf-von Neumann algebra bicrossproducts, Kac algebra bicrossproducts and classical Yang-Baxter equations, J. Funct. Anal. 95 (1991), 291-319.

[9] - Foundations of Quantum Group Theory, Cambridge Univ. Press, 1995.

[10] T. Masuda, Y. Nakagami and S. L. Woronowicz, $C^{*}$-algebraic framework for the duality of quantum groups, Int. J. Math. 14 (2003), 903-1001.

[11] J. Renault, Groupoid Approach to $C^{*}$-Algebras, Lecture Notes in Math. 793, Springer, 1980.

[12] P. Stachura, Manageability of multiplicative unitaries associated with double Lie groups, Lett. Math. Phys. 51 (2000), 135-143.

[13] -, Differential groupoids and $C^{*}$-algebras, math.QA/9905097; for a short exposition see also: $C^{*}$-algebra of a differential groupoid, Banach Center Publ. 51, Inst. Math., Polish Acad. Sci., 2000, 263-281.

[14] S. Vaes and J. Kustermans, Locally compact quantum groups, Ann. Sci. École Norm. Sup. 33 (2000), 837-934.

[15] S. Vaes and L. Vainerman, Extensions of locally compact quantum groups and the bicrossproduct construction, Adv. Math. 175 (2003), 1-101.

[16] S. L. Woronowicz, From multiplicative unitaries to quantum groups, Int. J. Math. 7 (1996), 127-149.

[17] S. Zakrzewski, Quantum and classical pseudogroups, Comm. Math. Phys. 134 (1990), 347-395.

[18] - Poisson Lie groups and pentagonal transformations, Lett. Math. Phys. 24 (1992), $13-19$.

Department of Mathematics

The George Washington University

Washington, DC 20052, U.S.A.
Department of Mathematical Methods in Physics University of Warsaw

Hoża 74

00-682 Warszawa, Poland E-mail: stachura@fuw.edu.pl

Received 15 June 2004;

in revised form 31 March 2005 